\title{
Subcoercive and Subelliptic Operators on Lie Groups: Variable Coefficients
}

\author{
By
}

A. F. M. ter ELST*t and Derek W. RoBINSON*

\begin{abstract}
Let $a_{1}, \cdots, a_{d^{\prime}}$ be an algebraic basis of rank $r$ in a Lie algebra $g$ of a connected Lie group $G$ and let $A_{2}$ be the left differential operator in the direction $a_{i}$ on the $L_{p}$-spaces with respect to the left, or right, Haar measure, where $p \in[1, \infty]$. We consider $m$-th order operators

$$
H=\sum c_{\alpha} A^{a}
$$

with complex variable bounded coefficients $c_{\alpha}$ which are subcoercive of step $r$, i.c., for all $g \in G$ the form obtained by fixing the $c_{a}$ at $g$ is subcoercive of step $r$ and the ellipticity constant is bounded from below uniformly by a positive constant. If the principal coefficients are $m$-times differentiable in $L_{\infty}$ in the directions of $a_{1}, \cdots, a_{d^{\prime}}$ we prove that the closure of $H$ generates a consistent interpolation semigroup $S$ which has a kernel. We show that $S$ is holomorphic on a non-empty $p$-independent sector and if $H$ is formally self-adjoint then the holomorphy angle is $\pi / 2$. We also derive 'Gaussian' type bounds for the kernel and its derivatives up to order $m-1$.
\end{abstract}

\section{$\S 1$. Introduction}

Several recent papers have been devoted to the development of a theory of second-order subelliptic differential operators with real coefficients acting on functions over a Lie group (see [1], [4], [3], [18], [19], [22], [23]). The subelliptic theory extends the theory of strongly elliptic operators described at length in [20] and the purpose of this paper is to further extend the theory in two different directions. First we generalize the theory to higher-order operators characterized by a condition of coercivity. Secondly, we allow complex-valued coefficients. The subsequent results then extend our earlier work, and that of Hebisch [13], [14], on subcoercive operators with constant coefficients to the setting of variable coefficients.

Communicated by M. Kashiwara, July 20, 1992.

AMS Subject Classification: 43A65, 22E45, 35B45, 35J30, 35J15, 35H05.

* Centre for Mathematics and its Applications, School of Mathematical Sciences, Australian National University, Canberra, ACT 0200, Australia.

+ Current address: Department of Mathematics and Comp. Sc., Eindhoven University of Technology, P.O. Box 513, 5600 MB Eindhoven, The Netherlands. 
In the sequel we adopt the general notation used in [20] and [10]. In particular, $G$ is a Lie group with left Haar measure $d g$ and right Haar measure $d \hat{g}$, but now the representation $U$ is identified with the left regular representation $L$ on the $L_{p}$-spaces, $p \in[1, \infty]$, with respect to the left, or right, Haar measure, $L_{p}=L_{p}(G ; d g)$ or $L_{\hat{p}}=L_{p}(G ; d \hat{g})$, respectively. We denote the norm on $L_{p}$ by $\|\cdot\|_{p}$ and the norm on $L_{\hat{p}}$ by $\left\|_{\|} \cdot\right\|_{\hat{p}}$. We assume throughout that $G$ is connected, but $G$ need not be unimodular. The modular function is denoted by $\Delta$. Let $a_{1}, \cdots, a_{d^{\prime}}$ be an algebraic basis for the Lie algebra $g$ of $G$, i. e., a finite sequence of linearly independent elements of $g$ which generate g. Thus there is an integer $r$ such that $a_{1}, \cdots, a_{d}$. together with all commutators $\left(\operatorname{ad} a_{\imath_{1}}\right) \cdots\left(\operatorname{ad} a_{\imath_{n-1}}\right)\left(a_{\imath_{n}}\right), i_{j}=1, \cdots, d^{\prime}$, where $n \leqq r$, span the vector space g. The smallest integer $r$ with this property is referred to as the rank of the subbasis and a vector space basis is defined to have rank one. Moreover, the algebraic basis determines in a canonical fashion (see, [20] Section IV.4c) a modulus function $g_{\mapsto} \mapsto g^{\prime}$ on the group. This function in turn determines a unique local dimension $D^{\prime}$ such that the ball $B_{\rho}^{\prime}=\left\{g \in G:|g|^{\prime}<\rho\right\}$ has Haar measure $\left|B_{\rho}^{\prime}\right|$ satisfying bounds $c_{1} \rho^{D^{\prime}} \leqq\left|B_{\rho}^{\prime}\right| \leqq c_{2} \rho^{D^{\prime}}$ for all $\rho \in\langle 0,1]$.

Next for all $i \in\left\{1, \cdots, d^{\prime}\right\}$ let $A_{\imath}$ be the infinitesimal generator of the one parameter group $t \mapsto L\left(\exp \left(t a_{\imath}\right)\right)$ from $\boldsymbol{R}$ into $L_{p}$ or $L_{\hat{p}}$. It will clear from the context on which space $A_{2}$ acts. We also denote by $A_{2} \varphi$ the pointwise left derivative in the direction $a_{\imath}$ of a function $\varphi: G \rightarrow C$. We use multi-index notation for products of the generators $A$. For $n \in \boldsymbol{N}$ let

$$
J_{n}\left(d^{\prime}\right)=\sum_{k=0}^{n}\left\{1, \cdots, d^{\prime}\right\}^{k}
$$

If $\alpha=\left(i_{1}, \cdots, i_{k}\right) \in\left\{1, \cdots, d^{\prime}\right\}^{k}$, we define $|\alpha|=k$ and $\lambda^{a}=A_{\imath_{1}} \cdots A_{\imath_{k}}$. Let $J\left(d^{\prime}\right)$ $=\cup_{n=1}^{\infty} J_{n}\left(d^{\prime}\right)$. Then for each $m \in \boldsymbol{N}$ we denote the subspace $\bigcap_{\alpha \in J_{m}\left(d^{\prime}\right)} D\left(A^{n}\right)$ in $L_{p}$ or $L_{\hat{p}}$, by $L_{p ; m}^{\prime}$ or $L_{\hat{p} ; m}^{\prime}$, respectively. We define a norm and a seminorm on $L_{p}^{\prime} ; m$ by setting

$$
\|\varphi\|_{p ; m}^{\prime}=\sup _{a \in J m\left(\alpha^{\prime}\right)}\left\|I^{n} \varphi\right\|_{p}, \quad N_{p ; m}^{\prime}(\varphi)=\sup _{|a|=m}\left\|A^{n} \varphi\right\|_{p},
$$

for each $\varphi \in L_{p ; m}^{\prime}$ and analogously we define $\|\cdot\|_{\hat{p} ; m}^{\prime}$ and $N_{\hat{p} ; m}^{\prime}$ on $L_{\hat{p} ; m}^{\prime}$. Let $L_{p ; \infty}=\bigcap_{m=1}^{\infty} L_{p ; m}^{\prime}$ and define $L_{\hat{p} ; \infty}$ similarly. We also adopt the corresponding notation $C_{0 ; m}^{\prime}$ for the subspaces $\bigcap_{\alpha \in J_{m}\left(d^{\prime}\right)} D\left(A^{a}\right)$ associated with the generators of left translations on $C_{0}=C_{0}(G)$.

An $m$-th order form is a function $C: J_{m}\left(d^{\prime}\right) \rightarrow C$ such that $C(\alpha) \neq 0$ for some $\alpha \in J_{m}\left(d^{\prime}\right)$ with $|\alpha|=m$. The principal part $P$ of $C$ is the form with $P(\alpha)=$ $C(\alpha)$ if $|\alpha|=m$ and $P(\alpha)=0$ if $|\alpha|<m$. The formal adjoint $C^{\dagger}$ of $C$ is the function $C^{\dagger}: J_{m}\left(d^{\prime}\right) \rightarrow C$ defined by $C^{\dagger}(\alpha)=(-1)^{|a|} \overline{C^{\prime}\left(\alpha_{*}\right)}$ where $\alpha_{*}=\left(i_{n}, \cdots, i_{1}\right)$ whenever $\alpha=\left(i_{1}, \cdots, i_{n}\right)$.

Next we want to introduce the concept of subcoercive form of step $s$, with $s \in \boldsymbol{N}$. Let $\mathrm{g}\left(d^{\prime}, s\right)$ denote the nilpotent Lie algebra with $d^{\prime}$ generators which 
is free of step $s$, i. e., the quotient of the free Lie algebra with $d^{\prime}$ generators $\tilde{a}_{1}, \cdots, \tilde{a}_{d}$. by the ideal generated by the commutators of order at least $s+1$. Further let $\tilde{G}=G\left(d^{\prime}, s\right)$ be the connected simply connected Lie group with Lie algebra $g\left(d^{\prime}, s\right)$. Let $A_{\imath}$ be the infinitesimal generator of left translations $L_{\widetilde{G}}$ in $L_{2}(\tilde{G} ; d g)$ corresponding to $\tilde{a}_{\imath}$. We deline

$$
\partial L_{\widetilde{G}}(C)=\sum_{n \in J m\left(d^{\prime}\right)} C(\alpha) A^{a}
$$

as the operator in $L_{2}(\tilde{G} ; d g)$ with domain $L_{2 ; \infty}(G ; d g)$. We say that $C$ is an $m$-th order subcoercive form of step $s$ if $m$ is even and there exists $\mu>0$ such that

$$
\operatorname{Re}\left(\partial L_{\widetilde{G}}(P) \varphi, \varphi\right) \geqq \mu\left(N_{2 ; m / 2}^{\prime}(\varphi)\right)^{2}
$$

for all $\varphi \in L_{2 ; \infty}(\grave{G} ; d g)$. The largest such $\mu$ is called the ellipticity constant of $C$.

Now we introduce some new definitions. We want to study operators with variable coefficients, so we have to introduce forms which depend on the points of $G$. Let $m \in N$ be even and let $C: J_{m}\left(d^{\prime}\right) \times G \rightarrow C$. For all $\alpha \in J_{m}\left(d^{\prime}\right)$ define $c_{\alpha}: G \rightarrow C$ by $c_{\alpha}(g)=C(\alpha, g)$ and for $g \in G$ define $C_{g}: J_{m}\left(d^{\prime}\right) \rightarrow C$ by $C_{g}(\alpha)=$ $C(\alpha, g)$. We refer to the $c_{\alpha}$ as the coefficients of $C$. Now we call $C$ an $m$-th order form if $c_{\alpha} \in L_{\infty}$ for all $\alpha \in J_{m}\left(d^{\prime}\right)$ and, moreover, for all $g \in G$ there exists $\alpha \in J_{m}\left(d^{\prime}\right)$ such that $C(\alpha, g) \neq 0$ and $|\alpha|=m$. If $c_{\alpha} \in L_{\infty ;|\alpha|}^{\prime}$ for all $\alpha \in J_{m}\left(d^{\prime}\right)$ we define the formal adjoint $C^{\dagger}$ of $C$ by

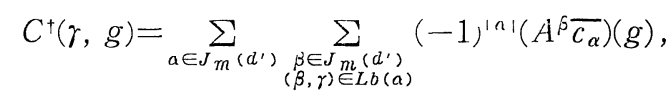

where $L b(\alpha)$ is the set of all $(\beta, \gamma) \in J_{m}\left(d^{\prime}\right)^{2}$ such that $\beta$ is a multi-index obtained from $\alpha$ by omission of some indices and $\gamma$ is the multi-index formed by the omitted indices, i.e., the $(\beta, \gamma)$ occurring are the pairs of multi-indices in the Leibniz formula for the multi-derivative.$^{a}$ of a product. If $s \in \boldsymbol{N}$ we call $C$ a subcoercive form of step $s$ if there exists $\mu>0$ such that for all $g \in G$ the form $C_{g}$ is a subcoercive form of step $s$ and the ellipticity constant for $C_{g}$ is larger than or equal to $\mu$. The maximum of all possible $\mu$ is called the ellipticity constant for $C$. For $g \in G$ let $P_{g}$ be the principal part of $C_{g}$ and with $\tilde{G}=G\left(d^{\prime}, s\right)$ let

$$
\nu=\sup \left\{\left|\operatorname{Im}\left(\partial L_{\widetilde{G}}\left(P_{g}\right) \varphi, \varphi\right)\right| /\left(N_{2 ; m / 2}^{\prime}(\varphi)\right)^{2}: \varphi \in L_{2 ; \infty}(\tilde{G} ; d h), \varphi \pm 0, g \in G\right\} .
$$

Then set $\theta_{C}=\arctan \left(\mu \nu^{-1}\right) \in\langle 0, \pi / 2]$.

If the form $C$ is subcoercive of step $r$, where $r$ is the rank of the algebraic basis $a_{1}, \cdots, a_{d^{\prime}}$, we study the affiliated subcoercive operator

$$
H=d L(C)=\sum_{a \in J m\left(d^{\prime}\right)} c_{\alpha} 4^{a}
$$

on the $L_{p^{-}}$, or $L_{\hat{p}^{-}}$, spaces, with domain $D(H)=L_{p ; m}^{\prime}$ or $D(H)=L_{\hat{p} ; m}^{\prime}$, respec- 
tively. If the formal adjoint $C^{\dagger}$ is defined we also define $H^{\dagger}=d L\left(C^{\dagger}\right)$ to be the subcoercive operator affiliated with $C^{\dagger}$. The main result of this paper is that if $c_{\alpha} \in L_{p ; m}^{\prime}$ for all $\alpha$ with $|\alpha|=m$ then the closure $\bar{H}$ of $H$ generates a continuous semigroup $S$ which interpolates consistently between the $L_{p^{-}}$, and $L_{\hat{p}^{-}}$, spaces and which is holomorphic on each of these spaces in a sector with angle larger or equal to $\theta_{C}$. Moreover, the action of $S$ is determined by an integral kernel which satisfies appropriate Gaussian bounds and the smoothness properties typical of elliptic regularity.

In the important case $m=2$ our results cover operators of the form

$$
H=-\sum_{i, j=1}^{d^{\prime}} c_{i j} A_{i} A_{j}+\sum_{i=1}^{d^{\prime}} c_{i} A_{i}+c_{0}
$$

with $c_{i j} \in L_{\infty ; 2}^{\prime}, c_{i}, c_{0} \in L_{\infty}$ and with the real part of the matrix $C=\left(c_{i j}\right)$ uniformly strictly positive-definite, i. e., there is a $\mu>0$, the ellipticity constant, such that $2^{-1}\left(C+C^{*}\right) \geqq \mu I$, in the sense of matrices, uniformly over the group. Then the corresponding semigroup $S$ has a kernel $K$ satisfying bounds

$$
\left|K_{t}(g ; h)\right| \leqq a t^{-D^{\prime} / 2} e^{\omega t} e^{-b\left(1 g h-1_{1}^{\prime}\right) 2^{2}-1}
$$

for all $g, h \in G, t>0$. Moreover, if $\nu I \geqq(2 i)^{-1}\left(C-C^{*}\right) \geqq-\nu I$ then $S$, on each of the $L_{p^{-}}$, and $L_{\hat{p}^{-}}$, spaces, is holomorphic in a sector with angle larger or equal to $\arctan \left(\mu \nu^{-1}\right)$. In particular, if the matrix of principal coefficients is hermitian then $S$ is holomorphic in the open right half-plane.

\section{§. Smooth Coefficients}

In this section we consider $m$-th order subcoercive operators $H=d L(C)$, associated with an $m$-th order subcoercive form $C$ of step $r$ and coefficients $c_{\alpha} \in C_{b ; \infty}$, acting on the $L_{p^{-}}$, and $L_{\hat{p}^{-}}$, spaces and examine the heat semigroups generated by their closures. We prove the existence of these semigroups and establish that each such semigroup is determined by an integral kernel. By this we mean that for each $t>0$ there exists a function $K_{t} \in C_{b}(G \times G)$ which is rapidly decreasing as $\left|g h^{-1}\right|^{\prime} \rightarrow \infty$ and

$$
\left(S_{t} \varphi\right)(g)=\int_{G} d \hat{g} K_{t}(g ; h) \varphi(h)
$$

for all $\varphi \in L_{p}$ or $\varphi \in L_{\hat{p}}$. Our approach is to first construct a suitable kernel $t \mapsto K_{t}$ as a function over $G \times G$ by a parametrix expansion and then use it to prove the existence of the semigroup. In the course of the analysis we obtain pointwise bounds on $K_{t}$ and its left derivatives. These bounds are largely independent of the smoothness of the $c_{\alpha}$; they only involve the $m$-th order subelliptic left derivatives of the principal coefficients. Subsequently these bounds can be used to extend the semigroup results to operators with weaker smoothness of the coefficients and in particular to operators for which the only 
smoothness hypotheses are on the principal coefficients.

Let $K_{t}(g ; h)$ denote the values of $K$. The kernel should satisfy the generalized heat equation

$$
\left(\left(\partial_{t}+H\right) K_{t}\right)(g ; h)=\boldsymbol{\delta}(t) \boldsymbol{\delta}\left(g h^{-1}\right)
$$

on $\boldsymbol{R} \times G \times G$ with measure $d t \times d g \times d \hat{h}$, where $\partial_{t}=\partial / \partial t$, the differential operator $H$ acts on the variable $g$ and $\delta$ denotes both the point measure at the origin of $\boldsymbol{R}$ and at the identity of $G$. Moreover, one should have $K_{t}(g ; h)=0$ for $t<0$. Now we aim to construct $K$ with these properties by successive approximations. Let $P$ be the principal part of the form $C$. The zero order approximation $K^{(0)}$ of $K$ is defined in terms of the kernel $K^{g}$ of the semigroup $S^{g}$ generated by the closure of the principal part $H_{g}^{P}=d L\left(P_{g}\right)$ of the subcoercive operator $H_{g}=d L\left(C_{g}\right)$ obtained by fixing the coefficients $c_{\alpha}$ of $H$ with their values at the point $g$, i. e.,

$$
H_{g}=\sum_{\alpha \in J_{m}\left(d^{\prime}\right)} c_{\alpha}(g) A^{n}
$$

and the corresponding principal part is given by

$$
H_{g}^{P}=\sum_{\alpha ;|a|=m} c_{\alpha}(g) A^{\alpha}
$$

(see [10] Theorem 5.4 and Section 7). Thus $(t, h) \mapsto K_{t}^{g}(h)$ is a distribution over $\boldsymbol{R} \times G$, with measure $d t \times d \hat{h}$, satisfying the differential equation

$$
\left(\left(\partial_{t}+H_{g}^{P}\right) K_{t}^{g}\right)(h)=\delta(t) \delta(h) .
$$

In addition one has $K_{t}^{g}=0$ for $t<0$ and $K_{t}^{g} \in C_{0 ; \infty}(G)$ for $t>0$. Further we define $K_{t}^{g}=0$ if $t=0$.

Now the zero-order approximation $K^{(0)}$ of $K$ is introduced by setting

$$
K_{t}^{(0)}(g ; h)=K_{t}^{g}\left(g h^{-1}\right) \text {. }
$$

Formally it then follows that as distribution on $R \times G \times G$, with measure $d t \times$ $d g \times d \hat{h}$,

$$
\left(\left(\partial_{t}+H\right) K_{t}^{(0)}\right)(g ; h)=\delta(t) \delta\left(g h^{-1}\right)+L_{t}(g ; h)
$$

where $L_{t}$ is given by

$$
L_{t}(g ; h)=\sum_{\alpha \in J_{m}\left(d^{\prime}\right)} \sum_{\substack{(\beta, r) \in b b(\alpha) \\|\beta| \neq m}} c_{\alpha}(g)\left(A^{\beta} \partial^{r} K_{t}^{g}\right)\left(g h^{-1}\right)
$$

with $A$ the left derivatives of $K_{t}^{g}$ and $\partial$ the left derivatives with respect to the exponent variable. Therefore the solution of (1) is formally expressed by the expansion

$$
K_{t}=\sum_{n \geq 0} K_{t}^{(n)}
$$

with the $K^{(n)}$ defined recursively by 


$$
K_{t}^{(n)}=-\left(K^{(n-1)} \hat{*} L\right)_{\iota}
$$

and the convolution product $\hat{*}$ given by

$$
(\varphi \hat{*} \psi)_{t}(g ; h)=\int_{R} d s \int_{G} d \hat{k} \varphi_{t-s}(g ; k) \psi_{s}(k ; h) .
$$

Note that $K_{t}^{(0)}=0=L_{t}$ for $t \leqq 0$. Thus one automatically has $K_{t}^{(n)}=0$ for $t \leqq 0$. Now using the estimates and arguments of [4] and [10] we will establish that $K$ is well defined by this series expansion and satisfies 'Gaussian' bounds. This is then used to prove that the closure of $H$ generates a continuous semigroup $S$ with $K$ as kernel.

Theorem 2.1. Let $C$ be an $m$-th order subcoercive form of step $r$ with coefficients $c_{\alpha} \in C_{b ; \infty}, H=d L(C)$ and $H^{\dagger}=d L\left(C^{\dagger}\right)$ the operator associated with the formal adjoint on the dual space.

I. $H$ is closable on each of the $L_{p^{-}}$, and $L_{\hat{p}^{-}}$, spaces, $\bar{H}=\left(H^{\dagger}\right)^{*}$, and the closures $\bar{H}$ generate a consistent interpolating semigroup $S$.

II. $S$ is holomorphic in a sector $\Delta_{\theta}=\{z \in C:|\arg z|<\theta\}$ with $\theta \geqq \theta_{C}$.

III. The action of $S_{t}$ is determined by an integral kernel $K_{t} \in C_{b}(G \times G)$ and $t \rightarrow K_{t}(g ; h)$ is analytic from $\langle 0, \infty\rangle$ into $C$ for all $g, h \in G$.

IV. The closure of the formal adjoint $H^{+}$of $H$ generates a consistent interpolating semigroup $S^{\dagger}$ dual to $S$ with a kernel $K^{\dagger}$ such that

$$
K_{t}^{\dagger}(g ; h)=\Delta(g)^{-1} \Delta(h) \overline{K_{t}(h ; g)}
$$

for all $g, h \in G$ and $t \in \boldsymbol{R}$.

V. For each $n \in \boldsymbol{N}$ the derivative $\partial_{t}^{n} K_{t}$, with $t>0$ fixed, is $(m-1)$-times $L_{\infty}$ (left-)differentiable in both variables and the derivatives with respect to one variable are $(m-1)$ times differentiable with respect to the other.

VI. There exist $a, b, c>0$ and $\omega \geqq 0$ such that

$$
\left|\left(A^{\alpha} \partial_{t}^{n} K_{t}\right)(g ; h)\right| \leqq a c^{n} n ! t^{-n} t^{-\left(D^{\prime}+|\alpha|\right) / m} e^{\omega t} e^{-b\left(\left(\left.1 g h^{-1}\right|^{\prime}\right) m_{t-1}\right)^{1 /(m-1)}}
$$

for all $n \in \boldsymbol{N}, t>0, g, h \in G$ and all multi-indices $\alpha$ with $|\alpha|<m$ where the $A$ are the left derivatives with respect to the first variable. The values of $a, b, c$ and $\omega$ depend on the coefficients $c_{\alpha}$ through the ellipticity constant $\mu$ and the parameter

$$
\|C\|_{m}^{\prime}=\sum_{\alpha ;|\alpha|=m}\left\|c_{a}\right\|_{\infty ; m}^{\prime}+\sum_{\alpha \in J} \sum_{m-1\left(d^{\prime}\right)}\left\|c_{\alpha}\right\|_{\infty} .
$$

VII. For each $p \in[1, \infty], t>0$ one has $S_{t} L_{p} \subseteq L_{p ; m-1}^{\prime}, S_{t} L_{\hat{p}} \subseteq L_{\hat{p} ; m-1}^{\prime}$, there exist $a>0$ and $\omega \geqq 0$, depending on the coefficients $c_{\alpha}$ through $\mu$ and $\|C\|_{m}^{\prime}$, such that

$$
\left\|A^{\alpha} S_{t}\right\|_{p \rightarrow p} \leqq a t^{-|a| / m} e^{\omega t}, \quad\left\|A^{a} S_{t}\right\|_{\hat{p} \rightarrow \hat{p}} \leqq a t^{-|\alpha| / m} e^{\omega t}
$$

and $A^{\alpha} K_{t}$ is the kernel of $A^{\alpha} S_{t}$, for all $\alpha \in J_{m-1}\left(d^{\prime}\right)$. 
Proof. Since $H$ is densely defined and $H^{\dagger}$ is densely defined on the dual space it follows that $H$ is closable. The rest of the proof is now based on analysis of the parametrix expansion defined formally by (4) and (5) following the general reasoning of [4] and [10] combined with various general techniques of semigroup theory.

The first aim is to prove that the recursion relation (5) is well defined and that the series (4) is convergent on the weighted spaces $\mathcal{L}_{\infty}^{o}, \rho \geqq 0$, consisting of the measurable functions $\varphi$ over $G \times G$ for which the norm

$$
\|\varphi\|_{\infty}^{\rho}=\underset{g, h \in G}{\operatorname{ess} \sup } e^{\rho\left|g h^{-1}\right|^{\prime}}|\varphi(g ; h)|
$$

is finite. This requires estimates on the associated $L_{1}$-spaces, $\mathcal{L}_{1}^{\rho}$ and $\mathcal{L}_{1}^{o}$, with norms

$$
\|\phi\|_{1}^{\rho}=\underset{g \in G}{\operatorname{ess} \sup } \int_{G^{\prime}} d \hat{h} e^{\rho\left|g h^{-1}\right|^{\prime}}|\phi(g ; h)|
$$

and

$$
\|\chi\|_{1}^{\rho}=\underset{h \in G}{\operatorname{ess} \sup } \int_{G} d \hat{g} e^{\rho\left|g h^{-1}\right|^{\prime}}|\chi(g ; h)|
$$

respectively. The recursion relations (5) then formally give the coupled integral inequalities

$$
\left\|K_{t}^{-(l)}\right\|_{\infty}^{\rho} \leqq \int_{0}^{t} d s\left(\left\|K_{t-s}^{(n-1)}\right\|_{\infty}^{\rho}\left\|L_{s}\right\|_{\stackrel{1}{\rho}}^{\rho}\right) \wedge\left(\mid\left\|K_{t-s}^{(n-1)}\right\|_{1}^{\rho}\left\|L_{s}\right\|_{\infty}^{\rho}\right)
$$

and

$$
\left\|K_{t}^{(n)}\right\|_{1}^{\rho} \leqq \int_{0}^{t} d s\left\|K_{t-s}^{(n-1)}\right\|_{1}^{\rho}\left\|L_{s}\right\|_{1}^{\rho} .
$$

Therefore to bound the $K^{(n)}$ one needs suitable bounds on $\left\|K_{t}^{(0)}\right\|_{1}{ }_{1}$. $\left\|K_{t}^{(0)}\right\|_{\infty}^{\infty}$, $\left\|L_{l}\right\|_{1}^{o},\left\|L_{l}\right\|_{\infty}^{\rho}$ and $\left\|L_{l}\right\|_{1}^{\rho}$. It will then follow from these bounds that the recursion relation (5) is well defined and that the coupled integral inequalities (6) and (7) can be solved.

The necessary bounds on $K_{t}^{(0)}$ and $L_{t}$ follow from the pointwise Gaussian bounds on $K_{t}^{g}(h)$ and its derivatives with respect to $g$ and $h$. The bounds on the derivatives with respect to $h$ are obtained in [10] Theorem 7.1.IV. Explicitly, for all $\alpha \in J\left(d^{\prime}\right)$ there exist $a, b, \omega>0$ such that

$$
\left|\left(A^{a} K^{a} K_{t}^{g}\right)(h)\right| \leqq a t^{-\left(D^{\prime}+|a|\right) / m} e^{\omega t} e^{-b\left(\left(|h|^{\prime}\right) m t^{-1,1 /(i n-1)}\right.}
$$

for all $h \in G$ and $t>0$. But since the constants $a, b$ and $\omega$ depend continuously on the ellipticity constant for the form $C_{g}$ and on the $C_{g}(\beta)$ with $|\beta|=m$, we may assume that the estimates (8) are uniform in $g$. Note that $A^{a} K_{t}^{g} \in L_{1} \cap C_{0}$, so convolution products of these kinds of functions are elements of $L_{1} \cap C_{0}$. We also need estimates for derivatives of $-1^{a} K_{t}^{g}$ with respect to $g$. For $t>0$ and $k \in \boldsymbol{N}$ let 


$$
\Omega_{k}(t)=\left\{\left(u_{1}, \cdots, u_{k}\right) \in\langle 0, \infty\rangle^{k}: u_{1}+\cdots+u_{k}=t\right\}
$$

and let $d \Omega$ denote Lebesgue measure on this surface.

Lemma 2.2. Let $P$ be a pure $m$-th order subcoercive form of step $r$ with coefficients $c_{\alpha} \in C_{b}(G)$ and let $H^{P}=d L(P)$. Further let $\alpha_{1} \in J_{m-1}\left(d^{\prime}\right), k \in\{2, \cdots$, $m+1\}$ and $\alpha_{2}, \cdots, \alpha_{k} \in J_{m}\left(d^{\prime}\right)$ with the constraint $\left|\alpha_{1}\right|+k-1 \leqq m$. For $g \in G$ let $K_{t}^{g}$ be the kernel of the semigroup $S_{t}^{g}$ generated by the closure of the subcoercive operator $H_{g}^{P}=d L\left(P_{g}\right)$ associated with the subcoercive form $P_{g}$.

I. There exist $a, b, \omega>0$ such that for all $h \in G$, all $t>0$ and all $g_{1}, \cdots$, $g_{k} \in G$

$$
\begin{aligned}
\int_{\Omega_{k}(t)} d \Omega\left(u_{1}, \cdots,\right. & \left.u_{k}\right)\left|\left(A^{\alpha_{1}} K_{u_{1}}^{g_{1} * \cdots * A^{a}{ }_{k}} K_{u_{k}}^{g_{k}}\right)(h)\right| \\
& \leqq a t^{-\left(D^{\prime}+\left|\alpha_{1}\right|\right) / m} e^{\omega t} e^{-b\left(\left(|h|^{\prime}\right) m_{t-1) 1 /(m-1)}\right.} .
\end{aligned}
$$

II. Fix $g \in G$ and $t>0$. For $\beta \in J_{\left|\alpha_{1}\right|}\left(d^{\prime}\right)$ define $F_{\beta}: G \rightarrow C$ by

$$
F_{\beta}(h)=\int_{\Omega_{k}(t)} d \Omega\left(u_{1}, \cdots u_{k}\right)\left(A^{\beta} K_{u_{1}}^{g} * A^{a_{2}} K_{u_{2}}^{g} * \cdots * A^{a_{k}} K_{u_{k}}^{g}\right)(h) .
$$

Then $F_{\beta} \in C_{0 ;\left|\alpha_{1}\right|-|\beta|}^{\prime}$ and $A^{\gamma} F_{\beta}=F_{\langle\gamma, \beta\rangle}$ for all $\gamma \in J_{: \alpha_{1}|-| \beta \mid}\left(d^{\prime}\right)$.

III.

$$
\begin{array}{r}
\lim _{g_{1}, \cdots, g_{k} \rightarrow g} \int_{\Omega_{k}(t)} d \Omega\left(u_{1}, \cdots, u_{k}\right)\left(4^{a_{1}} K_{u_{1}}^{g} g_{1} \cdots * A^{\alpha_{k}} K_{u_{k}}^{g}\right)(h) \\
\quad=\int_{\Omega_{k}(t)} d \Omega\left(u_{1}, \cdots, u_{k}\right)\left(A^{\alpha_{1}} K_{u_{1}}^{g} * \cdots * A^{a_{k}} K_{u_{k}}^{g}\right)(h)
\end{array}
$$

for all $g \in G$ uniformly for $h \in G$.

IV. The function $(g, h) \mapsto K_{t}^{g}(h)$ is continuous from $G \times G$ into $C$ for all $t>0$.

Remark. The main difficulty in handling the integrals in the lemma arises because one can have $\left|\alpha_{i}\right|=m$ for any $i \in\{2, \cdots, k\}$. Then the integral over $u_{\imath}$ has a singularity $u_{i}^{-D^{\prime} / m-1}$. The contribution $u_{\imath}^{-D^{\prime} / m}$ is effectively cancelled by the convolution integral but the $u_{i}^{-1}$ remains and is non-integrable at $u_{\imath}=0$. This problem is exacerbated if one has several multi-indices with $\left|\alpha_{\imath}\right|=m$. But these difficulties can be circumvented by integrating by parts and effectively passing the derivatives to the left. This is the basic technique that we use in the proof. Note, however, that a simple counting argument indicates that this technique is only applicable under the condition $\left|\alpha_{1}\right|+k-1 \leqq m$. Non-integrable singularities are unavoidable if this condition is violated.

Proof. First, let $u_{1}, u_{2}, b>0$ and let $\varphi$ and $\phi$ be measurable functions which satisfy bounds

$$
\begin{aligned}
& |\varphi(g)| \leqq u_{1}^{-D^{\prime} / m} e^{-b\left(\left(|g|^{\prime}\right) m_{u}^{-1}\right)^{1 /(m-1)}} \\
& |\phi(g)| \leqq u_{2}^{-D^{\prime} / m} e^{-b\left(\left(|g|^{\prime}\right) m_{2}^{-1}\right)^{1 /(m-1)}}
\end{aligned}
$$


for all $g \in G$. Then we argue that there exist $c, \omega>0$, depending only on $b$, such that

$$
|(\varphi * \phi)(g)| \leqq c\left(u_{1}+u_{2}\right)^{-D^{\prime} / m} e^{-b^{\prime}\left(\left(1 g l^{\prime}\right)^{m}\left(u_{1}+u_{2}\right)^{-1}\right) 1 /(m-1)} e^{\omega\left(u_{1}+u_{2}\right)}
$$

for all $g \in G$, where $b^{\prime}=2^{-1-m /(m-1)} b$.

The proof is based on the observation that for all $b>0$ there exist $c, \omega>0$, depending only on $b$ such that

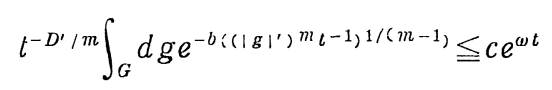

for all $t>0$.

There are two cases to consider. First, assume that $u_{1} \leqq u_{2}$. Then

$$
(\varphi * \phi)(g)=\int_{G} d h \varphi(h) \phi\left(h^{-1} g\right) .
$$

But $|g|^{\prime} \leqq|h|^{\prime}+\left|h^{-1} g\right|^{\prime}$ and therefore

$$
\left(\left|h^{-1} g\right|^{\prime}\right)^{m /(m-1)} \geqq 2^{-m /(m-1)}\left(|g|^{\prime}\right)^{m /(m-1)}-\left(|h|^{\prime}\right)^{m /(m-1)} .
$$

Hence

$$
\begin{aligned}
& \left(\left(|h|^{\prime}\right)^{m} u_{1}^{-1}\right)^{1 /(m-1)}+\left(\left(\left|h^{-1} g\right|^{\prime}\right)^{m} u_{2}^{-1}\right)^{1 /(m-1)} \\
& \quad \geqq 2^{-m /(m-1)}\left(\left(|g|^{\prime}\right)^{m} u_{2}^{-1}\right)^{1 /(m-1)}+\left(|h|^{\prime}\right)^{m /(m-1)}\left(u_{1}^{-1 /(m-1)}-u_{2}^{-1 /(m-1)}\right) \\
& \geqq 2^{-m /(m-1)}\left(\left(|g|^{\prime}\right)^{m}\left(u_{1}+u_{2}\right)^{-1}\right)^{1 /(m-1)} .
\end{aligned}
$$

Consequently

$$
\begin{aligned}
|(\varphi * \psi)(g)| & \leqq u_{1}^{-D^{\prime} / m} u_{2}^{-D^{\prime} / m} e^{-b^{\prime}\left(\left(|g|^{\prime}\right) m\left(u_{1}+u_{2}\right)^{-1}\right) 1 /(m-1)} \int_{G} d h e^{-2^{-1} b\left(\left(|h|^{\prime}\right) m_{u_{1}}^{-1}\right)^{1 /(m-1)}} \\
& \leqq c u_{2}^{-D^{\prime} / m} e^{-b^{\prime}\left(\left(|g|^{\prime}\right)^{m}\left(u_{1}+u_{2}\right)^{-1}\right)^{1 /(m-1)}} e^{\omega u_{1}} \\
& \leqq 2^{D^{\prime} / m} c\left(u_{1}+u_{2}\right)^{-D^{\prime} / m} e^{-b^{\prime}\left(\left(\left.! g\right|^{\prime}\right)^{m}\left(u_{1}+u_{2}\right)^{-1}\right)^{1 /(m-1)}} e^{\omega\left(u_{1}+u_{2}\right)}
\end{aligned}
$$

where $c$ and $\omega$ depend only on $b$.

Secondly, suppose that $u_{2} \leqq u_{1}$ and remark that

$$
(\varphi * \phi)(g)=\int_{G} d h \varphi(g h) \psi\left(h^{-1}\right) .
$$

Using

$$
\left(|g h|^{\prime}\right)^{m /(m-1)} \geqq 2^{-m /(m-1)}\left(|g|^{\prime}\right)^{m /(m-1)}-\left(|h|^{\prime}\right)^{m /(m-1)}
$$

one then obtains

$$
\left(\left(|g h|^{\prime}\right)^{m} u_{1}^{-1}\right)^{1 /(m-1)}+\left(\left(|h|^{\prime}\right)^{m} u_{2}^{-1}\right)^{1 /(m-1)} \geqq 2^{-m /(m-1)}\left(\left(|g|^{\prime}\right)^{m}\left(u_{1}+u_{2}\right)^{-1}\right)^{1 /(m-1)}
$$

and estimate (9) follows by a similar calculation.

Now we turn to the estimation of the first integral in the lemma. We begin by fixing $a, b, \omega>0$ such that for all $\alpha \in J_{m}\left(d^{\prime}\right)$ 


$$
\begin{aligned}
& \left|\left(A^{\prime \prime} K_{t}^{g}\right)(h)\right| \leqq a t^{-\left(D^{\prime}+|a|\right) / m} e^{\omega t} e^{-b\left(\left(|h|^{\prime}\right)^{m} t-1,1 /(m-1)\right.} \\
& \left|\left(A^{a} K_{t}^{g, \dagger}\right)(h)\right| \leqq a t^{-\left(D^{\prime}+|a|\right) / m} e^{(\omega t} e^{-b\left(\left(|h|^{\prime}\right)\right)^{m} t-1,1 /(m-1)}
\end{aligned}
$$

for all $t>0$ and $g \in G$. Here $K_{t}^{g, \tau}$ is the kernel of the semigroup $S_{t}^{g, T}$ generated by the closure of the subcoercive operator $d L\left(\left(P_{g}\right)^{*}\right)$ associated with the form $\left(P_{g}\right)^{\dagger}$. Note that the constants $a, b$ and $\omega$ depend only on the $\left\|c_{\alpha}\right\|_{\infty}$, with $|\alpha|$ $=m$ and the ellipticity constant $\mu$, in particular this is the case for $K_{t}^{g, 1}$.

Next we use the equality

$$
\Omega_{k}(t)=\bigcup_{j=1}^{k}\left\{\left(u_{1}, \cdots, u_{k}\right) \in\langle 0, t\rangle^{k}: u_{1}+\cdots+u_{r}=t, u_{\jmath} \geqq k^{-1} t\right\}
$$

to estimate the integral.

Let $t>0$ and $g_{1}, \cdots, g_{k} \in G$. Further let $j \in\{1, \cdots, k\}$ and $u_{1}, \cdots, u_{k} \in\langle 0, t\rangle$. Suppose that $u_{\jmath} \geqq k^{-1} t$ and $u_{1}+\cdots+u_{k}=l$. For all $l \Subset\{2, \cdots, k\}$ let $\alpha_{l}^{\prime}, \alpha_{l}^{\prime \prime} \in J_{m}\left(d^{\prime}\right)$ be such that $\alpha_{l}=\left\langle\alpha_{l}^{\prime}, \alpha_{l}^{\prime \prime}\right\rangle$ and

$$
\left.\begin{array}{l}
\left|\alpha_{l}^{\prime}\right|=\left(m-\left|\alpha_{1}\right|-l+1\right) \wedge\left|\alpha_{l}\right| \\
\left|\alpha_{l}^{\prime \prime}\right|=\left(\left|\alpha_{l}\right|-m+\left|\alpha_{1}\right|+l-1\right) \vee 0
\end{array}\right\} \text { if } l<j+1,
$$

Furthermore define the operation $\varphi \mapsto \varphi^{2}$ on functions over $G$ by

$$
\varphi^{\sim}(g)=\Delta(g)^{-1} \overline{\varphi\left(g^{-1}\right)}
$$

for all $g \in G$. Then

$$
\begin{aligned}
& A^{a_{1}} K_{u_{1}}^{g_{1}} \cdots * \mathcal{H}^{a_{k}} K_{u_{k}}^{g} \\
& =A^{\alpha_{1}} S_{u_{1}}^{g_{1}}-\mathcal{1}^{a_{2}} S_{u_{2}}^{g_{2}} \cdots A^{\alpha_{k}} S_{u_{k} / 2}^{g} K_{u_{k} / 2}^{k}
\end{aligned}
$$

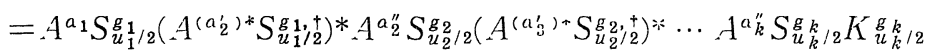

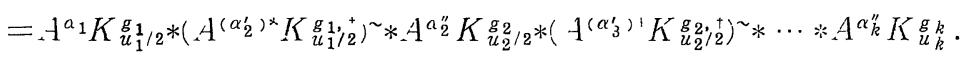

So by the previous observation, there exist $c, b^{\prime}, \omega^{\prime}>0$, depending only on $b$ and $\omega$, such that

$$
\begin{aligned}
& \left|\left(A^{a_{1}} K_{u_{1}}^{g_{1} * \cdots * A^{a_{k}}} K_{u_{k}}^{g}\right)(h)\right| \\
& \leqq \\
& a^{2 k-1} c t^{-D^{\prime} / m}\left(2^{-1} u_{1}\right)^{-\left(\left|n_{1}\right|+\left|a_{2}^{\prime}\right|\right) / m}\left(2^{-1} u_{2}\right)^{-\left(\left|n_{2}^{\prime \prime}\right|+\left|a_{3}^{\prime}\right|\right) / m} \ldots \\
& \quad \cdots\left(2^{-1} u_{k-1}\right)^{-\left(\left|a_{k-1}^{\prime \prime}\right|+\left|a_{k}^{\prime}\right|\right) / m} u_{k}^{-\left|a_{k}^{\prime \prime}\right| / m} e^{\left.-b^{\prime}\left(\left.|| h\right|^{\prime}\right)^{m} t^{-1}\right)^{1 /(m-1)} e^{\omega^{\prime} t}}
\end{aligned}
$$

for all $h \in G$. Note that $\left|\alpha_{1}\right|+\left|\alpha_{2}^{\prime}\right| \leqq m-1, \cdots,\left|\alpha_{,-1}^{\prime \prime}\right|+\left|\alpha_{\jmath}^{\prime}\right| \leqq m-1,\left|\alpha_{.,}^{\prime \prime}\right|+\left|\alpha_{\jmath+1}^{\prime}\right|$ $\leqq m,\left|\alpha_{j+1}^{\prime \prime}\right|+\left|\alpha_{j+2}^{\prime}\right| \leqq m-1, \cdots,\left|\alpha_{k}^{\prime \prime}\right| \leqq m-1$, with obvious modifications if $j=1$ or $j=k$. So, with the hat denoting omission of the integral, one obtains 


$$
\begin{aligned}
& \int_{\langle 0, \iota, k-1} d u_{1} \cdots \widehat{d u_{j}} \cdots d u_{k}\left|\left(.4^{a_{1}} K_{u_{1}}^{g_{1}} \cdots \cdots *_{4} 4^{a} K_{u_{k}}^{g}\right)(h)\right| \\
& \leqq a^{2 k-1} c t^{-D^{\prime} / m} m^{k-1} k 2^{\left(\left|n_{1}\right|^{+}+\left|a_{k}\right|, / m\right.} t^{k-1-\left(\left|a_{1}\right|+\left|n_{2}\right|++\left|a_{k}\right|\right) / m} e^{-b^{\prime}\left(\left(|h|^{\prime}\right) n_{t-1}\right) 1 /(m-1)} e^{()^{\prime} \iota} \\
& \leqq 2^{k} a^{2 k-1} c t^{-D^{\prime} / m} e^{(k-1) t} m^{k-1} k t^{-1 a_{1} \mid / m} e^{-b^{\prime}\left(\left(|h|^{\prime}\right) m^{m} t-1,1 /(m-1)\right.} e^{\left(\nu^{\prime} t\right.} .
\end{aligned}
$$

This proves Statement I.

Next we prove Statement II. Since the estimates (11) are valid uniformly in $h$ and

$$
e^{-b^{\prime}\left(\left(|\eta|^{\prime}\right) m t-1,1,(m-1)\right.} \leqq 1
$$

it follows from the Lebesgue dominated convergence theorem that $F_{a_{1}}$ is continuous, hence $F_{a_{1}} \in C_{0}$. Similarly $F_{\beta} \in C_{0}$ for all $\beta \in J_{\left|a_{1}\right|}\left(d^{\prime}\right)$.

Now let $\beta \in J_{\left|a_{1}\right|-1}\left(d^{\prime}\right)$ and $i \in\left\{1, \cdots, d^{\prime}\right\}$. Then for all $h \in G$ and $s \in \boldsymbol{R}$, $s=0$ one has

$$
\begin{aligned}
& s^{-1}\left(F_{\beta}\left(\exp \left(-s a_{\imath}\right) / \iota\right)-F_{\beta}(h)\right)
\end{aligned}
$$

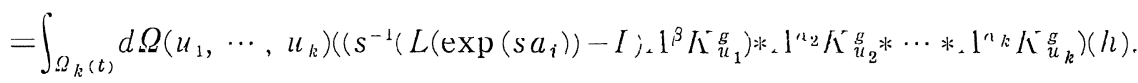

Now by the mean value theorem there exist $a, b, \omega>0$ such that for all $s \in$ $\langle 0,1] . h \in G$ and $\left.u_{1}\right\rangle 0$ there exists $s^{\prime} \in\langle 0, s\rangle$ such that

$$
\begin{aligned}
& \left|s^{-1}\left(\left(L\left(\exp \left(s a_{\imath}\right)\right)-I\right) \cdot 1^{3} K_{u_{1}}^{g}\right)(h)\right| \\
& \leqq a u_{1}^{-\left(N^{\prime}+1 s_{1+1}\right) / m} e^{\left(j u_{1}\right.} e^{\left.-b\left(\left(\left|\mathrm{e} \backslash \mathrm{p}\left(-s^{\prime} a_{l}\right) h^{\prime}\right|^{\prime}\right)\right)^{\prime} \iota-\mathrm{i}\right) 1\left(m^{\prime}-1\right.} \text {. }
\end{aligned}
$$

So arguing as above one obtains

$$
\lim _{s \rightarrow 0} s^{-1}\left(F_{\beta}\left(\exp \left(-s a_{1}\right) h\right)-F_{\beta}(h)\right)=F_{, l, \beta}(h)
$$

pointwise in $h$. But also

$$
\sup _{s \subseteq\langle 0,1]} \sup _{h \in G}\left|s^{-1}\left(F_{\beta}\left(\left(\exp _{(}-s a_{1}\right) / h\right)-F_{\beta}(h)\right)\right|<\infty \text {. }
$$

So $F_{\beta}$ is differentiable in the $L_{\infty}$-sense by [2] Proposition 3.1.23. Hence $F_{\beta}$ is differentiable in the $C_{0}$-sense. This proves Statement II.

Next let $g \equiv G$ and $t>0$. Then for all $\varphi \in L_{p}$ one has

$$
\begin{aligned}
\left(A^{3} K_{t}^{h}-A^{3} K_{t}^{g}\right) * \varphi & =A^{\beta} S_{t}^{h} \varphi-A^{\beta} S_{t}^{g} \varphi \\
& =-\int_{0}^{t} d u \cdot A^{\beta} S_{u}^{h}\left(H_{h}^{P}-H_{g}^{P}\right) S_{t-u}^{g} \varphi \\
& =-\sum_{a ;|a|=m}\left(C_{h}(\alpha)-C_{g}(\alpha)\right) \int_{0}^{t} d u A^{\beta} S_{u}^{h} A^{a} S_{t-u}^{g} \varphi \\
& =-\sum_{a ;|a|=m}\left(c_{a}(h)-c_{\alpha}(g)\right)\left(\int_{0}^{t} d u A^{\beta} K_{u}^{h} * A^{a} K_{t-u}^{g}\right) * \varphi,
\end{aligned}
$$

for all $h \in G$ where we have used Statement I and Fubini's theorem in the last 
equality. Therefore

$$
A^{\beta} K_{t}^{h}-A^{\beta} K_{t}^{g}=-\sum_{a,|a|=m}\left(c_{\alpha}(h)-c_{a}(g)\right) \int_{0}^{t} d u A^{\beta} K_{u}^{h} * A^{a} K_{t-u}^{g} .
$$

By Statement I there exists an $a>0$ such that

$$
\left\|\int_{0}^{t} d u A^{\beta} K_{u}^{h} * A^{a} K_{t-u}^{g}\right\|_{q} \leqq a
$$

for all $h \in G$ and $q \in[1, \infty]$. So

$$
\lim _{h \rightarrow g} A^{\beta} K_{t}^{h}=A^{\beta} K_{t}^{g}
$$

in $L_{1}$ and in $L_{\infty}$. But since $A^{\beta} K_{t}^{h} \in C_{0}$, the limit (14) is valid even in $C_{0}$.

Now we prove Statement III. For all $l \in\{1, \cdots, k\}$ let $\alpha_{l}^{\prime}, \alpha_{l}^{\prime \prime} \in J_{m-1}\left(d^{\prime}\right)$ be such that $\alpha_{l}=\left\langle\alpha_{l}^{\prime}, \alpha_{l}^{\prime \prime}\right\rangle$. Then for all $u_{1}, \cdots, u_{k+1}>0$

$$
\begin{aligned}
& \lim _{g_{1}, \cdots, g_{k} \rightarrow g} A^{\alpha_{1}} K_{u_{1}}^{g} g_{1 * \cdots *} A^{\alpha} K_{u_{k}}^{g}{ }_{u_{k}}
\end{aligned}
$$

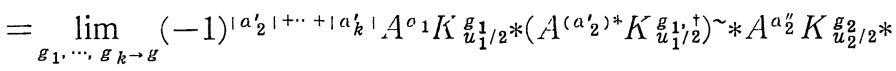

$$
\begin{aligned}
& \left(A^{\left(a_{3}^{\prime}\right) *} K_{u_{2}^{2} / 2}^{\left.g_{2}^{2}+\right)^{\prime}} \sim \cdots * A^{\alpha_{k}^{\prime \prime}} K_{u_{k}}^{g_{k}}\right. \\
& =\lim _{g_{1}, \cdots, g_{k \rightarrow g}}(-1)^{\left|\alpha_{2}^{\prime}\right|+\cdots+\left|a_{k}^{\prime}\right|} A^{a_{1}} K_{u_{1} / 2}^{g} *\left(A^{\left(a_{2}^{\prime}\right) * *} * K_{u_{1} / 2}^{g, \dagger}\right)^{\sim} * A_{2}^{a_{2}^{\prime \prime}} K_{u_{2} / 2}^{g} * \\
& \left(A^{\left(a_{3}^{\prime}\right) * T} K_{u_{2}^{\prime 2}}^{g, \dagger}\right)^{\sim} * \cdots * A^{\alpha_{k}^{\prime \prime}} K_{u_{k}}^{g}
\end{aligned}
$$

in $C_{0}$. So Statement III follows by the uniform estimate of (11) and the Lebesgue dominated convergence theorem.

Finally we prove the joint continuity of $(g, h) \mapsto K_{t}^{g}(h)$ for all $t>0$. Fix $t>0$. For all $g_{0}, h_{0}, g, h \in G$ one has

$$
\begin{aligned}
\left|K_{t}^{g}(h)-K_{t}^{g_{0}}\left(h_{0}\right)\right| & \leqq\left|K_{t}^{g}(h)-K_{t}^{g}(h)\right|+\left|K_{t}^{g}(h)-K_{t}^{g_{0}}\left(h_{0}\right)\right| \\
& \leqq\left\|K_{t}^{g}-K_{t}^{g_{0}}\right\|_{\infty}+\left|K_{t}^{g_{0}}(h)-K_{t}^{g_{0}}\left(h_{0}\right)\right| .
\end{aligned}
$$

Now $K_{t}^{g_{0}}$ is uniformly continuous and $\lim _{g \rightarrow g_{0}}\left\|K_{t}^{g}-K_{t}^{g_{0}}\right\|_{\infty}=0$ by $(14)$. So $(g, h)$ $\mapsto K_{l}^{g}(h)$ is continuous.

Proposition 2.3. Assume the conditions and notation of Lemma 2.2. Moreoner, suppose $c_{\alpha} \in C_{b ; m}^{\prime}$ for all $\alpha$ with $|\alpha|=m$.

I. For all $t>0, k \in\{0, \cdots, m-1\}$ and $l \in\{0, \cdots, m\}$ such that $k+l \leqq m$ the function $(g, h) \mapsto K_{t}^{g}(h)$ is $k$-times pointwise left-differentiable in $g$ and l-times left-differentiable in $h$, in any order, the derivatives are (jointly) continuous and the derivatives with respect to $g$ commute with those with respect to $h$.

II. Let $\beta \in J_{m-1}\left(d^{\prime}\right)$ and $\gamma \in J_{m}\left(d^{\prime}\right)$ and suppose that $|\beta|+|\gamma| \leqq m$. Then there exist $a, b, \omega>0$ such that

$$
\left|\left(A^{\beta} \partial^{r} K_{t}^{g}\right)(h)\right| \leqq a t^{-\left(D^{\prime}+|\beta|\right) / m} e^{\omega t} e^{-b\left(\left(|h|^{\prime}\right) m_{t-1}\right)^{1 /(m-1)}}
$$


for all $t>0$ and $g, h \in G$. The values of $a, b$ and $\omega$ depend on the coefficienls $c_{\alpha}$ through the ellipticity constant and the parameter $\sum_{\alpha ;|\alpha|=m}\left\|c_{a}\right\|_{\infty ;|r|}^{\prime}$.

Proof. Fix $\beta \in J_{m-1}\left(d^{\prime}\right)$. First we prove that for all $h \in G$ the function $g \mapsto\left(A^{\beta} K_{t}^{g}\right)(h)$ is $(m-|\beta|)$-times differentiable and that for all $\gamma \in J_{m-|\beta|}\left(d^{\prime}\right)$, $|\gamma| \neq 0$, all $k \in\{2, \cdots,|\gamma|+1\}$ and $\alpha_{2}, \cdots, \alpha_{k} \in J_{m}\left(d^{\prime}\right)$ there exist $c_{\alpha_{2}, \cdots, \alpha_{k}}^{\gamma, k} \in C_{b}$, polynomials of degree $k-1$ in the $A^{\delta} c_{\alpha}$ with $\delta \in J_{|r|}\left(d^{\prime}\right)$, such that

$$
\begin{aligned}
& \left(\partial^{\prime} A^{\beta} K_{t}^{g}\right)(h)=\sum_{k=2}^{\mid \gamma /+1} \sum_{a_{2} \cdot \cdots, a_{k}} c_{\alpha_{2}, \cdots, a_{k}}^{\gamma, k}(g) \\
& \cdot \int_{\Omega_{k}(t)} d \Omega\left(u_{1}, \cdots, u_{k}\right)\left(A^{\beta} K_{u_{1}}^{g} * A^{a_{2}} K_{u_{2}}^{g} * \cdots * A^{a_{k}} K_{u_{k}}^{g}\right)(h)
\end{aligned}
$$

for all $t>0$ and $g, h \in G$. The proof is by induction on $|\gamma|$.

Let $i \in\left\{1, \cdots, d^{\prime}\right\}, t>0$ and $g, h \in G$ and for $s \in \boldsymbol{R}$ set $g_{s}=\exp \left(-s a_{\imath}\right) g$. Then by (13) one has for $s \neq 0$

$$
\begin{aligned}
& s^{-1}\left(A^{\hat{\beta}} K_{t}^{g} s-. A^{\beta} K_{t}^{g}\right)(h) \\
& \quad=-\sum_{a ;|\alpha|=m} s^{-1}\left(c_{a}\left(g_{s}\right)-c_{\alpha}(g)\right) \int_{\Omega_{2}(t)} d \Omega\left(u_{1}, u_{2}\right)\left(A^{\beta} K_{u_{1}}^{g} \psi^{s} A^{n} K_{u_{2}}^{g}\right)(h) .
\end{aligned}
$$

Since the integral is a continuous function of $s$, by Lemma 2.2.III, the case $|\gamma|=1$ follows.

Now let $\gamma \in J_{m-|\beta|-1}\left(d^{\prime}\right),|\gamma| \geqq 1$ and suppose (15) is valid. Let $i \in\left\{1, \cdots, d^{\prime}\right\}$. Then for all $t>0$ and $g, h \in G$, with $g_{s}=\exp \left(-s a_{\imath}\right) g$ for all $s \in \boldsymbol{R}$, one has for $s \doteq 0$

$$
\begin{aligned}
& s^{-1}\left(\left(\partial^{r} A^{\beta} K_{t}^{g} s\right)(h)-\left(\partial^{\gamma} A^{\beta} K_{t}^{g}\right)(h)\right) \\
& =\sum_{k=2}^{|\gamma|+1} \sum_{a_{2}, \cdots, \alpha_{k}} s^{-1}\left(c_{\alpha_{2}, \cdots, a_{k}}^{\gamma, k}\left(g_{s}\right)-c_{a_{2}, \cdots, a_{k}}^{\gamma, k}(g)\right) \\
& \cdot \int_{\Omega_{k}(t)} d \Omega\left(u_{1}, \cdots, u_{k}\right)\left(A^{\beta} K_{u_{1}}^{g} A^{g} A^{a_{2}} K_{u_{2}}^{g} s_{*} * A^{\alpha} K_{k} K_{u_{k}}^{g s}\right)(h) \\
& +\sum_{k=2}^{|\gamma|+1} \sum_{a_{2}, \cdots, k_{k}} c_{\alpha_{2}, \cdots, a_{k}}^{\gamma, k}(g) s^{-1} \int_{\Omega_{k}(t)} d \Omega\left(u_{1}, \cdots, u_{k}\right) \\
& \cdot\left(\left(A^{\beta} K_{u_{1}}^{g} * A^{a_{2}} K_{u_{2}}^{g} * \cdots * A^{a_{k}} K_{u_{k}}^{g}\right)(h)-\left(A^{3} K_{u_{1}}^{g} * A^{a_{2}} K_{u_{2}}^{g} * \cdots * A^{a_{k}} K_{u_{k}}^{g}\right)(h)\right) \text {. }
\end{aligned}
$$

Now the integral in the first term is a continuous function of $s$ by Lemma 2.2.III. So by taking the limit $s \rightarrow 0$ we obtain the derivative of $c_{\alpha_{2}, \cdots, \alpha_{k}}^{\gamma, k}$. The subterms in the second sum need more care. Let $\alpha_{2}, \cdots, \alpha_{k} \in J_{m}\left(d^{\prime}\right)$ with $\left|\alpha_{2}\right|$ $=\cdots=\left|\alpha_{k}\right|=m$. For $l \in\{2, \cdots, k\}$ let $\alpha_{l}^{\prime}, \alpha_{l}^{\prime \prime} \in J_{m-1}\left(d^{\prime}\right)$ be such that $\alpha_{l}=\left\langle\alpha_{l}^{\prime}, \alpha_{l}^{\prime \prime}\right\rangle$ and

$$
\begin{aligned}
& \left|\alpha_{l}^{\prime}\right|=m-|\beta|-l+1, \\
& \left|\alpha_{l}^{\prime \prime}\right|=|\beta|+l-1 .
\end{aligned}
$$

This is possible since $2 \leqq l \leqq k \leqq m-|\beta|$. Then in $C_{0}(G)$ 


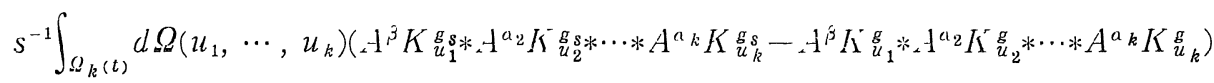

$$
\begin{aligned}
& =s^{-1}(-1)^{\left|a_{2}^{\prime} 1++\right| n_{k}^{\prime} \mid} \int_{\Omega_{k}(t)} d \Omega\left(u_{1}, \cdots, u_{k}\right) .
\end{aligned}
$$

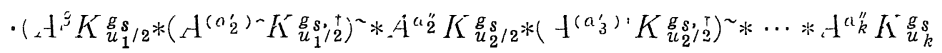

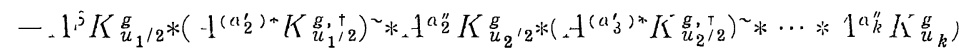

$$
\begin{aligned}
& =(-1)^{\left|a_{2}^{\prime}\right|++\left|a_{k}^{\prime}\right|} \int_{\Omega_{k}(t)} d Q\left(u_{1}, \cdots, u_{k}\right)
\end{aligned}
$$

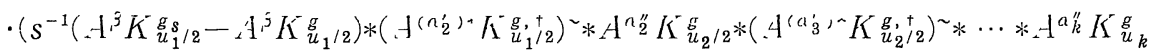

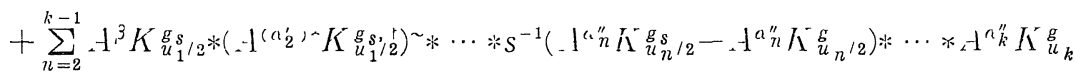

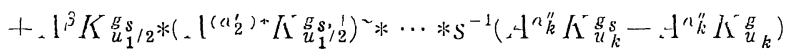

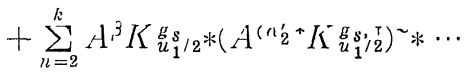

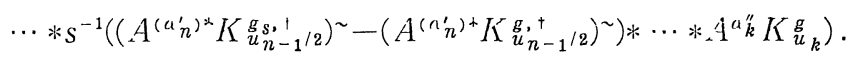

To estimate the term in the last sum one deduces by the argument used in the proof of equality (13), but with $g$ and $h$ interchanged, that

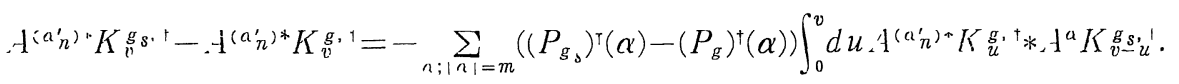

So

$$
\begin{aligned}
& \left(A^{\left(n_{n}^{\prime}\right)+} K_{v}^{g} \cdot+\right)^{\sim}-\left(A^{\left(a_{n}^{\prime}\right) \times} K_{v}^{g}{ }^{\dagger}\right)^{\sim}
\end{aligned}
$$

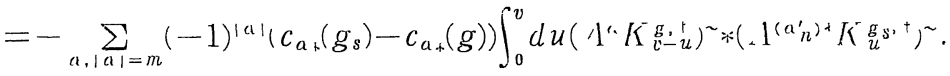

Therefore

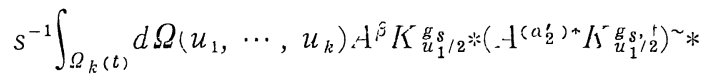

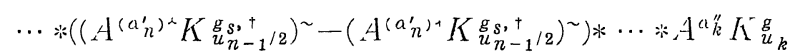

$$
\begin{aligned}
& =-\int_{\Omega_{k}(t)} d \Omega\left(u_{1}, \cdots, u_{k}\right) A^{\beta} K_{u_{1} / 2}^{g} *\left(A^{\left(a_{2}^{\prime}\right) *} K_{\left.\left.\frac{u_{1} / 2}{g}\right)^{\prime}\right)}{ }^{\sim} * \cdots\right. \\
& \cdots * s^{-1} \sum_{a ;|a|=m}(-1)^{|a|}\left(c_{a_{*} \mid}\left(g_{s}\right)-c_{\alpha *}(g)\right)
\end{aligned}
$$

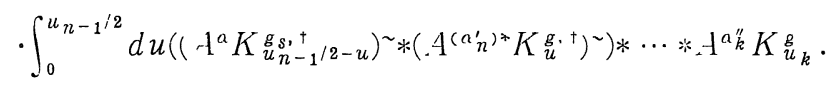

Now Lemma 2.2.I and a repeated use of (9) and (10) allows one to use Fubini's theorem and obtain 


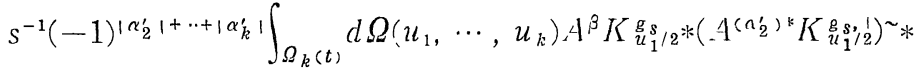

$$
\begin{aligned}
& \cdots *\left(\left(A^{\left(\kappa_{n}^{\prime}\right) *} K_{u_{n-1} / 2}^{g s, t}\right)^{\sim}-\left(\mathcal{A}^{\left(n_{n}^{\prime}\right)^{*}} K_{u_{n-1} / 2}^{g}\right)^{\sim, 1}\right) * \cdots * A^{a_{k}^{\prime \prime}} K_{u_{k}}^{g} \\
& =-\int_{\Omega_{k}(t)} d \Omega\left(u_{1}, \cdots, u_{k}\right) \int_{0}^{u_{n-1} / 2} d u \sum_{\alpha ;|\alpha|=m}\left(-1|| a|+| \alpha_{2}^{\prime}|+\cdots+| a_{k}^{\prime} \mid s^{-1}\left(c_{\alpha *}\left(g_{s}\right)-c_{a *}(g)\right)\right.
\end{aligned}
$$

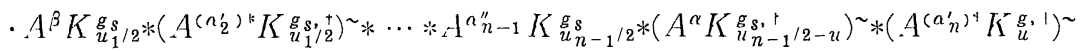

$$
\begin{aligned}
& * .1^{a \prime n} K_{u_{n} / 2}^{g} * \cdots * .4^{a \prime \prime} K_{u_{k}}^{g} \\
& =-\int_{\Omega_{k}(t)} d \Omega\left(u_{1}, \cdots, u_{k}\right) \int_{0}^{u_{n-1} / 2} d u \sum_{a:|a|=m} s^{-1}\left(c_{a+}\left(g_{s}\right)-c_{a *}(g)\right)
\end{aligned}
$$

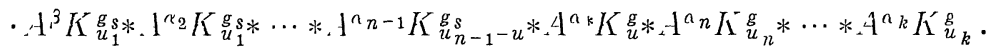

The other three terms in (16) can be handled in a similar, but slightly easier, manner and one obtains

$$
\begin{aligned}
& s^{-1} \int_{\Omega_{k}(t)} d \Omega\left(u_{1}, \cdots, u_{k}\right)\left(A^{\beta} K_{u_{1}}^{g} s_{1} * \Lambda^{\alpha_{2}} K_{u_{2}}^{g} s_{2} * * \Lambda^{n} k K_{u_{k}}^{g} s-\Lambda^{\beta} K_{u_{1}}^{g} * A^{n_{2}} K_{u_{2}}^{g} * \cdots * \Lambda^{n} k K_{u_{k}}^{g}\right) \\
& =-\int_{\Omega_{k}(t)} d \Omega\left(u_{1}, \cdots, u_{k}\right) \int_{0}^{u_{n}} d u \sum_{n=2}^{k} \sum_{n ;|a|=m} s^{-1}\left(c_{n+}\left(g_{s}\right)-c_{\alpha \cdot}(g)\right)
\end{aligned}
$$

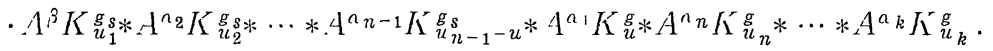

A new application of Lemma 2.2 establishes that the limit $s \rightarrow 0$ exists and the induction step follows.

In case $\beta \in J_{m-2}\left(d^{\prime}\right), \gamma \in J_{m}\left(d^{\prime}\right), i \in\left\{1, \cdots, d^{\prime}\right\}$ and $|\beta|+|\gamma| \leqq m-1$ it follows from (15) and Lemma 2.2.II that $h \mapsto\left(\partial^{i} A^{3} K_{t}^{g}\right)(h)$ is differentiable and $\left(A_{2} \partial^{r} A^{\beta} K_{t}^{g}\right)(h)=\left(\partial^{r} \cdot A_{2} \cdot A^{\beta} K_{l}^{g}\right)(h)$. So the derivatives with respect to $g$ and $h$ commute.

Finally, the continuity of $(g, h) \mapsto K_{t}^{g}(h)$ follows as in the proof of Lemma 2.2.IV, using (15) and Lemmas 2.2.II and 2.2.III. 'This proves the proposition.

Now we are prepared to give the proof of Theorem 2.1. Recall, we have a subcoercive form $C$, possibly with lower order coefficients, $P$ is the principal part of $C, H=d L(C), H^{\dagger}=d L\left(C^{\dagger}\right), H_{g}=d L\left(C_{g}\right)$ and $K_{t}^{g}$ is the kernel of the semigroup $S_{t}^{g}$ generated by the closure of the operator $H_{g}^{P}=d L\left(P_{g}\right)$, the principal part of $H_{g}$. The proof is divided in several steps.

Step 1. It follows from Proposition 2.3 that there exist $a, b, \omega>0$ such that

$$
\left|\left(A^{\beta} \partial^{i} K_{t}^{g}\right)\left(g h^{-1}\right)\right| \leqq a t^{-\left(D^{\prime}+1,31\right) / m} e^{\omega t} e^{-b\left(\left(\left.1 g h^{-1}\right|^{\prime}\right) m t-1,1 /(m-1)\right.}
$$

for all $\beta \in J_{m-1}\left(d^{\prime}\right), \gamma \in J_{m}\left(d^{\prime}\right)$ and $t>0$ such that $|\beta|+|\gamma| \leqq m$. So one can define the function $L_{t}, t \in \boldsymbol{R}$ by (3). Note that for $t \leqq 0$ the definition of $L_{t}$ obviously 
makes sense since $K_{t}^{g}=0$ for $t \leqq 0$, in particular for $t=0$. For all $t \in \boldsymbol{R}$ and $h \in G$ fixed the function $\phi$ on $G$ defined by $\psi(g)=K_{t}^{g}\left(g h^{-1}\right)$ is $m$-times pointwise differentiable and

$$
(H \psi)(g)=\left(H_{g}^{P} K_{t}^{g}\right)\left(g h^{-1}\right)+L_{t}(g ; h)
$$

for all $g \in G$. Here the operator $H$ in $H \psi$ is the pointwise defined differential operator and the operator $H_{g}^{P}$ in $\left(H_{g}^{P} K_{t}^{g}\right)\left(g h^{-1}\right)$ is the principal part of the operator $H_{g}$ acting on the function $K_{t}^{g} \in C_{0}(G)$. So for all $\varphi \in C_{c}^{\infty}(\boldsymbol{R} \times G \times G)$ one obtains by the substitution $\phi(t, g, h)=\varphi\left(t, g, h^{-1} g\right)$ and partial integration

$$
\begin{aligned}
& \int_{R} d t \int_{G} d \hat{h} \int_{G} d g\left(\left(-\partial_{t}+H^{\dagger}\right) \varphi\right)(t, g, h) K_{t}^{(0)}(g ; h) \\
& =-\int_{R} d t \int_{G} d \hat{h} \int_{G} d g\left(\partial_{t} \varphi\right)(t, g, h) K_{t}^{g}\left(g h^{-1}\right) \\
& \quad+\int_{R} d t \int_{G} d \hat{h} \int_{G} d g \varphi(t, g, h)\left(\left(H_{g}^{P} K_{t}^{g}\right)\left(g h^{-1}\right)+L_{t}(g ; h)\right) \\
& =-\int_{R} d t \int_{G} d h \int_{G} d g\left(\partial_{t} \psi\right)(t, g, h) K_{t}^{g}(h)+\int_{R} d t \int_{G} d h \int_{G} d g \phi(t, g, h)\left(H_{g}^{P} K_{t}^{g}\right)(h) \\
& \quad+\int_{R} d t \int_{G} d \hat{h} \int_{G} d g \varphi(t, g, h) L_{t}(g ; h) \\
& =\int_{G} d g \int_{R} d t \int_{G} d h\left(-\partial_{t}+\left(H_{g}^{P,}(h)\right)^{*}\right) \psi(t, g, h) K_{t}^{g}(h) \\
& \quad+\int_{R} d t \int_{G} d \hat{h} \int_{G} d g \varphi(t, g, h) L_{t}(g ; h) \\
& =\int_{G} d g \phi(0, g, e)+\int_{R} d t \int_{G} d \hat{h} \int_{G} d g \varphi(t, g, h) L_{t}(g ; h) \\
& =\int_{G} d g \varphi(0, g, g)+\int_{R} d t \int_{G} d \hat{h} \int_{G} d g \varphi(t, g, h) L_{t}(g ; h) \\
& =\int_{R} d t \int_{G} d \hat{h} \int_{G} d g \varphi(t, g, h)\left(\delta(t) \delta\left(g h^{-1}\right)+L_{t}(g ; h)\right)
\end{aligned}
$$

where $H_{g}^{P,(h)}$ denotes the operator $H_{g}^{P}$ acting on the $h$-variable. So the differential equation (2) is valid as distribution on $\boldsymbol{R} \times G \times G$, with measure $d t \times$ $d g \times d \hat{h}$. Furthermore, one obtains the pointwise estimates

$$
\begin{aligned}
& \left|K_{t}^{(0)}(g ; h)\right| \leqq a t^{-D^{\prime} / m} e^{\omega t} e^{-b\left(\left(\left|g h^{-1}\right|^{\prime}\right)^{m} t^{-1}\right)^{1 /(m-1)}} \\
& \left|L_{t}(g ; h)\right| \leqq a t^{-D^{\prime} / m} t^{-(m-1) / m} e^{\omega t} e^{-b\left(\left(\left|g h^{-1}\right|^{\prime}\right) m^{2} t^{-1}\right) 1 /(m-1)}
\end{aligned}
$$

valid for all $t>0$ and $g, h \in G$, with redefined $a, b, \omega$. Hence, by increasing $a$ and $\omega$ if necessary, one establishes bounds

$$
\left\|K_{t}^{(0)}\right\|_{1}^{\rho} \leqq a e^{\omega(1+\rho m) t} \quad\left\|L_{t}\right\|_{1}^{\rho} \leqq a t^{-(m-1) / m} e^{\omega\left(1+\rho^{m}\right) t}
$$




$$
\begin{gathered}
\left\|K_{t}^{(0)}\right\|_{\infty}^{\rho} \leqq a t^{-D^{\prime} / m} e^{\omega(1+\rho m) t} \quad\left\|L_{t}\right\|_{\infty}^{\rho} \leqq a t^{-D^{\prime} / m} t^{-(m-1) / m} e^{\omega(1+\rho) m) t} \\
\left\|L_{t}\right\|_{\tilde{1}}^{\rho} \leqq a t^{-(m-1) / m} e^{\omega(1+\rho m) t}
\end{gathered}
$$

for all $\rho \geqq 0$ and $t>0$ and with $a$ and $\omega$ independent of $\rho$ and $t$.

Now arguing as in the proof of the proposition in the appendix of [4] and Theorem 7.1 in [10] we conclude that indeed the definition (5) of $K_{t}^{(n)}$ makes sense, the function $K_{t}^{(n)}$ is continuous for all $t>0$ and $n \in \boldsymbol{N}_{0}$, the series (4) for $K_{t}$ converges in $\mathcal{L}_{1}^{o}$ and $\mathcal{L}_{\infty}^{o}$, the function $K_{t}$ is continuous for all $t>0$ and $K_{t}$ satisfies the bounds of Statement VI of Theorem 2.1 if $|\alpha|=n=0$. In the derivation of these properties we have only used the $m$-th order derivatives of the $c_{\alpha}$ with $|\alpha|=m$ and the bounds only depend on the ellipticity constant and the parameter $\|C\|_{m}^{\prime}$. It then follows by construction that $K$ satifies the heat equation (1) and $K_{t}=0$ for $t \leqq 0$. One can deduce even more. The function $t \mapsto K_{t}$ on $\{t \in \boldsymbol{R}: t>0\}$ has an extension to a sector $\Delta_{\theta}$ with $\theta \geqq \theta_{C}$. This follows because each of the operators $H_{\theta}=e^{2 \theta} H,|\theta|<\theta_{C}$, is subcoercive and hence the foregoing construction applied to $H_{\theta}$ yields a solution $K^{\theta}$ of the corresponding heat equation. But then the extension of $K$ is defined by $K_{e^{2} \theta_{t}}=K_{t}^{\theta}$ for $t>0$.

This construction of the kernel for complex $t$ also implies that the "Gaussian" bounds on $K$ extend to the interior of the sector $\Delta_{\theta_{C}}$. The bounds on $\left|K_{z}\right|$ with $z=e^{i \theta} t, t>0$, follow by consideration of the operator $H_{\theta}$ if $|\theta|<\theta_{C}$. But the bounds can be chosen uniform in $\theta$ if $|\theta| \leqq \varphi<\theta_{C}$. Specifically, there are $a, b>0$ and $\omega \geqq 0$ such that

$$
\left|K_{z}(g ; h)\right| \leqq a|z|^{-D^{\prime} / m} e^{\omega|z|} e^{-b\left(\left(|g|^{\prime}\right) m_{\left.|z|^{-1}\right) 1 /(m-1)}\right.}
$$

for all $g, h \in G$ and all non-zero $z \in \Delta_{\varphi}$ with $\varphi<\theta_{C}$ where $a, b$ and $\omega$ again depend on the coefficients only through the ellipticity constant and the parameter $\|C\|_{m}^{\prime}$.

At this point we have constructed $K$ as a solution of the appropriate heat equation and if $t>0$ the corresponding function is an obvious candidate for the semigroup kernel. Nevertheless we have not established that $K$ is a semigroup under convolution and it does not seem that the parametrix expansion is particularly suited to the proof of algebraic properties. Therefore we adopt a different tactic and use $K^{g}$ to establish that the closures of $H$ generate interpolating semigroups on the $L_{p^{-}}$, and $L_{\hat{p}^{-}}$, spaces. Then we exploit this information to prove that the $K_{t}$ with $t>0$ is a convolution semigroup.

Step 2. Since $K^{g}$ satisfies 'Gaussian' bounds one may define the kernel

$$
R_{\lambda}^{g}=\int_{0}^{\infty} d t e^{-\lambda t} K_{t}^{g}
$$

for all $\lambda \in C$ with $\operatorname{Re} \lambda>\omega$ and introduce the corresponding operator $r_{\lambda}^{(0)}$ on the $L_{p^{-}}$, or $L_{\hat{p}^{-}}$, spaces by 


$$
\left(r_{\lambda}^{(0)} \varphi\right)(g)=\left(R_{\lambda}^{g} * \varphi\right)(g) .
$$

But the 'Gaussian' bounds on $K_{t}^{g}$ are uniform in $g$ and hence the operator $r_{i}^{(0)}$ satisfies the bounds

$$
\left\|r_{\lambda}^{(0)}\right\|_{p \rightarrow p} \leqq c(\operatorname{Re} \lambda)^{-1}, \quad\left\|r_{\lambda}^{(0)}\right\|_{\hat{p} \rightarrow \hat{p}} \leqq c(\operatorname{Re} \lambda)^{-1}
$$

for a suitable $c>0$ and all $\lambda \in C$ with $\operatorname{Re} \lambda>\omega$ and $\omega$ sufficiently large. The values of $c$ and $\omega$ depend continuously on the ellipticity constant $\mu$ and $\left\|c_{\alpha}\right\|_{\infty}$ with $|\alpha|=m$.

Next we prove that $r_{\lambda}^{(0)}$ maps $C_{c}^{\infty}(G)$ into $L_{p ; m}^{\prime} \cap L_{\hat{p} ; m}^{\prime}$ if $\operatorname{Re} \lambda$ is large enough. Let $\alpha \in J_{m}\left(d^{\prime}\right)$ and $\varphi \in C_{c}^{\infty}(G)$. Then with $A^{a}$ denoting the pointwise derivatives one obtains for all $g \in G$ by (15) and the bounds of Lemma 2.2

$$
\begin{aligned}
\left(\Lambda^{n} r_{\dot{\lambda}}^{(0)} \varphi\right)(g) & =\sum_{(\beta, \gamma) \in L b(a)} \int_{0}^{\infty} d t \int_{G} d h e^{-\lambda t}\left(\hat{o}^{\beta} K_{t}^{g}\right)(h)\left(A^{i} L(h) \varphi\right)(g) \\
& =\sum_{(\beta, ;) \in L^{b}(a)} \int_{0}^{\infty} d t \int_{G} d h e^{-\lambda t}\left(\partial^{\beta} K_{t}^{g}\right)(h)\left(\left(L\left(h^{-1}\right) A^{i} L(h)\right) \varphi\right)\left(h^{-1} g\right)
\end{aligned}
$$

where the sum is over all pairs of multi-indices $L b(\alpha)$ arising from the Leibniz formula for the multi-derivative $A^{a}$ of the product. There exist $M, \sigma \geqq 0$, and for each $r \in J_{m}\left(d^{\prime}\right)$ and $\varepsilon \in J_{1, r r}\left(d^{\prime}\right)$ there exists $c_{1, \varepsilon} \in C^{\infty}(G)$, such that for all $h \in G$

$$
L\left(h^{-1}\right) \cdot A^{\prime} L(h)=\sum_{\left.\varepsilon \in J|\gamma| r^{(} d^{\prime}\right)} c_{\gamma, \varepsilon}(h) \Lambda^{\varepsilon}
$$

with $\left|c_{7, \varepsilon}(h)\right| \leqq M e^{\sigma|h|^{\prime}}$ where $r$ is the rank of the algebraic basis $a_{1}, \cdots, a_{d^{\prime}}$. Then

$$
\left(A^{n} r_{\lambda}^{(0)} \varphi\right)(g)=\sum_{(\beta, \eta) \in L b(a)} \sum_{\varepsilon \in J} \sum_{|\gamma| r^{\left(d^{\prime}\right)}} \int_{0}^{\infty} d t \int_{G} d h e^{-\lambda t}\left(\partial^{3}{ }^{3} K_{l}^{g}\right)(h) c_{\eta, \varepsilon}(h)\left(A^{\varepsilon} \varphi\right)\left(h^{-1} g\right) .
$$

So

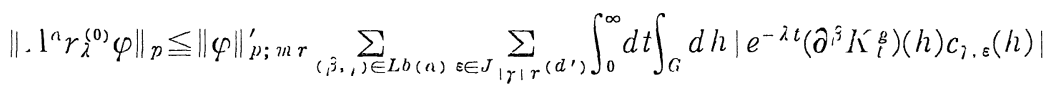

$$
\begin{aligned}
& \leqq a M\|\varphi\|_{p ; m r}^{\prime} \sum_{(, \beta, \eta) \in L b(a)} \sum_{s \in J} \sum_{|\gamma| r^{\left(d^{\prime}\right)}} \int_{0}^{\infty} d t \int_{G} d h e^{-\lambda t} t^{-D^{\prime} / m} e^{\omega t} e^{-b\left((\mid h)^{\prime}\right)^{m} t-1,1,(m-1)} e^{\sigma\left|h^{\prime}\right|} \\
& \leqq c(\operatorname{Re} \lambda)^{-1}\|\varphi\|_{p ; m r}^{\prime}<\infty
\end{aligned}
$$

for some $c>0$ and $\operatorname{Re} \lambda$ sufficiently large. Moreover, $A^{a} r_{\lambda}^{(0)} \varphi$ is continuous for all $\alpha \in J_{m}\left(d^{\prime}\right)$. So $r_{\lambda}^{(0)} \varphi \in L_{p ; m}^{\prime}$. Since $\Delta(g)$ grows at most exponentially in $|g|^{\prime}$, it follows similarly that $r_{\lambda}^{(0)} \varphi \in L_{\hat{p}}^{\prime} ; m$.

Now let $\varphi \in C_{c}^{\infty}(G), \lambda \in C$ and suppose $\operatorname{Re} \lambda$ is sufficiently large. For $N \in N$ let $\chi_{s} \in C_{c}^{\infty}(\boldsymbol{R})$ satisfy $0 \leqq \chi_{N} \leqq 1, \chi_{N}(t)=1$ if $t \in[0, N]$, supp $\chi_{N} \leqq\left[-N^{-1}, N+1\right]$ and $\chi_{N}(N+t)=\chi_{1}(1+t)$ for all $t \in[0,1]$. Moreover, let $e_{\lambda}(t)=e^{-\lambda . t}$. Then for all $\phi \in C_{c}^{\infty}(G)$ 
$\left(\psi,(\lambda I+H) r_{\lambda}^{(0)} \varphi\right)=\left(\left(\bar{\lambda} I+H^{\dagger}\right) \psi, r_{\lambda}^{(0)} \varphi\right)$

$$
\begin{aligned}
= & \int_{G} d g \int_{G} d \hat{h} \int_{0}^{\infty} d \overline{t\left(\left(\bar{\lambda} I+H^{\dagger}\right) \psi\right)(g)} e_{\lambda}(t) K_{t}^{g}\left(g h^{-1}\right) \varphi(h) \\
= & \lim _{N \rightarrow \infty} \int_{G} d g \int_{G} d \hat{h} \int_{0}^{\infty} d \overline{\left.t\left(\bar{\lambda} I+H^{\dagger}\right) \psi\right)(g)} e_{\lambda}(t) \chi_{N}(t) \varphi(h) K_{t}^{g}\left(g h^{-1}\right) \\
= & \lim _{N \rightarrow \infty} \int_{G} d g \int_{G} d \hat{h} \int_{0}^{\infty} d \overline{t\left(\left(-\hat{o}_{t}+H^{\dagger}\right)\left(\phi \otimes \bar{\varphi} \otimes\left(e_{\bar{\lambda}} \chi_{N}\right)\right)\right)(g, h, t)} K_{t}^{g}\left(g h^{-1}\right) \\
& +\lim _{N \rightarrow \infty} \int_{G} d g \int_{G} d \hat{h} \int_{0}^{\infty} d t \overline{\left(\phi \otimes \bar{\varphi} \otimes\left(e_{\bar{\lambda}} \chi_{N}^{\prime}\right)\right)(g, h, t)} K_{t}^{r}\left(g h^{-1}\right) \\
= & \lim _{N \rightarrow \infty} \int_{G} d g \int_{G} d \hat{h} \int_{0}^{\infty} d t\left(\bar{\psi} \otimes \varphi \otimes\left(e_{\bar{\lambda}} \chi_{N}\right)\right)(g, h, t)\left(\delta(t) \delta\left(g h^{-1}\right)+L_{t}(g ; h)\right) \\
= & (\psi, \varphi)+\left(\psi, Q_{\lambda} \varphi\right),
\end{aligned}
$$

where for all $\varphi \in L_{p}$ we have defined $Q_{\lambda} \varphi \in L_{p}$ by

$$
\left(Q_{\lambda} \varphi\right)(g)=\int_{G} d \hat{h} \int_{0}^{\infty} d t e^{-\lambda t} L_{t}(g ; h) \varphi(h) .
$$

But since $L_{\iota}$ satisfies 'Gaussian' bounds, the operator $Q_{\lambda}$ is continuous on $L_{p}$ and

$$
\left\|Q_{\lambda}\right\|_{p \rightarrow p} \leqq c(\operatorname{Re} \lambda)^{-1 / m}
$$

for some $c>0$ and all $\lambda \in C$ with $\operatorname{Re} \lambda$ sufficiently large. So

$$
\left(\phi,(\lambda I+\bar{H}) r_{\lambda}^{(0)} \varphi\right)=(\phi, \varphi)+\left(\phi, Q_{\lambda} \varphi\right)
$$

for all $\varphi, \phi \in C_{c}^{\infty}(G)$. Since $C_{c}^{\infty}(G)$ is $\sigma\left(L_{q}, L_{p}\right)$-dense in $L_{q}$, the equation (20) is valid for all $\phi \in L_{q}$ and $\varphi \in C_{c}^{\infty}(G)$. But since the right hand side of (20) depends $\sigma\left(L_{p}, L_{q}\right)$-continuously on $\varphi$, it follows that $r_{\lambda}^{(0)} \varphi \in D(\bar{H})$ for all $\varphi \in L_{p}$ and

$$
(\lambda I+\bar{H}) r_{\lambda}^{(0)} \varphi=\varphi+Q_{\lambda} \varphi
$$

for all $\varphi \in L_{p}$. In particular, if $\operatorname{Re} \lambda$ is so large that $\left\|Q_{\lambda}\right\|_{p \rightarrow p}<1$, the operator $I+Q_{\lambda}$ is invertible, and the range $R(\lambda I+\bar{H})$ of the operator $\lambda I+\bar{H}$ is $L_{p}$.

Similarly one may construct operators $r_{\lambda}^{(0)+1}$ and $Q_{\lambda}^{\dagger}$ starting from $H^{\dagger}$ and one finds

$$
\left(\bar{\lambda} I+\overline{H^{\dagger}}\right) r_{\bar{\lambda}}^{(0), \dagger}=I+Q_{\lambda}^{\dagger}
$$

on $L_{q}$. So

$$
\left(r_{\bar{\lambda}}^{(0), \dagger}\right)^{*}\left(\lambda I+H^{\dagger *}\right) \varphi=\left(I+\left(Q_{\lambda}^{\dagger}\right)^{*}\right) \varphi
$$

for all $\varphi \in D\left(\lambda I+H^{+^{*}}\right)$. But $H^{\dagger^{*}}$ is an extension of $\bar{H}$, so

$$
\begin{aligned}
\left\|\left(r_{\bar{\lambda}}^{(0), 1}\right)^{*}\right\|_{p \rightarrow p}\|(\lambda I+\bar{H}) \varphi\|_{p} & \geqq\left(1-\left\|\left(Q_{\lambda}^{4}\right)^{*}\right\|\right)\|\varphi\|_{p} \\
& \geqq 2^{-1}\|\varphi\|_{p}
\end{aligned}
$$

if $\operatorname{Re} \lambda$ is sufficiently large. Consequently one has bounds 


$$
\|(\lambda I+\bar{H}) \varphi\|_{p} \geqq c(\operatorname{Re} \lambda)\|\varphi\|_{p}
$$

for all $\lambda \in C$ with $\operatorname{Re} \lambda \geqq \omega_{0}$ with $\omega_{0}$ sufficiently large, since $\left\|\left(r_{\frac{1}{\lambda}}^{(0)}, \mathrm{r}\right)^{*}\right\|_{p \rightarrow p}=$ $\left\|r_{\bar{\lambda}}^{(0),+}\right\|_{q \rightarrow q} \leqq c^{\prime}(\operatorname{Re} \lambda)^{-1}$ if $\operatorname{Re} \lambda \geqq \omega_{0}$. But if $H$ is replaced by $e^{i \theta} H$ with $|\theta|<\theta_{C}$ one can reason similarly and finally deduce as in the proof of [10] Theorem 5.2 that $\bar{H}$ generates a holomorphic semigroup on $L_{p}$ with holomorphy angle at least $\theta_{C}$. Similarly, the $L_{\hat{p}}$-closure of the operator $H$ in $L_{p}$ generates a holomorphic semigroup with holomorphy angle at least as large as $\theta_{C}$.

It follows as above that $\overline{H^{\dagger}}$ also generates a holomorphic semigroup $S^{\dagger}$. Then $H^{\dagger^{*}}$ generates a holomorphic semigroup $S^{{ }^{*}}$. But $\bar{H} \cong H^{\dagger^{*}}$ and since a generator cannot have a strict extension it follows that $\bar{H}=H^{\dagger^{*}}$.

Step 3. Next we prove Statement III. The idea is to identify the kernel of the semigroup with the distribution $K$ constructed by the parametrix expansion. First we use $K$ to define a family of bounded operators. For $t \geqq 0$ and $\varphi \in L_{p}$ define $T_{t} \varphi$ by

$$
\left(T_{t} \varphi\right)(g)=\int_{G} d \hat{h} K_{t}(g ; h) \varphi(h) .
$$

Then by the 'Gaussian' bounds on $K_{t}$ we see that $T_{t}$ maps $L_{1}$ continuously into $L_{1}$ and $L_{\infty}$ continuously into $L_{\infty}$, so by interpolation $T_{t}$ maps $L_{p}$ continuously into $L_{p}$. Moreover, $\left\|T_{t}\right\|_{p \rightarrow p}$ satisfies bounds $\left\|T_{t}\right\|_{p \rightarrow p} \leqq a e^{\omega t}$ for some $a, \omega>0$. Since $K$ satisfies 'Gaussian' bounds one may define the kernel $R_{\lambda}$ by

$$
R_{\lambda}(g ; h)=\int_{0}^{\infty} d t e^{-\lambda t} K_{t}(g ; h)
$$

for all $\lambda \in C$ with $\operatorname{Re} \lambda$ large enough and introduce the corresponding operator $r_{\lambda}$ by

$$
\left(r_{\lambda} \varphi\right)(g)=\int_{G} d \hat{h} R_{\lambda}(g ; h) \varphi(h) .
$$

Then $r_{\lambda}$ is continuous and by a calculation similar to that used to deduce (19) one proves that

$$
\left(\left(\bar{\lambda} I+H^{\dagger}\right) \psi, r_{\lambda} \varphi\right)=(\phi, \varphi)
$$

for all $\varphi, \phi \in C_{c}^{\infty}(G)$. Since $C_{c}^{\infty}(G)$ is $\sigma\left(L_{p}, L_{q}\right)$-dense in $L_{p}$, where $q$ is dual to $p$, it follows that (21) is valid for all $\varphi \in L_{p}$ and $\phi \in C_{c}^{\infty}(G)$.

At this stage we need a lemma which can be stated in a much wider context.

Lemma 2.4. Let $(\mathfrak{X}, G, U)$ be a strongly, or weakly*, continuous representation and further let $a_{1}, \cdots, a_{a^{\prime}}$ be an algebraic basis in the Lie algebra $g$ of $G$. Let $m \in \boldsymbol{N}$. Then for all $x \in \mathfrak{X}_{m}^{\prime}$ there exists a sequence $x_{1}, x_{2}, \cdots \in \mathfrak{X}_{\infty}$ such that for all compact $K \subset \mathcal{F}$, the dual or predual, of $\mathscr{X}$, and all $\alpha \in J_{m}\left(d^{\prime}\right)$ 


$$
\lim _{n \rightarrow \infty}\left(f, A^{a} x_{n}\right)=\left(f, A^{\alpha} x\right),
$$

uniformly for all $f \in K$. In particular if the representation is strongly continuous then $\mathfrak{X}_{\infty}$ is norm dense in $\mathfrak{X}_{m}^{\prime}$.

Proof. Let $\tau_{1}, \tau_{2}, \cdots$ be the bounded approximation of the identity as defined before Lemma 3.21 in [10]. Also, for $\varphi \in C_{c}^{\infty}(G)$ and $\alpha=\left(i_{1}, \cdots, i_{k}\right) \in$ $J\left(d^{\prime}\right)$ let $M_{\alpha} \varphi \in C_{c}^{\infty}(G)$ be defined as before Lemma 3.20 in [10]. Then by $[10]$ Lemma 3.20

$$
\begin{aligned}
U\left(M_{\alpha} \varphi\right) x & =(\operatorname{ad} A)^{a}(U(\varphi)) x \\
& =\left[A_{i_{1}},\left[A_{\imath_{2}},\left[\cdots,\left[A_{i_{k}}, U(\varphi)\right] \cdots\right]\right] x\right.
\end{aligned}
$$

for all $x \in \mathfrak{X}_{k}^{\prime}$, where

$$
U(\psi) x=\int_{G} d g \psi(g) U(g) x
$$

for all $\phi \in C_{c}^{\infty}(G)$. The important fact is that there exists $c>0$ such that $\left\|M_{\alpha} \tau_{n}\right\|_{1} \leqq c$ for all $\alpha \in J_{m}\left(d^{\prime}\right)$ and $n \in N$ and $\bigcup_{n \in N} \operatorname{supp} \tau_{n}$ is compact (see [10] Lemma 3.21).

Let $\alpha \in J_{m}\left(d^{\prime}\right),|\alpha| \pm 0$ and write $\alpha=\left(i_{1}, \cdots, i_{k}\right)$. Then for all $x \in \mathfrak{X}_{|\alpha|+1}$ one has

$$
\begin{aligned}
\lim _{n \rightarrow \infty}\left\|U\left(M_{\alpha} \tau_{n}\right) x\right\| & =\lim _{n \rightarrow \infty}\left\|(\operatorname{ad} A)^{\alpha}\left(U\left(\tau_{n}\right)\right) x\right\| \\
& =\lim _{n \rightarrow \infty}\left\|(\operatorname{ad} A)^{\alpha}\left(V\left(\tau_{n}\right)\right) x\right\|=0,
\end{aligned}
$$

where $V$ is the strongly continuous representation obtained by restricting $U$ to the $C^{|\alpha|}$-elements of the strongly continuous component of $U$ and a full vector space basis of g. But since $\left\|U\left(M_{\alpha} \tau_{n}\right)\right\| \leqq c^{\prime}\left\|M_{\alpha} \tau_{n}\right\|_{1} \leqq c c^{\prime}$ for all $n \in N$, with $c^{\prime}=$ $\sup \left\{\|U(g)\|: g \in \cup_{n \in N} \operatorname{supp} \tau_{n}\right\}<\infty$ and $\mathscr{X}_{|\alpha|+1}$ is dense in $\mathscr{X}$ with repect to the topology of uniform convergence on compact subsets of $\mathscr{I}$ (see [8], proof of Assertion 2) it follows that for all $x \in \mathscr{X}$ and compact $K \subset \mathscr{F}$

$$
\lim _{n \rightarrow \infty}\left(f, U\left(M_{\alpha} \tau_{n}\right) x\right)=0,
$$

uniformly for $f \in K$.

Now let $\alpha \in J_{m}\left(d^{\prime}\right)$ and $x \in \mathfrak{X}_{m}^{\prime}$. Then

$$
A^{\alpha} U\left(\tau_{n}\right) x=\sum_{(\beta, \gamma) \in L b(\alpha)} U\left(M_{\gamma} \tau_{n}\right) A^{\beta} x
$$

where the sum is again over the multi-indices occurring in the Leibniz formula for the multi-derivative $A^{\alpha}$ of a product. So for all compact $K \subset \mathscr{F}$ one has

$$
\begin{aligned}
\lim _{n \rightarrow \infty}\left(f, A^{\alpha} U\left(\tau_{n}\right) x\right) & =\lim _{n \rightarrow \infty}\left(f, U\left(\tau_{n}\right) A^{\alpha} x\right)+\sum_{\substack{(\beta, \gamma) \in L b(\alpha) \\
\beta \neq \alpha}} \lim _{n \rightarrow \infty}\left(f, U\left(M_{\gamma} \tau_{n}\right) A^{\beta} x\right) \\
& =\left(f, A^{\alpha} x\right)
\end{aligned}
$$


uniformly for $f \in K$.

If the representation is strongly continuous all limits are uniform in $f$ and $\mathscr{X}_{\infty}$ is norm dense in $\mathscr{X}_{m}^{\prime}$.

We continue with the proof of Statement III of Theorem 2.1. By Lemma 2.4 it follows that (21) is valid for all $\varphi \in L_{p}$ and $\phi \in L_{q ; m}^{\prime}$. So $r_{\lambda} \varphi \in D\left(\left(\bar{\lambda} I+H^{\dagger}\right)^{*}\right)$ and $\left(\lambda I+H^{\dagger *}\right) r_{\lambda} \varphi=\varphi$. But $H^{+*}=\bar{H}$, so if $\operatorname{Re} \lambda$ is large enough one has $r_{\lambda}=$ $(\lambda I+\bar{H})^{-1}$. Then for all $\varphi, \phi \in C_{c}^{\infty}(G)$ and $\operatorname{Re} \lambda$ large enough

$$
\begin{aligned}
\int_{0}^{\infty} d t e^{-\lambda t}\left(\psi, S_{t} \varphi\right) & =\left(\phi,(\lambda I+\bar{H})^{-1} \varphi\right) \\
& =\int_{G} d \hat{h} \int_{0}^{\infty} d t e^{-\lambda t} \overline{\psi(g)} K_{t}(g ; h) \varphi(h) \\
& =\int_{0}^{\infty} d t e^{-\lambda t}\left(\psi, T_{t} \varphi\right) .
\end{aligned}
$$

So $\left(\psi, S_{t} \varphi\right)=\left(\psi, T_{t} \varphi\right)$ and $S_{t}=T_{t}$. Therefore $S$ has a kernel and this kernel is $K$. It follows similarly that the semigroup on $L_{\hat{p}}$ has a kernel and that the kernel is $K$.

Since $K$ is independent of $p$ and $\hat{p}$, it follows automatically that $S$ is a consistent interpolating semigroup on the $L_{p^{-}}$, and $L_{\hat{p}^{-}}$, spaces. This finishes the proof of Statement I.

Step 4. All the foregoing arguments apply equally well to the formal adjoint $H^{\dagger}$ of $H$. Therefore the closures of $H^{\dagger}$ generate a consistent interpolating semigroup $S^{\dagger}$ on the $L_{p^{-}}$, and $L_{\hat{p}^{-}}$, spaces. But $\overline{H^{\dagger}}=H^{*}$ and hence $S^{\dagger}$ is dual to $S$. Therefore

$$
\begin{aligned}
\left(S_{t}^{\dagger} \phi, \varphi\right) & =\left(\phi, S_{t} \varphi\right) \\
& =\int_{G} d g \int_{G} d \hat{h} K_{t}(g ; h) \overline{\psi(g)} \varphi(h) \\
& =\int_{G} d g \int_{G} d \hat{h}\left(\Delta(g)^{-1} \Delta(h) K_{t}(h ; g)\right) \overline{\psi(h)} \varphi(g)
\end{aligned}
$$

for all $\varphi \in L_{p}$ and $\psi \in L_{q}$ with $p^{-1}+q^{-1}=1$. Consequently $K$ and $K^{\dagger}$ are related as in Statement IV of the theorem.

Step 5. Finally we consider the analyticity of the kernel $z \rightarrow K_{z}(g ; h)$ and the differentiability properties of $K_{t}$ and its derivatives $\partial_{t}^{n} K_{t}$. We begin with the kernel.

We have now established that the semigroup $S$ is holomorphic in a sector $\Delta_{\theta}$ with $\theta \geqq \theta_{C}$. Moreover, we have also shown that the kernel $K$ is defined in the sector $\Delta_{\theta_{C}}$. Now we want to prove that $z \mapsto K_{z}(g ; h)$ is analytic on $\Delta_{\theta_{C}}$ for all $g, h \in G$. Note that $L_{1} \otimes L_{1}$ is dense in $L_{1}(G \times G ; d g \times d g)$. There 
exists a unique strongly continuous holomorphic semigroup $T$ in $L_{1}(G \times G$; $d g \times d g)$, holomorphic in $\Delta_{0}$, such that $T_{z}(\varphi \otimes \psi)=\left(S_{z}^{\dagger} \varphi\right) \otimes \psi$ for all $\varphi, \phi \in L_{1}(G$; $d g)$ and $z \in \Delta_{\theta_{C}}$. Then $T^{*}$ is a holomorphic weakly* continuous holomorphic semigroup on $\mathcal{L}_{\infty}\left(=\mathcal{L}_{\infty}^{0}\right)$. Moreover, for all $z_{1}, z_{2} \in \Delta_{\theta C}$ one has $T_{z_{1}}^{*} K_{z_{2}}=K_{z_{1}+z_{2}}$. So $T_{z_{1}}^{*}$ acts strongly continuous on $K_{z_{2}}$ and hence the map $z \longrightarrow K_{z}$ from $\Delta_{0_{C}}$ into $\mathcal{L}_{\infty}$ is holomorphic, with the norm topology on $\mathcal{L}_{\infty}$. In particular, there exists $K_{z_{0}}^{\prime} \in \mathcal{L}_{\infty}$ such that

$$
\lim _{z \rightarrow z_{0}}\left\|\frac{K_{z}-K_{z_{0}}^{\prime}}{z-z_{0}}-K_{z_{0}}^{\prime}\right\|_{\perp_{\infty}}=0
$$

for all $z_{0} \in \Delta_{O_{C}}$. But $\left(z-z_{0}\right)^{-1}\left(K_{\varepsilon}-K_{z_{0}}\right)$ is a continuous function on $G \times G$, so $K_{z_{0}}^{\prime \prime}$ is also continuous and

$$
\lim _{z \rightarrow z_{0}} \frac{K_{6}(g ; h)-K_{z_{0}}(g ; h)}{z-z_{0}}=K_{z_{0}}^{\prime \prime}(g ; h)
$$

for all $g, h \in G$ and $z_{0} \in \Delta_{0_{C}}$. Hence $z \mapsto K_{z}(g ; h)$ is analytic in $\Delta_{O_{C}}$ for all $g$, $h \in G$.

Since $K_{t}^{(0)}(g ; h)=K_{t}^{g}\left(g h^{-1}\right)$ it follows from Proposition 2.3 that $K_{t}^{(0)}$, with $t>0$, is $(m-1)$-times pointwise differentiable in the first variable and that one has bounds

$$
\left|\left(t^{a} K_{t}^{(0)}\right)(g ; h)\right| \leqq a t^{-\left(D^{\prime}+|a|\right) / m} e^{\omega t} e^{-b\left(\left(\left|g h^{-1}\right|^{\prime}\right) m t-1,1 /(m-1)\right.}
$$

for all $g, h \in G$, all $t>0$ and all $\alpha$ with $\left|\alpha^{\prime}\right|<m$. The values of the parameters in these bounds depend only on the coefficients $c_{a}$ with $|\alpha|=m$ through the ellipticity constant and the norms $\left\|c_{a}\right\|_{\infty ; \ldots}^{\prime}$. Moreover, the same proposition gives bounds

$$
\left|L_{\iota}(g ; h)\right| \leqq a t^{-D^{\prime} / m} t^{-(n-1) / m} e^{\omega \iota} e^{-b\left(\left(\left|g h^{-1}\right|^{\prime}\right) m^{m-1}, 1 /(m-1)\right.}
$$

for all $g, h \in G$ and all $t>0$. These bounds depend, however, upon all the coefficients $c_{a}$ with $\alpha \in J_{m}\left(d^{\prime}\right)$ because the lower order coefficients enter in the definition of $L_{\iota}$. Nevertheless, it follows from Proposition 2.3 that only the derivatives of the principal coefficients enter the bounds and in fact only those derivatives of order less than or equal to $m$. Thus the $a, b$ and $\omega$ depend on the ellipticity constant and the parameter $\|C\|_{m}^{\prime}$ defined in Statement VI of the theorem. Hence it follows from the arguments of the appendix of [4] that $K_{t}$ is $(m-1)$-times differentiable in the first variable. It also follows from the argument used in the proof of Theorem 7.1 of [10] that one has bounds

$$
\left|\left(A^{a} K_{t}\right)(g ; h)\right| \leqq a t^{-\left(D^{\prime+}|a|\right) / m} e^{\omega t} e^{-b\left(\left(\left|g h^{-1}\right|^{\prime}\right) m t-1,1 /(m-1)\right.}
$$

for all $g, h \in G, t>0$ and $\alpha$ with $|\alpha|<m$ with the values of $a, b$ and $\omega$ dependent on the coefficients of $H$ only through the ellipticity constant and the parameter $\|C\|_{m}^{\prime}$. 
Next consider the case $|\alpha|=0$ and $n>0$. Since $z \mapsto K_{z}(g ; h)$ is analytic in the sector $\Delta_{\theta}$ the Cauchy representation gives

$$
K_{t}(g ; h)=(2 \pi i)^{-1} \int_{C_{r}(t)} d z \frac{K_{z}(g ; h)}{z-t}
$$

where the integral is over a circle $C_{r}(t)$ centred at $t$ with radius $r<t \sin \theta_{C}$. Hence

$$
\left(\partial_{t}^{n} K_{t}\right)(g ; h)=(2 \pi i)^{-1} n ! \int_{C_{r}(t)} d z \frac{K_{z}(g ; h)}{(z-t)^{n+1}}
$$

for all $n \in \boldsymbol{N}$. Therefore

$$
\left|\left(\partial_{t}^{n} K_{t}\right)(g ; h)\right| \leqq r^{-n} \sup \left\{\left|K_{z}(g ; h)\right|: z \in C_{r}(t)\right\}
$$

and setting $r=t \sin \varphi$ with $\varphi<\theta_{C}$ one obtains bounds

$$
\left|\left(\partial_{t}^{n} K_{t}\right)(g ; h)\right| \leqq a c^{n} n ! t^{-D^{\prime} / m-n} e^{\omega t} e^{-b\left(\left(\left.1 g h^{-1}\right|^{\prime}\right)^{m} t-1\right)^{1 /(m-1)}}
$$

for all $g, h \in G$ and all $t>0$ as a corollary of the bounds (17) on $\left|K_{z}\right|$.

The general bounds of Statement VI of Theorem 2.1 now follow from these estimates and the convolution semigroup property. The semigroup property gives the convolution relation

$$
K_{t}(g ; h)=\int_{G} d \hat{k} K_{s}(g ; k) K_{t-s}(k ; h)
$$

for all $s \in\langle 0, t\rangle$ and hence

$$
\left(A^{a} \partial_{t}^{n} K_{t}\right)(g ; h)=\int_{G} d \hat{k}\left(A^{\alpha} K_{s}\right)(g ; k)\left(\partial_{t}^{n} K_{t-s}\right)(k ; h)
$$

for all $s \in\langle 0, t\rangle$. Since each term has a 'Gaussian' bound, with an appropriate singularity, and the convolution of two Gaussians is a Gaussian, the required result follows by combination of the special cases with $s=t / 2$.

Next we prove Statement V. It follows from the convolution property of the $K_{t}$ and the adjoint relation of Statement IV that

$$
\begin{aligned}
K_{t}(g ; h) & =\int_{G} d \hat{k} K_{t / 2}(g ; k) K_{t / 2}(k ; h) \\
& =\int_{G} d \hat{k} K_{t / 2}(g ; k) \Delta(k)^{-1} \Delta(h) \overline{K_{t / 2}^{\dagger}(h ; k)} .
\end{aligned}
$$

Therefore the derivatives $A^{\alpha} K_{t}$ with $\alpha \in J_{m-1}\left(d^{\prime}\right)$ are $(m-1)$-times differentiable with respect to the second variable and

$$
\left(A^{\alpha} B^{\beta} K_{t}\right)(g ; h)=\int_{G} d \hat{k}\left(A^{\alpha} K_{t / 2}\right)(g ; k) \Delta(k)^{-1} \sum_{(\gamma, \delta) \in L b(\beta)}\left(A^{\gamma} \Delta\right)(h) \overline{\left(A^{\delta} K_{t / 2}^{+}\right)(h ; k)}
$$

where the $B$ denote left derivatives with respect to the second variable and the sum arises from the Leibniz formula for the left derivatives $A^{\beta}$ of the product $h \mapsto \Delta(h) K_{t / 2}^{\dagger}(h ; k)$. Similarly the $B^{\beta} K_{t}$ with $\beta \in J_{m-1}\left(d^{\prime}\right)$ are $(m-1)$ - 
times differentiable with respect to the first variable. Note that arguing as before one obtains bounds

$$
\left|\left(\alpha^{a} B^{\beta} K_{l}\right)(g ; h)\right| \leqq a t^{-\left(D^{\prime}+|a|+|\beta|\right) / m} e^{\omega l} e^{-b\left(\left(1 g h^{-\left.1\right|^{\prime}}\right) m l-1\right)^{1 /(m-1)}}
$$

but the use of the formal adjoint in this derivation means that the values of $a, b$ and $\omega$ depend now on the norms $\left\|c_{\alpha}\right\|_{\infty ;|\alpha|}^{\prime}$ for all $\alpha \in J_{m}\left(d^{\prime}\right)$. Hence these bounds are not useful for the subsequent discussion of operators with lower order coefficients which are not smooth.

Finally Statement VII follows directly from Statement VI. Let $\alpha \in J_{m-1}\left(d^{\prime}\right)$. Let $a, b, \omega>0$ be such that

$$
\left|\left(A^{a} K_{t}\right)(g ; h)\right| \leqq F_{t}\left(g h^{-1}\right)=a t^{-\left(D^{\prime}+|a|\right) / m} e^{\omega t} e^{-b\left(\left(\left|g h^{-1}\right|^{\prime}\right) m_{t-1}\right)^{1 /(m-1)}}
$$

for all $g, h \in G$ and $t>0$. Then

$$
\left\|A^{\alpha} S_{t} \varphi\right\|_{p} \leqq\left\|F_{t} *|\varphi|\right\|_{p} \leqq\left\|F_{t}\right\|_{1}\|\varphi\|_{p} \leqq a^{\prime} t^{-|a| / m} e^{\omega^{\prime} t}\|\varphi\|_{p}
$$

for all $p \in[1, \infty]$ and $\varphi \in L_{p}$. The argument on the $L_{\hat{p}}$-spaces is similar.

The kernel bounds of Theorem 2.1 immediately imply that the action of the semigroup $S$ is smooth. For example, it follows immediately that $S_{t} C_{0} \subseteq C_{0}$, for $t>0$, and in fact $S_{t} C_{0} \subseteq C_{0} \cap L_{\infty ; m-1}^{\prime}$. These conclusions are an immediate consequence of Statements V, VI and VII of the theorem. Moreover, it is also an easy consequence of the 'Gaussian' bounds on the semigroup kernel that $S_{t} L_{p} \subseteq C_{0}$ and $S_{t} L_{\hat{p}} \leqq C_{0}$ for all $p \in[1, \infty>$ and $t>0$. In the next sections we derive stronger versions of all these properties.

\section{§3. Regularity}

Let $C$ be an $m$-th order subcoercive form with $c_{\alpha} \in C_{b ; \infty}$ for all $\alpha \in J_{m}\left(d^{\prime}\right)$. The semigroup $S$ constructed in the previous section is holomorphic and hence

$$
S_{t} L_{p} \subseteq D^{\infty}(\bar{H})=\bigcap_{n \geqq 1} D\left(\bar{H}^{n}\right)
$$

for all $p \in[1, \infty]$ and all $t>0$. Now we utilize this observation to improve knowledge of the regularity, or smoothness, properties of the action of $S$ and the kernel $K$. Specifically we prove that the $C^{\infty}$-vectors for the left regular representation on the $L_{p^{-}}$, or $L_{\hat{p}^{-}}$, spaces are precisely the $C^{\infty}$-vectors $D^{\infty}(\bar{H})$ for the closure $\bar{H}$ of $H$. Therefore $S_{t} L_{p} \subseteq L_{p ; \infty}$ for all $p \in[1, \infty]$ and all $t>0$. The result for the $C^{\infty}$-vectors is derived from more detailed elliptic regularity properties of the domains $D\left(\bar{H}^{n}\right)$. For the case $p=2$ these latter regularity properties can be optimized by exploiting the unitarity of the left regular representation on $L_{2}$.

We use the same notation as in Section 2. Let $n \in \boldsymbol{N}$. For $g \in G$ and suf- 
ficiently large $\lambda>0$ define $R_{\lambda}^{(n), g}: G \backslash\{e\} \rightarrow C$ by

$$
R_{\Lambda}^{(n), g}(h)=(n-1) !^{-1} \int_{0}^{\infty} d t e^{-\dot{\lambda} t} t^{n-1} K_{t}^{g}(h) .
$$

Then $R_{\wedge}^{(n), g} \in L_{1}$ and

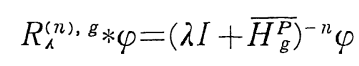

for all $\varphi \in L_{p}$. Moreover, for all $\beta \in J_{n m-1}\left(d^{\prime}\right)$ we have $R_{\lambda}^{(n), g} \in D\left(A^{3}\right)$, in the $L_{1}$-sense, and the function $h \mapsto R_{\lambda}^{(n), g}(h)$ from $G \backslash\{e\}$ into $C$ is infinitely differentiable. For a discussion of these facts, in the strongly elliptic case, see [20] Section III.6b. We wish to derive differentiability properties of the function $(g, h) \rightarrow\left(A^{\beta} R_{\lambda}^{(n), g}\right)(h)$ from $G \times G \backslash\{e\}$ into $C$. We denote the derivatives with respect to $g$ in the direction $a_{\imath}$ by $\partial_{\imath}$ and use multi-index notation, as before.

Lemma 3.1. Let $\alpha_{1} \in J_{n m-1}\left(d^{\prime}\right), k \in\{2, \cdots, n m+1\} \quad$ and $\alpha_{2}, \cdots, \alpha_{k} \in J_{n m}\left(d^{\prime}\right)$ where $\left|\alpha_{1}\right|+k-1 \leqq n m$.

I. There exist $c, \omega>0$ and for all $\lambda \geqq \omega$ there exists $b_{\lambda}, c_{\lambda}>0$ such that

$$
\begin{aligned}
& \int_{0}^{\infty} d t_{1} \cdots \int_{0}^{\infty} d t_{k} e^{-\lambda\left(t_{1}++t_{k}\right)}\left(t_{1} \cdots t_{k}\right)^{n-1}\left|\left(.1^{a_{1}} K_{t_{1}}^{g} g_{1} \cdots * A^{n k} K_{t_{k}}^{g k}\right)(h)\right|
\end{aligned}
$$

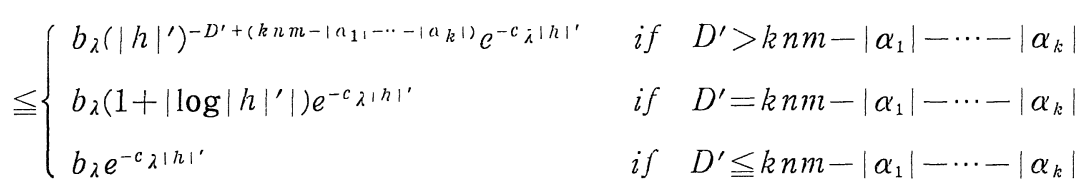

for all $h \subseteq G, h \neq e$ and all $g_{1}, \cdots, g_{k} \in G$ and

$$
\begin{aligned}
& \int_{G} d h \int_{0}^{\infty} d t_{1} \cdots \int_{0}^{\infty} d t_{k} e^{-\lambda\left(t_{1}++t_{k}\right)}\left(t_{1} \cdots t_{k}\right)^{n-1}\left|\left(--^{a_{1}} K_{t_{1}}^{g_{1 *}} \cdots * A^{a_{k}} K_{t_{k}}^{g_{k} k}\right)(h)\right| \\
& \leqq c \lambda^{-\left(k n l m-\left|a_{1}\right|--\left|a_{k}\right|\right) / m} \text {. }
\end{aligned}
$$

II. Fix $g_{1}, \cdots, g_{k} \in G$. For $\beta \in J_{\left|a_{1}\right|}\left(d^{\prime}\right)$ and $\lambda>\omega$, with $\omega>0$ sufficiently large depending only on $k$, define $F_{\beta}: G \backslash\{e\} \rightarrow C$ by

$$
F_{\beta}(h)=\int_{0}^{\infty} d t_{1} \cdots \int_{0}^{\infty} d t_{k} e^{-\lambda\left(t_{1}+\cdot+t_{k}\right)}\left(t_{1} \cdots t_{k}\right)^{n-1}\left(.1^{3} K_{t_{1} \psi_{-}}^{g_{1} 4^{a_{2}}} K_{t_{2}}^{g_{2} z_{*}} \cdots * 4^{a}{ }_{k} K_{t_{k}}^{g_{k}}\right)(h) .
$$

Then $F_{\beta} \in L_{1 ;\left|\alpha_{1}\right|-|\beta|}^{\prime}$ and $A^{\gamma} F_{\beta}=F_{\langle r, \beta\rangle}$ for all $\gamma \in J_{\left|\alpha_{1}\right|-|\beta|}\left(d^{\prime}\right)$. Moreover, the function $F_{\beta}$ is continuous.

III. There exists $\omega>0$ such that for all $\lambda>\omega$, all $g_{1}, \cdots, g_{k} \in G$ and $\varphi \in$ $C_{c}^{\infty}(G)$

$$
\begin{aligned}
& A^{a_{1}}\left(\lambda I+\overline{H_{g_{1}}^{P}}\right)^{-n} \cdots A^{a} k\left(\lambda I+\overline{H_{g_{k}}^{P}}\right)^{-n} \varphi \\
& =\left(\int_{0}^{\infty} d t_{1} \cdots \int_{0}^{\infty} d t_{k} e^{-\lambda\left(t_{1}+\cdot+t_{k}\right)}\left(t_{1} \cdots t_{k}\right)^{n-1} A^{a_{1}} K_{t_{1}}^{g_{1}} \cdots * A^{a_{k}} K_{t_{k}}^{g_{k}}\right) * \varphi
\end{aligned}
$$

IV. For all compact $K \subset G$ with $e \nsubseteq I$ we have 


$$
\begin{array}{r}
\lim _{g_{1}, \cdots, g_{k \rightarrow g}} \int_{0}^{\infty} d t_{1} \cdots \int_{0}^{\infty} d t_{k} e^{-\lambda\left(t_{1}+t_{k}\right)}\left(t_{1} \cdots t_{k}\right)^{n-1}\left(A^{a_{1}} K_{t_{1}}^{g_{1} * \cdots *} \cdot \mathcal{A}^{a_{k}} K_{t_{k}}^{g_{k}}\right)(h) \\
=\int_{0}^{\infty} d t_{1} \cdots \int_{0}^{\infty} d t_{k} e^{-\lambda\left(t_{1}++t_{k}\right)}\left(t_{1} \cdots t_{k}\right)^{n-1}\left(\mathcal{A}^{a_{1}} K_{t_{1}}^{g_{1}} \cdots \cdots *_{1} \mathcal{H}^{a_{k}} K_{t_{k}}^{g}\right)(h)
\end{array}
$$

for all $g \in G$, uniformly for $h \equiv K$.

Proof. Although the coefficients $c_{\alpha}$ are $C^{\infty}$ in this section, for this lemma we only need that $c_{a}$ is continuous in Statement IV.

It follows as in the proof of (12) in Lemma 2.2.I that there exist $a, b, \omega>0$ such that

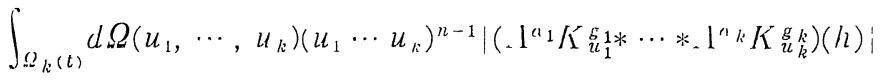

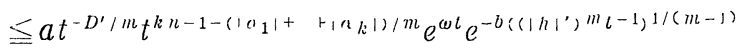

for all $t>0$ and $h \in G$. Hence

$$
\begin{aligned}
& \int_{0}^{\infty} d t_{1} \cdots \int_{0}^{\infty} d t_{k} e^{-\Lambda\left(l_{1}++t_{k}\right)}\left(t_{1} \cdots l_{h}\right)^{n-1}\left|\left(.1^{n} K_{l_{1}}^{g_{1}} \cdots \cdots * H^{n}{ }_{k} K_{t_{k}}^{g_{k}}\right)(h)\right| \\
& =k !^{-1 / 2} \int_{0}^{\infty} d l e^{-\lambda t} \int_{\Omega_{k}(l)} d \Omega\left(u_{1}, \cdots, u_{k}\right) \\
& \cdot\left(u_{1} \cdots u_{k}\right)^{n-1}\left|\left(\left.\cdot f^{a_{1}} K^{g} u_{1}^{g} * \cdots *_{-}\right|^{n k} K_{u_{k}}^{g}\right)(h)\right| \\
& \leqq k !^{-1 / 2} \int_{0}^{\infty} d t e^{-1 t} a t^{-D^{\prime \prime} n} t^{k n-1-\left(\left|n_{1}\right|^{+}+\left|n_{k}\right| 1 / m\right.} e^{\omega t} e^{\left.-b_{1}\left(\mid h_{1}\right)^{\prime}\right) m^{m} t-1,1 /(m-1)} \text {. }
\end{aligned}
$$

Now Statement I follows from [20], Theorem III.6.7, if $\omega$ is large enough. Moreover,

$$
\begin{aligned}
& \left.\int_{(i} d h \int_{0}^{\infty} d t_{1} \cdots \int_{0}^{\infty} d t_{k} e^{-\Lambda\left(t_{1}+\right.} t_{k}\right)\left(t_{1} \cdots t_{k}\right)^{n-1}\left|\left(A^{n} K_{t_{1}}^{g_{1}} \cdots * 1^{n k} K_{t_{k}}^{g_{k}}\right)(h)\right| \\
& \leqq k !^{-1 / 2} \int_{G} d h \int_{0}^{\infty} d t e^{-\wedge l} a t^{-D^{\prime} / m} t^{k n-1-\left(\left|a_{1}\right|++\left|a_{k}\right|\right) / m} e^{\omega t} e^{-b\left(\left.|l| h\right|^{\prime}\right) m_{t}-1,1 /(m-1)} \\
& \leqq a^{\prime} \int_{0}^{\infty} d t e^{\left(\omega^{\prime}-\Lambda\right) t} t^{k n-1-\left(\left|a_{1}\right|++\ln k \mid\right) / m} \\
& \leqq a^{\prime \prime} \lambda^{-\left(k n m-\left(\left|n_{1}\right|++\left|a_{k}\right|\right)\right) / m}
\end{aligned}
$$

if $\lambda \geqq 2 \omega^{\prime}$. This proves Statement I.

For the proof of Statement II it suffices to prove that for all $\beta \in J_{\left|a_{1}\right|-1}\left(d^{\prime}\right)$ and $i \in\left\{1, \cdots, d^{\prime}\right\}$ one has $F_{\beta} \in D\left(A_{2}\right)$, in the $L_{1}$-sense, and $A_{\imath} F_{\beta}=F_{\langle\imath, \beta\rangle}$. The proof is by duality. Let $\varphi \in D\left(. A_{2}\right)$, in the $L_{\infty}$-sense, and then by Fubini's theorem

$$
\begin{aligned}
& \left(A_{1} \varphi, F_{\beta}\right) \\
& \quad=\int_{0}^{\infty} d t_{1} \cdots \int_{0}^{\infty} d t_{k} e^{-\lambda\left(t_{1}+t_{k}\right)}\left(t_{1} \cdots t_{k}\right)^{n-1}\left(.1, \varphi, A^{\beta} K_{t_{1}}^{g_{1} *_{1}} A^{n_{2}} K_{t_{2}}^{g_{2}} \cdots *_{2} A^{a_{k}} K_{t_{k}}^{g_{k}}\right)
\end{aligned}
$$




$$
\begin{aligned}
& =-\int_{0}^{\infty} d t_{1} \cdots \int_{0}^{\infty} d t_{k} e^{-\lambda\left(t_{1}+\cdots+t_{k}\right)}\left(t_{1} \cdots t_{k}\right)^{n-1}\left(\varphi, A_{2} A^{\beta} K_{t_{1}}^{g_{1} * A^{a_{2}}} K_{t_{2}}^{g_{2}} \cdots \cdots A^{a_{k}} K_{t_{k}}^{g_{k}}\right) \\
& =-\left(\varphi, F_{\langle\imath, \beta\rangle}\right) .
\end{aligned}
$$

So $F_{\beta} \in D\left(A_{i}^{* *}\right)=D\left(A_{2}\right)$ and $A_{2} F_{\beta}=F_{\langle\imath, \beta\rangle}$. This proves Statement II, since the continuity follows as for the kernel.

Next we prove Statement III. First consider the case $k=2$. There exist $\alpha_{2}^{\prime}, \alpha_{2}^{\prime \prime} \in J_{n m-1}\left(d^{\prime}\right)$ such that $\alpha_{2}=\left\langle\alpha_{2}^{\prime}, \alpha_{2}^{\prime \prime}\right\rangle$. Then for $\varphi \in C_{c}^{\infty}(G)$ and large $\lambda$

$$
\begin{aligned}
& \left(\lambda I+\overline{H_{g_{1}}^{P}}\right)^{-n} A^{a_{2}}\left(\lambda I+\overline{H_{g_{2}}^{P}}\right)^{-n} \varphi \\
& =(-1)^{\mid a_{2}^{\prime}}{ }^{\prime}\left(A^{\left(a_{2}^{\prime}\right) *}\left(\lambda I+\left(H_{g_{1}}^{P}\right)^{*}\right)^{-n}\right)^{*} A^{a_{2}^{\prime \prime}}\left(\lambda I+\overline{H_{g_{2}}^{P}}\right)^{-n} \varphi \\
& =(-1)^{\mid a_{2}^{\prime}}{ }^{\prime}\left(\int_{0}^{\infty} d t_{1} e^{-\lambda t_{1}} t_{1}^{n-1}\left(\mathcal{A}^{\left(a_{2}^{\prime}\right) *} K_{t_{1}}^{g_{1}}{ }^{\dagger}\right)^{\sim}\right) *\left(\int_{0}^{\infty} d t_{2} e^{-\lambda t_{2}} t_{2}^{n-1} A^{\alpha_{2}^{\prime \prime}} K_{t_{2}}^{g_{2}}\right) * \varphi \\
& =\left(\int_{0}^{\infty} d t_{1} \int_{0}^{\infty} d t_{2} e^{-\lambda\left(t_{1}+t_{2}\right)}\left(t_{1} t_{2}\right)^{n-1} K_{t_{1}}^{g_{1}} * A^{a_{2}} K_{t_{2}}^{g_{2}}\right) * \varphi .
\end{aligned}
$$

So by Statement II we obtain

$$
\begin{aligned}
& A^{\alpha_{1}}\left(\lambda I+\overline{H_{g_{1}}^{P}}\right)^{-n} A^{\alpha_{2}}\left(\lambda I+\overline{H_{g_{2}}^{P}}\right)^{-n} \varphi \\
& =A^{\alpha_{1}}\left(\left(\int_{0}^{\infty} d t_{1} \int_{0}^{\infty} d t_{2} e^{-\lambda\left(t_{1}+t_{2}\right)}\left(t_{1} t_{2}\right)^{n-1} K_{t_{1}}^{g_{1} * A^{a_{2}} K_{t_{2}}^{g_{2}}}\right) * \varphi\right) \\
& =\left(A^{\alpha_{1}} \int_{0}^{\infty} d t_{1} \int_{0}^{\infty} d t_{2} e^{-\lambda\left(t_{1}+t_{2}\right)}\left(t_{1} t_{2}\right)^{n-1} K_{t_{1}}^{g_{1}} * A^{a_{2}} K_{t_{2}}^{g_{2}}\right) * \varphi \\
& =\left(\int_{0}^{\infty} d t_{1} \int_{0}^{\infty} d t_{2} e^{-\lambda\left(t_{1}+t_{2}\right)}\left(t_{1} t_{2}\right)^{n-1} A^{a_{1}} K_{t_{1}}^{g_{1}} * A^{a_{2}} K_{t_{2}}^{g_{2}}\right) * \varphi .
\end{aligned}
$$

This proves (23) if $k=2$.

Now suppose that $(23)$ is valid for some $k \in\{2, \cdots, n m\}$. Let $\alpha_{2}, \cdots, \alpha_{k+1} \in$ $J_{n m}\left(d^{\prime}\right)$. Let $\alpha_{2}^{\prime}, \alpha_{2}^{\prime \prime} \in J_{n m-1}\left(d^{\prime}\right)$ be such that $\alpha_{2}=\left\langle\alpha_{2}^{\prime}, \alpha_{2}^{\prime \prime}\right\rangle$. Then it follows from the induction hypothesis that (23) is valid if $\left|\alpha_{1}\right|=0$ by a similar argument to the above and then again by Statement II for general $\alpha_{1}$.

Finally, the proof of Statement IV follows from (14), by the reasoning used in the proof of Lemma 2.2.III.

The next result is of the same type as Proposition 2.3.

Proposition 3.2. Suppose $c_{\alpha} \in C_{b ; \infty}$ for all $\alpha \in J_{m}\left(d^{\prime}\right)$.

I. There exists $\omega>0$ such that for all $\lambda \geqq \omega$, all $k \in\{0, \cdots, n m-1\}$ and all $l \in\{0, \cdots, n m\}$ such that $k+l \leqq n m$ the function $(g, h) \mapsto R_{\wedge}^{(n), g}(h)$ from $G \times G \backslash\{e\}$ is k-times pointwise left-differentiable in $g$ and l-times left-differentiable in $h$, in any order, the derivatives are (jointly) continuous and the derivatives with respect to $g$ commute with those with respect to $h$.

II. There exist $c, \omega>0$ such that for all $\lambda \geqq \omega$, all $g \in G$, all $\beta \in J_{n m-1}\left(d^{\prime}\right)$ and all $\gamma \in J_{n m-1 \beta \mid}\left(d^{\prime}\right)$ such that $|\beta|+|\gamma| \leqq n m$ one has estimates 


$$
\left\|A^{3} \partial^{\gamma} R_{\Lambda}^{(n), g}\right\|_{1} \leqq c \lambda^{-(n m-|\beta|) / m}
$$

and the values of $c$ and $\omega$ depend continuously on the ellipticity constant $\mu$ and

$$
\|C\|_{n m}^{\prime}=\sum_{a ;|a|=m}\left\|c_{\alpha}\right\|_{\infty ; n m}^{\prime}+\sum_{a \in J} \sum_{m-1\left(d^{\prime}\right)}\left\|c_{\alpha}\right\|_{\infty} .
$$

Proof. For $k \in N, \alpha \in J_{k m}\left(d^{\prime}\right)$ and $g \in G$ define $c_{\alpha, k}(g) \in C$ in the most obvious way such that

$$
\left(H_{g}^{P}\right)^{k}=\sum_{\alpha ;|\alpha|=k m} c_{\alpha, k}(g) A^{a} .
$$

So this equality is even valid in the tensor algebra over $\mathrm{g}$. The $c_{a, k}$ are homogeneous polynomials of order $k$ in the $c_{\beta}$ with $|\boldsymbol{\beta}|=m$.

Fix $\beta \in J_{n m-1}\left(d^{\prime}\right)$. Let $g_{1}, g_{2} \in G, \lambda>0$ large and $\varphi \in C_{c}^{\infty}(G)$. Then

$$
\begin{aligned}
& \left(\left(\int_{0}^{\infty} d t e^{-\lambda t} t^{n-1} A^{3} K_{t}^{g_{1}}\right)-\left(\int_{0}^{\infty} d t e^{-\lambda t t^{n-1} A^{\beta} K_{t}^{g_{2}}}\right)\right) * \varphi \\
& \quad=A^{\beta}\left(\lambda I+\overline{H_{g_{1}}^{P}}\right)^{-n} \varphi-A^{\beta}\left(\lambda I+\overline{H_{g_{2}}^{P}}\right)^{-n} \varphi \\
& \quad=A^{\beta}\left(\lambda I+\overline{H_{g_{1}}^{P}}\right)^{-n}\left(\left(\lambda I+\overline{H_{g_{2}}^{P}}\right)^{n}-\left(\lambda I+\overline{H_{g_{1}}^{P}}\right)^{n}\right)\left(\lambda I+\overline{{H_{g_{2}}^{P}}^{2}}\right)^{-n} \varphi \\
& \quad=\sum_{j=1}^{n}\left(\begin{array}{c}
n \\
j
\end{array}\right) \lambda^{n-\jmath} \sum_{a ;|a|=j m}\left(c_{\alpha, j}\left(g_{2}\right)-c_{a, j}\left(g_{1}\right)\right) A^{\beta}\left(\lambda I+\overline{H_{g_{1}}^{P}}\right)^{-n} \cdot A^{n}\left(\lambda I+\overline{H_{g_{2}}^{P}}\right)^{-n} \varphi .
\end{aligned}
$$

Hence by Lemma 3.1.III

$$
\begin{gathered}
\left(\int_{0}^{\infty} d t e^{-\lambda t} t^{n-1} A^{\beta} K_{t}^{g_{1}}\right)-\left(\int_{0}^{\infty} d t e^{-\lambda t} t^{n-1} A^{\beta} K_{t}^{g_{2}}\right) \\
=\sum_{j=1}^{n}\left(\begin{array}{c}
n \\
j
\end{array}\right) \lambda^{n-j} \sum_{\alpha ;|\alpha|=j n}\left(c_{\alpha, j}\left(g_{2}\right)-c_{\alpha, j}\left(g_{1}\right)\right) \\
\quad \cdot \int_{0}^{\infty} d t_{1} \int_{0}^{\infty} d t_{2} e^{-\lambda\left(t_{1}+t_{2}\right)}\left(t_{1} t_{2}\right)^{n-1} \cdot A^{\beta} K_{t_{1}}^{g_{1}} * A^{a} K_{t_{2}}^{g_{2}},
\end{gathered}
$$

in the $L_{1}$-sense, but then also pointwise on $G \backslash\{e\}$ by continuity (see Lemma 3.1.II).

We shall prove that for all $\gamma \in J_{n m-|\beta|}\left(d^{\prime}\right),|\gamma| \neq 0$, all $k \in\{2, \cdots,|\gamma|+1\}$ and all $\alpha_{2}, \cdots, \alpha_{k} \in J_{n m}\left(d^{\prime}\right)$ there exist $c_{a_{2}, \cdots, \alpha_{k}}^{\gamma, k} \in C(\boldsymbol{R} \times G)$, polynomials in $\lambda$ and the $A^{\delta} c_{\alpha}$ with $\delta \in J_{|r|}\left(d^{\prime}\right)$ and $|\alpha|=m$, such that

$$
\begin{gathered}
\left(\partial^{r} A^{\beta} R_{\lambda}^{(n), g}\right)(h) \\
=\sum_{k=2}^{|\gamma|+1} \sum_{\alpha_{2}, \cdots, \alpha_{k} \in J_{n m}\left(d^{\prime}\right)} c_{a_{2}, \cdots, \alpha_{k}}^{\gamma, k}(\lambda, g) \int_{0}^{\infty} d t_{1} \cdots \int_{0}^{\infty} d t_{k} e^{-\lambda\left(t_{1}++t_{k}\right)}\left(t_{1} \cdots t_{k}\right)^{n-1} \\
\cdot\left(A^{\beta} K_{t_{1}}^{g} * A^{a_{2}} K_{t_{2}}^{g} * \cdots * A^{a_{k}} K_{t_{k}}^{g}\right)(h)
\end{gathered}
$$

for all large $\lambda>0$ and $g, h \in G$ with $h \neq 0$. Moreover, $c_{\alpha_{2}, \cdots, a_{k}}^{\gamma, k}$ is homogeneous in $\lambda$ of degree $(k-1) n-\left(\left|\alpha_{2}\right|+\cdots+\left|\alpha_{k}\right|\right) / m$. The proof is by induction on $|\gamma|$ and the case $|\gamma|=1$ follows from (24) and Lemma 3.1.IV.

Now let $\gamma \in J_{n m-1 \beta 1-1}\left(d^{\prime}\right)$ and suppose (25) is valid. Let $i \in\left\{1, \cdots, d^{\prime}\right\}$. Then 
for all large $\lambda>0$ and $g \in G$, with $g_{s}=\exp \left(-s a_{\imath}\right) g$ for all $s \in \boldsymbol{R}$ one has for $s \neq 0$ and $\varphi \in C_{c}^{\infty}(G)$, with the notation $\alpha_{1}=\beta$ one obtains by Lemma 3.1.III

$$
\begin{aligned}
& \left(s^{-1}\left(\partial^{i}, A^{\beta} R_{\lambda}^{(n), g s}-\partial^{r} \cdot A^{\beta} R_{\lambda}^{(n), g}\right)\right) * \varphi
\end{aligned}
$$

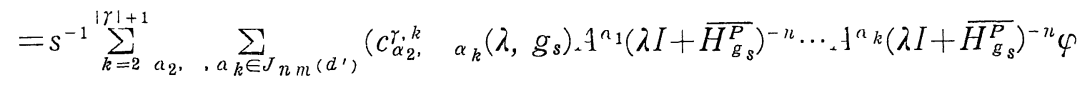

$$
\begin{aligned}
& \left.-c_{a_{2}, a_{k}}^{\gamma, k}(\lambda, g)_{A^{2}} a_{1}\left(\lambda I+\overline{H_{g}^{P}}\right)^{-n} \cdots f^{a_{k}}\left(\lambda I+\overline{H_{g}^{\bar{P}}}\right)^{-n} \varphi\right)
\end{aligned}
$$

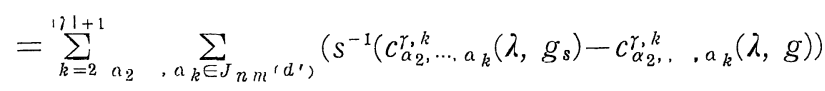

$$
\begin{aligned}
& \cdot A^{a_{1}}\left(\lambda I+\overline{H_{g_{s}}^{P}}\right)^{-n} \cdots \cdot \mathcal{H}^{\alpha{ }_{k}}\left(\lambda I+\overline{H_{g_{s}}^{P}}\right)^{-n} \varphi \\
& +\sum_{l=1}^{k} c_{a_{2},}^{\gamma_{2}, k} \cdot a_{k}(\lambda, g) \cdot 4^{a_{1}}\left(\lambda I+\overline{H_{g_{s}}^{P}}\right)^{-n} \cdots s^{-1}\left(A^{a_{l}}\left(\lambda I+\overline{H_{g_{s}}^{P}}\right)^{-n}-1^{a_{1}}\left(\lambda I+\overline{H_{g}^{P}}\right)^{-n}\right) \\
& \cdots, 1^{a}{ }^{k}\left(\lambda I+{\overline{H_{g}^{P}}}^{-n} \varphi\right) \\
& =\sum_{k=2}^{|\gamma|+1} \sum_{a_{2}, \ldots} \sum_{k \in J n l\left(d^{\prime}\right)}\left(s^{-1}\left(c_{a_{2}, \cdots, \alpha_{k}}^{\gamma, k}\left(\lambda, g_{s}\right)-c_{\alpha_{2}, \alpha_{k}}^{\gamma, k}(\lambda, g)\right)\right. \\
& \cdot A^{a_{1}}\left(\lambda I+\overline{H_{g_{s}}^{P}}\right)^{-n} \cdots A^{\alpha_{k}}\left(\lambda I+\overline{H_{g_{s}}^{P}}\right)^{-n} \varphi \\
& +\sum_{l=1}^{k} \sum_{j=1}^{n}\left(\begin{array}{c}
n \\
j
\end{array}\right) \lambda^{n-\jmath} \sum_{a ;|a|=j m} c_{a_{2},}^{\gamma, k} \quad a_{k}(\lambda, g) S^{-1}\left(c_{a, j}\left(g_{s}\right)-c_{\alpha, j}(g)\right)_{-} \mathcal{H}^{a_{1}}\left(\lambda I+\overline{H_{g_{0}}^{P}}\right)^{-n} \\
& \left.\cdots A^{\alpha} l\left(\lambda I+\overline{H_{g}^{P}}\right)^{-n} \cdot A^{a}\left(\lambda I+\overline{H_{g}^{P}}\right)^{-n} \cdots \cdot 4^{a}{ }^{k}\left(\lambda I+\overline{H_{g}^{P}}\right)^{-n} \varphi\right) .
\end{aligned}
$$

Using Lemma 3.1.III again one obtains an equation of the form (24), valid first in the $L_{1}$-sense, but by continuity also pointwise on $G \backslash\{e\}$. Taking the limit $s \rightarrow 0$ one readily establishes equality (25), using Lemma 3.1.IV again. It follows as in the proof of Proposition 2.3 that the derivatives with respect to $g$ and $h$ commute, if one uses Lemma 3.1.II and the lemma of Du Bois-Reymond.

The proof of Statement II is easy.

Before we can prove the main theorem of this section we need one more technical lemma. The next lemma provides a condition which ensures that pointwise differentiability implies $L_{1}$-differentiability.

Lemma 3.3. Let $h \in G, F \in C(G \backslash\{h\}) \cap L_{1}$ and suppose that $F$ is pointwise left-differentiable on $G \backslash\{h\}$ in the directions $a_{1}, \cdots . a_{d}$. with pointwise derivatives $\mathcal{A}_{l}^{(p)} F \in C(G \backslash\{h\}) \cap L_{1}$ for all $i \in\left\{1, \cdots, d^{\prime}\right\}$. Moreover, suppose that

$$
\lim _{s \rightarrow 0} s^{-1} \int_{B_{t}^{\prime}(h)} d g|F(g)|=0
$$

where $B_{s}^{\prime}(h)=\left\{g \in G:\left|g h^{-1}\right|<s\right\}$. Then $F \in L_{1 ; 1}^{\prime}$ and $A_{2} F=A_{2}^{(p)} F$ for all $i \in$ $\left\{1, \cdots, d^{\prime}\right\}$.

Proof. Let $F_{e}: G \backslash\{e\} \rightarrow C$ be delined by $F_{c}(g)=F(g / 2)$. Since 


$$
\int_{B_{s}^{\prime}} d g\left|F_{\rho}(g)\right|=\Delta(h)^{-1} \int_{B_{s}^{\prime}(h)} d g|F(g)|
$$

and left derivatives commute with right translations, we may as well assume that $h=e$. We shall prove that $\left(A_{\imath} \psi, F\right)=-\left(\psi, \perp_{l}^{(p)} F\right)$ for all $\phi \in C_{c}^{\infty}(G)$. It then follows by density ([2] Corollary 3.1.7) and Lemma 2.4 that $\left(A_{2} \psi, F\right)=-$ $\left(\psi, A_{i}^{(p)} F\right)$ for all $\phi \in D\left(A_{1}\right)$, in the $L_{\infty}$-sense, and hence $F \in D\left(A_{2}\right)$, in the $L_{1}$ sense, and $A_{2} F=A_{2}^{(p)} F$.

First suppose that $\phi \in C_{c}^{\infty}(G)$ and $e \notin \operatorname{supp} \psi$. Then it follows easily from the mean value theorem that $\left(A_{2} \psi, F\right)=-\left(\psi, A_{\imath}^{(p)} F\right)$.

Secondly, suppose there exist $a, b>0, \chi_{1}, \chi_{2}, \cdots \in C_{c}^{\infty}(G)$ such that $0 \leqq \chi_{n} \leqq 1$, | $\chi_{n} \|_{\infty ; 1}^{\prime} \leqq a n$, supp $\chi_{n} \subseteq B_{b n}^{\prime}{ }^{-1}$ and $\left\{g \in G: \chi_{n}(g)=1\right\}$ is a neighbourhood of $e$ for all $n \in N$. Using the existence of these functions it follows that for all $\phi \in$ $C_{c}^{\infty}(G)$ the function $\phi\left(1-\chi_{n}\right) \in C_{c}^{\infty}(G)$ and $e \notin \operatorname{supp} \phi\left(1-\chi_{n}\right)$. So

$$
\left(\Lambda_{\imath} \psi-\left(A_{2} \psi\right) \chi_{n}-\psi A_{1} \chi_{n}, F\right)=\left(1_{1}\left(\psi\left(1-\chi_{n}\right)\right), F\right)=-\left(\psi\left(1-\chi_{n}\right), \Lambda_{1}^{(p)} F\right) .
$$

Taking the limit $n \rightarrow \infty$ one obtains

But

$$
\left(A_{1} \psi, F\right)-\lim _{n \cdot \infty}\left(\psi A_{\imath} \chi_{n}, F\right)=-\left(\psi, A_{i}^{(p)} F\right) .
$$

$$
\left|\left(\psi A_{\imath} \chi_{n}, F\right)\right| \leqq\|\psi\|_{\infty}\left\|A_{\imath} \chi_{n}\right\|_{\infty} \int_{B_{b n-1}^{\prime}} d g|F(g)| \leqq a\|\psi\|_{\infty} n \int_{B_{b n-1}^{\prime}} d g|F(g)|
$$

So $\lim _{n \rightarrow \infty}\left(\phi A_{2} \chi_{n}, F\right)=0$ and $\left(A_{2} \psi, F\right)=-\left(\psi, A_{2}^{(p)} F\right)$.

It remains to establish the existence of the functions $\chi_{1}, \chi_{2}, \cdots$. For $n \in N$ let $g_{n}$ be the linear span of all commutators of $a_{1}, \cdots, a_{d^{\prime}}$ of order less than or equal to $n$. Then $g_{1} \subset g_{2} \subset \cdots \subset g_{r}=g$. Let $d_{1}=d^{\prime}, a_{1 j}=a_{j}$ and inductively for $i \in\{2, \cdots, r\}$ let $a_{\imath 1}, \cdots, a_{\iota d_{i}} \in g_{\imath}$ be such that $a_{11}, \cdots, a_{1 d_{1}}, \cdots, a_{\imath 1}, \cdots, a_{\imath d_{i}}$ is a basis for $g_{\imath}$. The function $\Phi\left(\left(\xi_{\imath j}\right)\right)=\exp \left(\sum_{\imath=1}^{r} \sum_{\jmath=1}^{d} \xi_{\imath j} a_{\imath \jmath}\right)$ from $\boldsymbol{R}^{d_{1}} \times \cdots \times \boldsymbol{R}^{d_{r}}$ into $G$ is an analytic diffeomorphism in a neighbourhood of 0 . We may assume that it is a diffeomorphism on at least $V=\left\{\left(\xi_{\imath \jmath}\right):\left|\xi_{\imath \jmath}\right|<2\right.$ for all $i$ and $\left.j\right\}$. The function

$$
\Psi\left(\left(\xi_{\imath j}\right),\left(\eta_{\imath j}\right)\right)=\Phi^{-1}\left(\exp \left(\sum_{i=1}^{r} \sum_{j=1}^{d_{2}} \xi_{\imath \jmath} a_{\imath j}\right) \exp \left(\sum_{i=1}^{r} \sum_{j=1}^{d_{2}} \eta_{\imath \jmath} a_{\imath j}\right)\right)
$$

is analytic on a neighbourhood of $(0,0)$ and again we may assume that this neighbourhood contains $V^{2}$. We may also assume that the series in the Campbell-Baker-Hausdorfi formula is absolutely convergent on $V$. Now let $f: \boldsymbol{R}^{d_{1}}$ $\times \cdots \times \boldsymbol{R}^{d_{r} \rightarrow \boldsymbol{R}}$ be an infinitely differentiable function such that $0 \leqq f \leqq 1$, supp $f$ $\subseteq\left\{\left(\xi_{\imath j}\right):\left|\xi_{\imath \jmath}\right| \leqq 1\right\}$ and $f\left(\left(\xi_{\imath,}\right)\right)=1$ if $\left|\xi_{\imath \jmath}\right| \leqq 2^{-1}$ for all $i$ and $j$. For $n \in \boldsymbol{N}$ define $\chi_{n}: G \rightarrow \boldsymbol{R}$ by

$$
\chi_{n}(g)=\left\{\begin{array}{lll}
f\left(\left(n^{\imath} \xi_{\imath \jmath}\right)\right) & \text { if } & g=\Phi\left(\left(\xi_{\imath \jmath}\right)\right) \in \Phi(V), \\
0 & \text { if } & g \notin \Phi(V) .
\end{array}\right.
$$


Let $g \in G$ and suppose that $\chi_{n}(g) \neq 0$. Then there exists $\left(\xi_{i j}\right) \in V$ such that $g=$ $\exp \left(\sum_{i=1}^{r} \sum_{j=1}^{d_{\imath}} \xi_{i j} a_{i j}\right)$ and $\left|\xi_{i j}\right| \leqq n^{-i}$ for all $i$ and $j$. Let $\tilde{X}_{i j}$ be the left invariant vector field on $G$ which corresponds to $a_{i j} \in g$. If we take the path $\gamma(t)=\exp \left(\sum_{i=1}^{r} \sum_{j=1}^{d i} t \xi_{i j} a_{i j}\right)$ from $e$ to $g$ then $\dot{\gamma}(t)=\left.\sum_{i=1}^{r=1} \sum_{j=1}^{d i} \xi_{i j} \tilde{X}_{i j}\right|_{\gamma(t)}$, so by [17], Theorem 4, there exists $b>0$ such that $|g|^{\prime} \leqq b n^{-1}$. Hence supp $\chi_{n} \leqq B_{b n-1}^{\prime}$. Finally we prove that $\sup \left\{n^{-1}\left\|\chi_{n}\right\|_{\infty ; 1}^{\prime}: n \in \boldsymbol{N}\right\}<\infty$. Let $k \in\left\{1, \cdots, d^{\prime}\right\}, n \in \mathbb{N}$ and $g=\Phi\left(\left(\xi_{i j}\right)\right) \in \Phi(V)$. Let $\Psi=\sum_{N=1}^{\infty} \Psi_{N}$ be the series expansion of $\Psi$ in the Campbell-Baker-Hausdorff formula, with each $\Psi_{N}$ homogeneous of order $N$. Then

$$
\begin{aligned}
\left(A_{k} \chi_{n}\right)(g) & =\left.\frac{d}{d t} \chi_{n}\left(\exp \left(-t a_{1 k}\right) \exp \left(\sum_{i=1}^{r} \sum_{j=1}^{d_{2}} \xi_{i j} a_{i j}\right)\right)\right|_{t=0} \\
& =\left.\frac{d}{d t} \chi_{n}\left(\Phi\left(\sum_{N=1}^{\infty} \Psi_{N}\left(-t \delta_{1 k},\left(\xi_{i j}\right)\right)\right)\right)\right|_{t=0} \\
& =\left.\frac{d}{d t} f\left(\sum_{N=1}^{\infty} \sum_{u=1}^{r} \sum_{v=1}^{d u} n^{u}\left[\Psi_{N}\left(-t \delta_{1 k},\left(\xi_{i j}\right)\right)\right]_{u v}\right)\right|_{t=0} \\
& =\left.\sum_{u=1}^{r} \sum_{v=1}^{d u}\left(D_{u v} f\right)\left(\left(n^{i} \xi_{i j}\right)\right) n^{u} \sum_{N=1}^{\infty} \frac{d}{d t}\left[\Psi_{N}\left(-t \delta_{1 k},\left(\xi_{\imath \jmath}\right)\right)\right]_{u v}\right|_{t=0} .
\end{aligned}
$$

Now suppose that $\left(A_{k} \chi_{n}\right)(g) \neq 0$. Then $\left|\xi_{\imath j}\right| \leqq n^{-\imath}$ for all $i$ and $j$. Moreover, $\Psi_{N}$ is a homogeneous polynomial of order $N$, so by the Campbell-Baker-Hausdorff

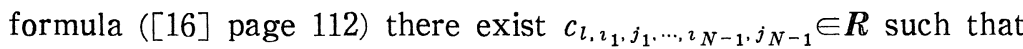

$$
\sum_{N=1}^{\infty} \sum_{l=0}^{N-1} \sum_{i_{1}, \ldots, i_{N-1}=1}^{r} \sum_{j_{1}=1}^{d_{i_{1}}} \ldots \sum_{j_{N-1}=1}^{d_{i_{N}-1}}\left|c_{l, i_{1}, j_{1}, \ldots, i_{N-1}, j_{N-1}}\right|<\infty
$$

and

$$
\begin{aligned}
& \left.\sum_{u=1}^{r} \sum_{v=1}^{d u} \frac{d}{d t}\left[\Psi_{N}\left(-t \delta_{1 k},\left(\xi_{\imath j}\right)\right)\right]_{u v} a_{u v}\right|_{t=0}
\end{aligned}
$$

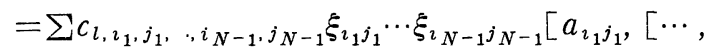

$$
\begin{aligned}
& \left.\left.\cdot\left[a_{i_{l} j_{l}},\left[a_{1 k},\left[a_{\imath_{l+1} j_{l+1}}, \cdots,\left[a_{\imath_{N-2} j_{N-2}}, a_{\imath_{N-1} j_{N-1}}\right] \cdots\right]\right]\right] \cdots\right]\right] \text {, }
\end{aligned}
$$

where the sum is over all $l \in\{0, \cdots, N-1\}, i_{1}, \cdots, i_{N-1} \in\{1, \cdots, r\}$ and $j_{w} \in$ $\left\{1, \cdots, d_{i_{w}}\right\}$ for all $w \in\{1, \cdots, N-1\}$. Now the commutator is an element of

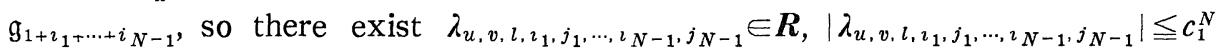
for some $c_{1}>0$ such that

$$
\begin{aligned}
{\left[a_{\imath_{1} j_{1}},\left[\cdots,\left[a_{\imath_{l} j_{l}},\left[a_{1 k},\left[a_{i_{l+1} j_{l+1}}, \cdots,\left[a_{i_{N-2} j_{N-2}}, a_{i_{N-1} j_{N-1}}\right] \cdots\right]\right]\right] \cdots\right]\right] } \\
=\sum_{u=1}^{\bar{r}} \sum_{v=1}^{i} \lambda_{u, v, l, \imath_{1}, j_{1}, \cdots, \imath_{N-1}, j_{N-1}} a_{u v}
\end{aligned}
$$

with $\bar{r}=r \wedge\left(1+i_{1}+\cdots+i_{N-1}\right)$. Then 


$$
\begin{aligned}
& \left|\left(A_{k} \chi_{n}\right)(g)\right| \\
& \leqq \sum_{u=1}^{r} \sum_{v=1}^{d u}\left\|D_{u v} f\right\|_{\infty} n^{u} \sum_{N=1}^{\infty} \sum_{\substack{l=0 \\
j_{1}}} \sum_{\begin{array}{c}
i_{1}, \cdots, i_{N-1} \\
j_{1}, \cdots, j_{N-1} \\
1+i_{1}+\cdots, i_{N-1} \geqq u
\end{array}}\left|c_{l, \imath_{1}, j_{1}, \cdots, \imath_{N-1} j_{N-1}}\right| \\
& \cdot\left|\xi_{i_{1} j_{1}}\right| \cdots\left|\xi_{i_{N-1} j_{N-1}}\right|\left|\lambda_{u, v, l, l_{1}, j_{1}, \cdots, l_{N-1}, j_{N-1}}\right| \\
& \leqq \sum_{u=1}^{r} \sum_{v=1}^{d u}\left\|D_{u v} f\right\|_{\infty} n^{u} \sum_{N=1}^{\infty} \sum_{l=0}^{N-1} \sum_{\begin{array}{c}
i_{1}, \cdots, i_{N-1} \\
j_{1}, \cdots, j N-1 \\
1+i_{1}+\cdots+i_{N-1}
\end{array}}\left|c_{l, i_{1}, j_{1}, \ldots, l_{N-1}, j_{N-1}}\right| n^{-\left(i_{1}+\cdots+i_{N-1}\right)} c_{1}^{N} .
\end{aligned}
$$

Now write the last sum as a sum of two contributions, one with $N \leqq r-1$ and one with $N \geqq r$. The first is estimated as follows

$$
\begin{aligned}
& \sum_{u=1}^{r} \sum_{v=0}^{d u}\left\|D_{u v} f\right\|_{\infty} n^{u} \sum_{N=1}^{r-1} \sum_{l=0}^{N-1} \sum_{\begin{array}{c}
i_{1}, \cdots, i_{N-1} \\
j_{1}, \cdots, j N-1 \\
1+i_{1}+\cdots+i_{N-1} \geqq u
\end{array}}\left|c_{l, i_{1}, j_{1}, \cdots, i_{N-1}, j_{N-1}}\right| n^{-\left(i_{1}+\cdots+i_{N-1}\right)} c_{1}^{N}
\end{aligned}
$$

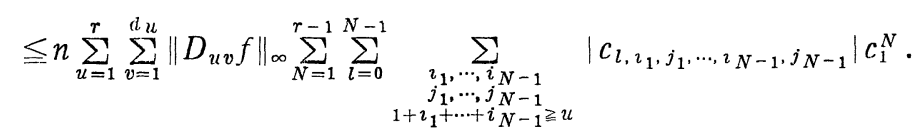

In order to estimate the second one first note that $i_{1}+\cdots+i_{N-1} \geqq N-1$. So

$$
\begin{gathered}
\sum_{u=1}^{r} \sum_{v=1}^{d_{u}}\left\|D_{u v} f\right\|_{\infty} n^{u} \sum_{N=r}^{\infty} \sum_{l=0}^{N-1} \sum_{\substack{i_{1}, \cdots, i_{N-1} \\
j_{1}, \cdots, j_{N-1}}}\left|c_{l, \nu_{1}, j_{1}, \cdots, j_{N-1}, j_{N-1} \mid}\right| n^{-\left(i_{1}+\cdots+i_{N-1}\right)} c_{1}^{N} \\
\leqq n \sum_{u=1}^{r} \sum_{v=1}^{d u}\left\|D_{u v} f\right\|_{\infty} c_{1}^{r} \sum_{N=r}^{\infty} \sum_{\substack{l=0 \\
j_{1}}}^{N-1} \sum_{\substack{i_{1}, \cdots, i_{N-1} \\
j_{1}, \cdots, j_{N-1}}}\left|c_{l, i_{1}, j_{1}, \cdots, i_{N-1}, j_{N-1}}\right|\left(c_{1} n^{-1}\right)^{N-r} .
\end{gathered}
$$

Since

$$
\sum_{N=r}^{\infty} \sum_{l=0}^{N-1} \sum_{\substack{i_{1}, \cdots, i_{N-1} \\ j_{1}, \ldots, j_{N-1}}}\left|c_{l, \imath_{1}, j_{1}, \cdots, i_{N-1}, j_{N-1}}\right|\left(c_{1} n^{-1}\right)^{N-r}<\infty
$$

for all $n \geqq c_{1}$, the lemma is proved.

Theorem 3.4. Let $C$ be an $m$-th order subcoercive form with coefficients $c_{\alpha} \in C_{b ; \infty}$ and let $H=d L(C)$. Let $p \in[1, \infty]$. Then

I. $D^{\infty}(\bar{H})=L_{p ; \infty}$.

II. For all $n \in \boldsymbol{N}$ one has $D\left(\bar{H}^{n}\right) \cong L_{p ; n m-1}^{\prime}$ and for all $k \in\{1, \cdots, n m-1\}$ there exists $c>0$ such that

$$
\|\varphi\|_{p ; k}^{\prime} \leqq \varepsilon^{n m-k}\left\|_{i} \bar{H}^{n} \varphi\right\|_{p}+c \varepsilon^{-k}\|\varphi\|_{p}
$$

for all $\varepsilon \in\langle 0,1]$ and $\varphi \in D\left(\bar{H}^{n}\right)$. The constant $c$ depends continuously on the ellipticity constant $\mu,\|C\|_{n m}^{\prime}$ and the $\left\|c_{\alpha}\right\|_{\infty ;(n-1) m}^{\prime}$ with $\alpha \in J_{m-1}\left(d^{\prime}\right)$.

III. If $k \in \boldsymbol{N}$ then $S_{t} L_{p} \cong L_{p ; k}^{\prime}$ and there exists $c_{k}>0$ such that

$$
\left\|S_{t} \varphi\right\|_{p ; k}^{\prime} \leqq c_{k} t^{-k / m}\|\varphi\|_{p}
$$


for all $\varphi \in L_{p}$ and $t \in\langle 0,1]$. The constant $c_{k}$ depends continuously on the ellipticity constant $\mu,\|C\|_{n m}^{\prime}$ and the $\left\|_{1}^{\prime} c_{\alpha}\right\|_{\infty ;(n-1) m}^{\prime}$ with $\alpha \in J_{m-1}\left(d^{\prime}\right)$, where $n \in N$ is larger than $\mathrm{k} / \mathrm{m}$.

Similar statements are also valud relative to the $L_{\hat{p}}$-spaces.

Proof. Let $n \in \boldsymbol{N}$. For large $\lambda>0$ define $r_{\lambda, n}: L_{p} \rightarrow L_{p}$ by

$$
\left(r_{\lambda, n} \varphi\right)(g)=\int_{G} d \hat{h} R_{\lambda}^{(n), g}\left(g h^{-1}\right) \varphi(h) .
$$

If $n=0$ we define $r_{\lambda, 0}=I$. By the bounds of Proposition 3.2.II it follows that $r_{\lambda, n}$ is a continuous operator.

Let $\varphi, \phi \in C_{c}^{\infty}(G)$. It follows as in the proof of (19) that for all large $\lambda>0$ and $n \in \boldsymbol{N}$

$$
\begin{aligned}
& \left(\left(\lambda I+H^{\dagger}\right) \psi, r_{\lambda, n} \varphi\right) \\
& \quad=\left(\psi, r_{\lambda, n-1} \varphi\right)+(n-1) !^{-1} \int_{G} d g \int_{G} d \hat{h} \int_{0}^{\infty} d t e^{-\lambda t} t^{n-1} \overline{\psi(g)} L_{t}(g ; h) \varphi(h) .
\end{aligned}
$$

Now for all $g, h \in G$ fixed, $g+h$, one has

$$
\begin{aligned}
(n-1) !^{-1} \int_{0}^{\infty} d t e^{-\lambda t} t^{n-1} L_{t}(g ; h) & \\
= & (n-1) !^{-1} \int_{0}^{\infty} d t e^{-\lambda t} t^{n-1} \sum c_{\alpha}(g)\left(A^{\beta} \partial^{r} K_{t}^{g}\right)\left(g h^{-1}\right) \\
& =(n-1) !^{-1} \sum c_{a}(g) \partial^{r} \int_{0}^{\infty} d t e^{-\lambda t} t^{n-1}\left(A^{\beta} K_{t}^{g}\right)\left(g h^{-1}\right) \\
& =\sum c_{a}(g)\left(\partial^{r} A^{\beta} R_{\lambda}^{(n), g}\right)\left(g h^{-1}\right)
\end{aligned}
$$

where the sum is over all $\alpha$ with $|\alpha|=m$ and all $(\beta, \gamma) \in L b(\alpha)$ with $|\beta| \neq m$. So

$$
\left(\left(\lambda I+H^{\dagger}\right) \psi, r_{\lambda, n} \varphi\right)=\left(\phi, r_{\lambda, n-1} \varphi\right)+\left(\psi, T_{\lambda}^{n, 1} \psi\right)
$$

where we can define the operator $T_{\lambda}^{n, 1}: L_{p} \rightarrow L_{p}$ by

$$
\left(T_{\lambda}^{n, 1} \varphi\right)(g)=\sum_{\substack{\beta, \gamma \in J m\left(d^{\prime}\right) \\|\beta| \neq m}} c_{1, \beta, \gamma}(g) \int_{G} d \hat{h}\left(\Lambda^{\beta} \partial^{r} R_{\lambda}^{n, g}\right)\left(g h^{-1}\right) \varphi(h)
$$

because of the bounds of Proposition 3.2.II. Here $c_{1, \beta, \gamma}=c_{\alpha}$ if $(\beta, \gamma) \in L b(\alpha)$ and $|\beta| \pm m$, and $c_{1, \beta, \gamma}=0$ otherwise. Note that $T_{\lambda}^{n, 1}$ is continuous. Then by density, Lemma 2.4, it follows that (26) is valid for all $\varphi \in L_{p}$ and $\phi \in L_{p ; m}^{\prime}$. So $r_{\lambda, n} \varphi \in D\left(\left(\lambda I+H^{\dagger}\right)^{*}\right)=D(\lambda I+\bar{H})$ and

$$
\left(\phi,(\lambda I+\bar{H}) r_{\lambda, n} \varphi\right)=\left(\phi, r_{\lambda, n-1} \varphi\right)+\left(\phi, T_{\lambda}^{n, 1} \phi\right)
$$

first for all $\varphi \in L_{p}$ and $\phi \in L_{q ; m}^{\prime}$ and by density for all $\phi \in L_{q}$. Next we prove by induction that for all $k \in\{2, \cdots, n\}$ there exist $c_{k, \beta, r} \in C_{b}(G)$, where $\beta, \gamma \in$ $J_{k m}\left(d^{\prime}\right),|\beta| \neq k m$, which are polynomials in the $A^{\delta} c_{\alpha}$ with $\delta \in J_{(k-1) m}\left(d^{\prime}\right)$ and 
$\alpha \in J_{m}\left(d^{\prime}\right)$, such that if $T_{\lambda}^{n, k}$ is the continuous operator on $L_{p}$ defined by

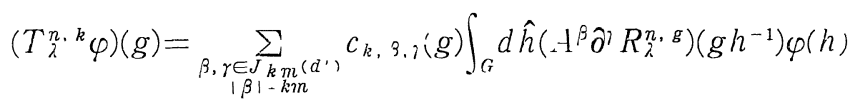

then

$$
\left(H^{\dagger} \phi, T_{\lambda}^{n, k-1} \varphi\right)=\left(\phi, T_{\lambda}^{n, k} \varphi\right)
$$

for all $\phi \in L_{q ; m}^{\prime}$ and $\varphi \in L_{p}$. Now if $\varphi, \phi \in C_{c}^{\infty}(G)$ then

$$
\begin{aligned}
& \left(H^{\dagger} \phi, T_{\lambda}^{n, k-1} \varphi\right) \\
& =\sum_{\substack{\beta, \gamma \in J \in J-1) m\left(a^{\prime}\right) \\
|\beta| \neq(k-1) m}} \int_{G} d \hat{h} \int_{G} d g \overline{\left(H^{\dagger} \phi\right) \overline{(g)}} c_{k-1, \beta, \gamma}(g)\left(A^{\beta} \partial^{r} R_{\lambda}^{n, g}\right)\left(g h^{-1}\right) \varphi(h) .
\end{aligned}
$$

For fixed $h \in G$ the function

$$
\Phi(g)=c_{k-1,3, \lambda}(g)\left(H^{\beta} \partial^{r} R_{\lambda}^{n, g}\right)\left(g h^{-1}\right)
$$

from $G \backslash\{h\}$ into $C$ satisfies the conditions of Lemma 3.3 because of the first bounds in Lemma 3.1.I, equation (25) and Proposition 3.2.I and it follows by induction that the function $\Phi$ is $m$-times differentiable in the $L_{1}$-sense and $\left(H^{\dagger} \psi, \Phi\right)=(\phi, H \Phi)$. From this the existence of the functions $c_{k, \beta, \gamma}$ including the prescribed properties follows from the Leibniz formula. Then (28) is valid for all $\varphi, \phi \in C_{c}^{\infty}(G)$ and by density for all $\varphi \in L_{p}$ and $\phi \in L_{q ; m}^{\prime}$. Note that

$$
\left\|T_{\lambda}^{n, k}\right\|_{p \rightarrow p} \leqq c_{n, k} \lambda^{-(n m-k m+1) / m}
$$

for all large $\lambda$, where $c_{n, k}>0$ depends continuously on $\mu,\|C\|_{k m}^{\prime}$ and the $\left\|c_{\alpha}\right\|_{\infty ;(k-1) m}^{\prime}$, where $\alpha \in J_{m-1}\left(d^{\prime}\right)$.

Next we shall prove that for all $k \in\{1, \cdots, n\}$ one has $r_{\lambda, n} \varphi \in D\left((\lambda I+\bar{H})^{k}\right)$ and

$$
\left(\phi,(\lambda I+\bar{H})^{k} r_{\lambda, n} \varphi\right)=\left(\phi, r_{\lambda, n-k} \varphi\right)+\sum_{l=1}^{k} \sum_{j=1}^{l}\left(\begin{array}{l}
l \\
j
\end{array}\right) \lambda^{l-j}\left(\phi, T_{\lambda}^{n-k+l, j} \varphi\right)
$$

for all $\varphi \in L_{p}$ and $\phi \in L_{q}$. The case $k=1$ is just (27). Now suppose that (29) is valid for some $k \in\{1, \cdots, n-1\}$. Then for all $\varphi \cong L_{p}$ and $\phi \in L_{q ; m}^{\prime}$ one has

$$
\begin{aligned}
((\lambda I+ & \left.\left.H^{\dagger}\right) \phi,(\lambda I+\bar{H})^{k} r_{\lambda, n} \varphi\right) \\
= & \left(\left(\lambda I+H^{\dagger}\right) \psi, r_{\lambda, n-k} \varphi\right)+\sum_{l=1}^{k} \sum_{j=1}^{l}\left(\begin{array}{c}
l \\
j
\end{array}\right) \lambda^{l-j}\left(\left(\lambda I+H^{\dagger}\right) \psi, T_{\lambda}^{n-k+l, \jmath} \varphi\right) \\
= & \left(\phi, r_{\lambda, n-k-1} \varphi\right)+\left(\psi, T_{\lambda}^{n-k, 1} \varphi\right) \\
& +\sum_{l=1}^{k} \sum_{j=1}^{l}\left(\begin{array}{l}
l \\
j
\end{array}\right)\left(\lambda^{l-\jmath+1}\left(\psi, T_{\lambda}^{n-k+l, \jmath} \varphi\right)+\lambda^{l-j}\left(\psi, T_{\lambda}^{n-k+l, j+1} \varphi\right)\right) .
\end{aligned}
$$

So $(\lambda I+\bar{H})^{k} r_{\lambda, n} \varphi \in D\left(\left(\lambda I+H^{\dagger}\right)^{*}\right)=D(\lambda I+\bar{H})$ and (29) is valid for all $\varphi \in L_{p}$ and $\phi \Subset L_{q}^{\prime}$, and then, by density, for all $\phi \in L_{q}$.

By induction it follows that $r_{\lambda, n} \varphi \in D\left((\lambda I+\bar{H})^{n}\right)$ for all $\varphi \in L_{p}$ and 
where

$$
(\lambda I+\bar{H})^{n} r_{\lambda, n}=I+Q_{\lambda}^{(n)}
$$

$$
Q_{\Lambda}^{(n)}=\sum_{l=1}^{n} \sum_{j=1}^{l}\left(\begin{array}{l}
l \\
j
\end{array}\right) \lambda^{l-j} T_{\lambda}^{l, j}
$$

Now

$$
\left\|Q_{\lambda}^{(n)}\right\|_{p \rightarrow p} \leqq \sum_{l=1}^{n} \sum_{j=1}^{l}\left(\begin{array}{c}
l \\
j
\end{array}\right) \lambda^{l-j}\left\|T_{\lambda}^{l, j}\right\|_{p \rightarrow p} \leqq c \sum_{l=1}^{n} \sum_{j=1}^{l}\left(\begin{array}{l}
l \\
j
\end{array}\right) \lambda^{l-j} \lambda^{-(l m-j m+1) / m}=c^{\prime} \lambda^{-1 / m} .
$$

So $\left\|Q_{\lambda}^{(n)}\right\|_{p \rightarrow p} \leqq 2^{-1}$ for large $\lambda$ and the operator $I+Q_{\lambda}^{(n)}$ is invertible. Moreover.

$$
(\lambda I+\bar{H})^{-n}=r_{\lambda, n}\left(I+Q_{\lambda}^{(n)}\right)^{-1}
$$

if $\lambda$ is large enough. Now it follows as for the operators $T^{n, k}$ that $r_{\lambda, n}$ maps $L_{p}$ continuously into $L_{p ; n m-1}^{\prime}$ and $\left\|A^{\alpha} r_{\lambda, n}\right\|_{p \rightarrow p} \leqq c \lambda^{-(n m-|\alpha|) / m}$ for large $\lambda$ and all $\alpha \in J_{n m-1}\left(d^{\prime}\right)$, where $c$ depends only on $\mu,\|C\|_{n m}^{\prime}$ and the $\left\|c_{\alpha}\right\|_{\infty ;(n-1) m}^{\prime}$ with $\alpha \sqsubseteq J_{m-1}\left(d^{\prime}\right)$. So $(\lambda I+\bar{H})^{-n}$ maps $L_{p}$ continuously into $L_{p ; n m-1}^{\prime}$ and $\| A^{a}(\lambda I+$ $\bar{H})^{-n} \|_{p \rightarrow p} \leqq 2 c \lambda^{-(n m-|\alpha|) / m}$ for all large $\lambda>0$. Now the $L_{p}$-statements of the theorem follow as in the end of the proof of Theorem 5.3 in [10]. The proof of the $L_{\hat{p}}$-statements is very similar.

Corollary 3.5. For all $t>0$ one has $K_{t} \in C_{b ; \infty}(G \times G)$.

Proof. Let $L$ be the left regular representation of $G \times G$ in $\mathcal{L}_{\infty}=L_{\infty}(G \times$ $G ; d g \times d g)$. The Lie algebra of $G \times G$ can be identified with $g \oplus g$ and $\left(a_{i}, 0\right)$, $\left(0, a_{i}\right)$, with $i \subseteq\left\{1, \cdots, d^{\prime}\right\}$ form an algebraic basis for $g \oplus g$. We denote the corresponding infinitesimal generators by $A_{1, \imath}$ and $A_{2, \imath}$, respectively. Let $T$ be the holomorphic semigroup on $\mathcal{L}_{1}=L_{1}(G \times G ; d g \times d g)$ used in the proof of Step 5 of the proof of Theorem 2.1. Let $H_{1}$ be the generator of $T$. Then $\varphi \otimes \phi \in$ $D\left(H_{1}\right)$ for all $\varphi \in L_{1 ; \infty}$ and $\phi \boxminus L_{1}$, and $H_{1}(\varphi \otimes \psi)=\left(H^{\dagger} \varphi\right) \otimes \psi$. There exists $\lambda>0$ such that $\lambda I+\overline{H^{1}}$ and $\lambda I+H_{1}$ are invertible. Let $n \in \boldsymbol{N}$. Then $\left(\lambda I+H_{1}\right)^{-n}$ is an extension of the restriction of $\left(\lambda I+\overline{H^{\dagger}}\right)^{-n} \otimes I$ to $L_{1 ; \infty} \otimes L_{1}$. Now the map $\varphi \mapsto$ $\left(\lambda I+\overline{H^{\dagger}}\right)^{-n} A_{i}^{n} \varphi$ from $L_{1 ; \infty} \subset L_{1}$ into $L_{1}$ is bounded by Theorem 3.4.II. Let $B_{n}$ be its unique continuous extension on $L_{1}$, which exists since $L_{1 ; \infty}$ is norm dense in $L_{1}$. The map $B_{n} \otimes I$ from $L_{1} \otimes L_{1}$ into $\mathcal{L}_{1}$ extends to a unique continuous map $\overline{B_{n} \otimes I}$ from $\mathcal{L}_{1}$ into $\mathcal{L}_{1}$. Now let $\varphi_{1} \in L_{1 ; \infty}, \varphi_{2} \in L_{\infty ; \infty}, \phi_{1} \in L_{1}$ and $\phi_{2} \in L_{\infty}$. Then

$$
\begin{aligned}
\left(A_{1,2}^{n}\left(\varphi_{1} \otimes \psi_{1}\right),\left(\lambda I+H_{1}^{*}\right)^{-n}\left(\varphi_{2} \otimes \psi_{2}\right)\right) & =\left(\left(\lambda I+H_{1}\right)^{-n} A_{1,2}^{n}\left(\varphi_{1} \otimes \psi_{1}\right), \varphi_{2} \otimes \psi_{2}\right) \\
& =\left(\left(\left(\lambda I+\overline{H^{\dagger}}\right)^{-n} A_{\imath}^{n} \varphi_{1}\right) \otimes \psi_{1}, \varphi_{2} \otimes \psi_{2}\right) \\
& =\left(\left(B_{n} \otimes I\right)\left(\varphi_{1} \otimes \psi_{1}\right), \varphi_{2} \otimes \psi_{2}\right) \\
& \left.=\left(\varphi_{1} \otimes \psi_{1}, \overline{B_{n} \otimes I}\right)^{*}\left(\varphi_{2} \otimes \psi_{2}\right)\right) .
\end{aligned}
$$

So

$$
\left(A_{1,2}^{n} \xi_{1},\left(\lambda I+H_{1}^{*}\right)^{-n} \xi_{2}\right)=\left(\xi_{1},\left(\overline{B_{n} \otimes I}\right)^{*} \xi_{2}\right)
$$


for all $\xi_{1} \in L_{1 ; \infty} \otimes L_{1}$ and $\xi_{2} \in L_{\infty ; \infty} \otimes L_{\infty}$. But $L_{1 ; \infty} \otimes L_{1}$ is dense in $D\left(A_{1, i}^{n}\right)$, in the $L_{1}$-sense, and $L_{\infty ; \infty} \otimes L_{\infty}$ is weakly* dense in $\mathcal{L}_{\infty}$. Therefore (30) is valid for all $\xi_{1} \in D\left(A_{1,2}^{n}\right)$ and $\xi_{2} \in \mathcal{L}_{\infty}$. But then $\left(\lambda I+H_{1}^{*}\right)^{-n} \xi_{2} \in D\left(A_{1, i}^{n}\right)$, in the $L_{\infty}$-sense, for all $\xi_{2} \in \mathcal{L}_{\infty}$ and $(-1)^{n} A_{1, \iota}^{n}\left(\lambda I+H_{1}^{*}\right)^{-n}=\left(\overline{B_{n} \otimes I}\right)^{*}$. But this implies that $D\left(\left(H_{1}^{*}\right)^{n}\right) \subseteq D\left(A_{1,2}^{n}\right)$, in the $L_{\infty}$-sense. In particular, for all $t>0$ and all $n \in \boldsymbol{N}$ we have

$$
K_{t}=T_{t / 2}^{*} K_{t / 2} \in D\left(\left(H_{1}^{*}\right)^{n}\right) \subseteq D\left(A_{1, \imath}^{n}\right)
$$

in the $L_{\infty}$-sense.

Next we argue that $K_{t} \in D\left(A_{2,2}^{n}\right)$ for all $t>0, n \in N$ and $i \in\left\{1, \cdots, d^{\prime}\right\}$. If we have shown this, then

$$
K_{t} \in \bigcap_{i=1}^{d^{\prime}} D^{\infty}\left(A_{1,2}\right) \cap D^{\infty}\left(A_{2,2}\right)=L_{\infty ; \infty}(G \times G ; d g \times d g)
$$

by [10] Corollary 6.2 and we have proved the corollary.

It remains to prove that $K_{t} \in D\left(A_{2,2}^{n}\right)$. For every $m$-th order form $C$ let the form $C^{c}$ be defined by $C^{c}(\alpha, g)=\overline{C(\alpha, g)}$ for all $\alpha \in J_{m}\left(d^{\prime}\right)$ and $g \in G$. Then $C^{c}(\alpha, g)=(-1)^{|\alpha|} C^{\dagger}\left(\alpha_{*}, g\right)$ and since $a_{d^{\prime}}, \cdots, a_{1}$ is an algebraic basis with the same step as $a_{1}, \cdots, a_{d^{\prime}}$, it follows that $C^{c}$ is subcoercive of step $r$ if and only if $C$ is subcoercive of step $r$. Then if $H^{c}, S^{c}$ and $K^{c}$ are the corresponding operator, semigroup and kernel it follows that $H^{c} \varphi=\overline{H \bar{\varphi}}, S_{t}^{c} \varphi=\overline{S_{t} \bar{\varphi}}$ and $K_{t}^{c}=\bar{K}_{t}$. We need one more transformation of subcoercive forms. For $i \in\left\{1, \cdots, d^{\prime}\right\}$ one has $\Delta A_{2} \Delta^{-1}=A_{2}-\delta_{2} I$, with $\delta_{2}=\left(A_{2} \Delta\right)(e)$. We use the multi-index notation $\delta^{n}$. For every $m$-th order form $C$ let $C^{\Delta}$ be the $m$-th order form defined by

$$
C^{\Delta}(\gamma, g)=\sum_{a \in J J^{\left(d^{\prime}\right)}} \sum_{\substack{\beta \in J m\left(d^{\prime}\right) \\(\beta, \gamma) \in L b(a)}}(-1)^{|\beta|} c_{\alpha}(g) \delta^{\beta}
$$

where $\gamma \in J_{m}\left(d^{\prime}\right)$ and $g \in G$. The principal part of $C^{\Delta}$ equals the principal part of $C$, so $C^{\Delta}$ is subcoercive if, and only if, $C$ is subcoercive. If $C^{\Delta}$ is subcoercive and $H^{\Delta}, S^{\Delta}$ and $\kappa^{\Delta}$ are the corresponding operator, semigroup and kernel, then $H^{\Delta} \varphi=\Delta H \Delta^{-1} \varphi$ for all $\varphi \in C_{c}^{\infty}(G)$. For $t>0$ define $M_{t}: G \times G \rightarrow C$ by

$$
M_{t}(g ; h)=\Delta(g) \Delta(h)^{-1} K_{t}(g ; h) .
$$

Then $M_{t}$ satisfies the same kind of Gaussian bounds as $K_{t}$ since $\Delta\left(g h^{-1}\right) \leqq$ $a e^{\rho\left|g h^{-1}\right|^{\prime}}$ for some $a, \rho>0$. Now let $T_{t}$ be the map from $L_{2}$ into $L_{2}$ as in the proof of Step 3 of the proof of Theorem 2.1, but with $M_{t}$ instead of $K_{t}$. So

$$
\left(T_{t} \varphi\right)(g)=\int_{G} d \hat{h} M_{t}(g ; h) \varphi(h) .
$$

Moreover, let

$$
R_{\lambda}(g ; h)=\int_{0}^{\infty} d t e^{-\lambda t} M_{t}(g ; h)
$$

and define the operator 


$$
\left(r_{\lambda} \varphi\right)(g)=\int_{G} d \hat{h} R_{\lambda}(g ; h) \varphi(h),
$$

for large $\lambda>0$ and $\varphi \in L_{2}$. By a calculation similar to that used to deduce (21) one proves that

$$
\left(\left(\lambda I+\left(H^{\Delta}\right)^{\dagger}\right) \phi, r_{\lambda} \varphi\right)=(\phi, \varphi)
$$

for all $\varphi, \phi \in C_{c}^{\infty}(G)$ and large $\lambda>0$. Now it follows as in the proof of Step 3 of Theorem 2.1 that $S^{\lrcorner}=T_{t}$ and hence $K_{t}^{\Delta}=M_{t}$. So

$$
K_{t}^{\Delta}(g ; h)=\Delta(g) \Delta(h)^{-1} K_{t}(g ; h) .
$$

Now we finish the proof of the corollary. Since $K$ is the kernel associated with the subcoercive form $C$ it follows from Theorem 2.1.IV and the above that the kernel $K^{+c \Delta}$ corresponding to the form $C^{r c \Delta}$ satisfies

$$
K_{t}(g ; h)=K_{t}^{+c \Delta}(h ; g)
$$

for all $g, h \in G$. As above, $K_{t}^{\dagger c \Delta} \in D\left(A_{1,2}^{n}\right)$ for all $n$, so $K_{t} \in D\left(A_{2,2}^{n}\right)$ for all $n \in N$ and this completes the proof.

Corollary 3.6. For all $\alpha \in J\left(d^{\prime}\right), t>0$ and $p \in[1, \infty]$ one has $A^{n} K_{t} \in \mathcal{L}_{p}^{0}$ and

$$
\left(A^{\alpha} S_{t} \varphi\right)(g)=\int_{G} d \hat{h} A^{\alpha} K_{t}(g ; h) \varphi(h)
$$

for all $\varphi \in L_{\hat{q}}$ and $g \in G$, where $q$ is dual to $p$. Moreover, there exists $c>0$ such that

$$
\left\|A^{\alpha} K_{t}\right\|_{p}^{0} \leqq c t^{-D^{\prime} /(m q)} t^{-|\kappa| / m}
$$

for all $p \in[1, \infty]$ and $t>0$.

Proof. Let $t>0$. Then for all $\varphi \in C_{c}^{\infty}(G)$ we have

$$
\left(S_{t} \varphi\right)(g)=\int_{G} d \hat{h} K_{t}(g ; h) \varphi(h)
$$

for all $g \in G$. Moreover, $S_{t} \varphi$ and $K_{t}$ are $C^{\infty}$-functions. So

$$
\left(A^{\varkappa} S_{t} \varphi\right)(g)=\int_{G} d \hat{h} A^{\wedge} K_{t}(g ; h) \varphi(h)
$$

for all $g \in G$. Then one obtains

$$
\begin{aligned}
\left\|A^{\alpha} K_{t}\right\|_{p}^{0} & =\sup _{g \in G}\left(\int_{G} d \hat{h}\left|\left(A^{\alpha} K_{t}\right)(g ; h)\right|^{p}\right)^{1 / p} \\
& =\sup _{g \in G} \sup _{\varphi \in C_{c}^{\infty}(G)}\left|\int_{G} d \hat{h} A^{\alpha} K_{t}(g ; h) \varphi(h)\right| \\
& =\sup _{g \in G} \sup _{\substack{\varphi \in C^{\infty}(G) \\
\|(|\varphi| \hat{q} \leq 1}}\left|\left(A^{\alpha} S_{t} \varphi\right)(g)\right| \\
& \leqq\left\|A^{\alpha} S_{t}\right\|_{\hat{q} \rightarrow \infty} \leqq\left\|A^{\alpha} S_{t / 2}\right\|_{\infty \rightarrow \infty}\left\|S_{t / 2}\right\| \hat{q} \rightarrow \infty \leqq c_{1}(t / 2)^{-1 a \mid / m} c_{2}(t / 2)^{-D^{\prime} /(m q)}
\end{aligned}
$$


But then it follows that the left and right hand side of (31) are continuous functions of $\varphi$ in $L_{\hat{p}}$ and by density (31) is valid for all $\varphi \in L_{\hat{p}}$.

Our next aim is to prove regularity on $L_{2}$ and $L_{\hat{2}}$.

Theorem 3.7. Suppose the semigroup $S$ generated by the closure $\bar{H}$ of $H=$ $d L(C)$ on $L_{2}$ is exponentially decreasing. Then one has the following.

I. The operator $d L(C)$ is closed.

II. If $n \in \boldsymbol{N}$ then $L_{2 ; n}^{\prime}=D\left(H^{n / m}\right)$ and the norms $\|\cdot\|_{2 ; n}^{\prime}$ and $\varphi \mapsto\left\|H^{n / m} \varphi\right\|_{2}$ are equivalent.

III. There exist $p, q>0$ such that

$$
\operatorname{Re}(H \varphi, \varphi) \geqq p\left(\|\varphi\|_{2 ; m / 2}^{\prime}\right)^{2}-q\|\varphi\|_{2}^{2}
$$

for all $\varphi \in L_{2 ; \infty}$.

IV. For all $\theta \in\left\langle 0, \theta_{C}\right\rangle$ there exists $\left.\omega\right\rangle 0$ such that $\left\|S_{z}\right\|_{2 \rightarrow 2} \leqq e^{\omega|z|}$ for all $z \in$ C with $|\arg z| \leqq \theta$.

Analogous statements are valid relative to left translations on $L_{\hat{2}}$.

Proof. The proof is a minor modification of the corresponding proof for subcoercive operators with constant coefficients, Theorem 6.3 of [10]. Only one part of the argument requires more care. In the proof of Theorem 6.3.I in [10] the Helffer-Nourrigat theorem [15], Theorem 2.1, is used to prove that $C^{1} C$ is a subcoercive form whenever $C$ is a subcoercive form with constant coefficients. The Helffer-Nourrigat theorem implies that there exists a constant $c>0$ such that

$$
\left\|\partial L_{G}(P) \varphi\right\|_{2} \geqq c N_{2 ; m}^{\prime}(\varphi)
$$

for all purely $m$-th order subcoercive forms $P$ with constant coefficients and all $\varphi \in L_{2 ; m}^{\prime}(\tilde{G} ; d \tilde{g})$. But the constant $c$ depends on $P$ and it is not immediately clear that $c$ depends continuously on the $c_{a}$ and the ellipticity constant of $P$. We need this kind of more uniform constant.

The purely $m$-th order forms $P$ (with constant coefficients) form a finite dimensional vector space $V$, isomorphic with $\boldsymbol{R}^{\left(d^{\prime}\right) m}$. Let $\Phi: \boldsymbol{R}^{\left(d^{\prime}\right)^{11}} \rightarrow V$ be an isomorphism. Let $M, \mu>0$ and introduce $W$ by

$$
W=\left\{P \in V:\left\|\Phi^{-1}(P)\right\|_{2} \leqq M \text { and the ellipticity constant of } P \text { is } \geqq \mu\right\} .
$$

Then $W$ is a compact set. We claim that there exists $c>0$ such that for all $P \in W$ and all $\varphi \in L_{2 ; m}^{\prime}(\tilde{G} ; d \tilde{g})$ we have

$$
\left\|\partial L_{\grave{G}}(P) \varphi\right\|_{2} \geqq c N_{2 ; m}^{\prime}(\varphi) .
$$

Suppose this is not the case. Then for all $n \in \boldsymbol{N}$ there exist $P_{n} \in W$ and $\varphi_{n} \in$ $L_{2 ; m}^{\prime}(\tilde{G} ; d \tilde{g})$ such that 


$$
\left\|\partial L_{G}\left(P_{n}\right) \varphi_{n}\right\|_{2}<n^{-1} N_{2 ; m}^{\prime}\left(\varphi_{n}\right) .
$$

Since $W$ is compact, the sequence $P_{1}, P_{2}, \cdots$ has a convergent subsequence. We may assume that $P_{1}, P_{2}, \cdots$ is already convergent. Let $P$ be its limit. By the Helffer-Nourrigat theorem there exists $c>0$ such that

$$
\left\|\partial L_{\tilde{G}}(P) \varphi\right\|_{2} \geqq c N_{2 ; m}^{\prime}(\varphi)
$$

for all $\varphi \in L_{2 ; m}^{\prime}(\tilde{G} ; d \tilde{g})$. There exists $N \in N$ such that $\left\|\hat{o} L_{G}\left(P-P_{n}\right) \varphi\right\|_{2} \leqq$ $2^{-1} c N_{2 ; m}^{\prime}(\varphi)$ for all $\varphi \in L_{2 ; m}^{\prime}(\tilde{G})$. Then for all $n \geqq N$

$$
c N_{2 ; m}^{\prime}\left(\varphi_{n}\right) \leqq\left\|\partial L_{G}(P) \varphi_{n}\right\|_{2} \leqq\left\|\partial L_{G}\left(P_{n}\right) \varphi_{n}\right\|_{2}+2^{-1} c_{2} V_{2 ; m}^{\prime}\left(\varphi_{n}\right),
$$

so

$$
2^{-1} c N_{2 ; n}^{\prime}\left(\varphi_{n}\right) \leqq\left\|\partial L_{G}\left(P_{n}\right) \varphi_{n}\right\|_{2} .
$$

This contradicts (32) for large $n$.

It follows from this that if $C$ is a subcoercive form with variable $C^{\infty}$ coefficients then $\left(C^{\dagger} C\right)(\alpha, g)=\left(C_{g}^{\dagger} C_{g}\right)(\alpha)$ is also a subcoercive form and the $L_{2^{-}}$ statements of the theorem follow.

The $L_{\hat{2}}$-results can be deduced from the $L_{2}$-results by using the observation that if $\varphi \in L_{\hat{2}}$ then $\Delta^{-1 / 2} \varphi \in L_{2}$ and $\|\varphi\|_{\hat{2}}=\left\|\Delta^{-1 / 2} \varphi\right\|_{2}$. For example, if $\varphi \in C_{c}^{\infty}$ one then has

$$
\operatorname{Re}\langle H \varphi, \varphi\rangle=\left(H^{\Delta} \Delta^{-1 / 2} \varphi, \Delta^{-1 / 2} \varphi\right)
$$

where $H^{\Delta}=\Delta^{1 / 2} H \Delta^{-1 / 2}$, acting on $C_{c}^{\infty}$, is the subcoercive operator obtained from $H$ by the replacement $A_{2} \rightarrow A_{2}-2^{-1} \delta_{2} I$ with $\delta_{1}=\left(A_{2} \Delta\right)(e)$. Therefore applying Statement III of the theorem to $H^{\Delta}$ acting on $L_{2}$ one has

$$
\begin{aligned}
\operatorname{Re}\langle H \varphi, \varphi\rangle & \geqq p\left(\left\|\Delta^{-1 / 2} \varphi\right\|_{2 ; m / 2}^{\prime}\right)^{2}-q\left\|\Delta^{-1 / 2} \varphi\right\|_{2}^{2} \\
& \geqq p^{\prime}\left(\|\varphi\|_{\hat{2} ; m / 2}^{\prime}\right)^{2}-q^{\prime}\|\varphi\|_{\hat{2}}^{2}
\end{aligned}
$$

for suitable $p^{\prime}, q^{\prime}>0$. Similarly the other statements follows.

\section{§4. Not-so-smooth Coefficients}

Throughout this section $C$ denotes an $m$-th order subcoercive form with principal coefficients in $L_{\infty}^{\prime} ; m$ and all other coefficients in $L_{\infty}$ and we set $H=$ $d L(C)$. It is convenient to write the operator in the form

$$
H=H_{0}+H_{1}
$$

where

$$
H_{0}=\sum_{\substack{\beta, \gamma \in J\left(d^{\prime}\right) \\|\beta|=|\gamma|=m / 2}} A^{\beta *} c_{\beta, \gamma-H^{\prime}}
$$

with $c_{\beta, \gamma} \in L_{\infty ; m}^{\prime}$ and $H_{1}$ is a differential operator of order $m-1$ with coefficients in $L_{\infty}$. Explicitly one factors each product $A^{a}$ with $|\alpha|=m$ into a product $A^{a}=A^{\beta *} A^{\gamma}$ and sets $c_{\beta, \gamma}=c_{\alpha}$. Then $H_{0}$ differs from the principal part of $H$ by 
an operator of order $m-1$. Next we consider a sequence of regularizations $H_{0}^{(n)}$ of $H_{0}$.

Let $\tau_{n} \in C_{c}^{\infty}$ be the bounded approximation to the identity used in the proof of Lemma 2.4 and define $C^{(n)}$ and $c_{\beta, \gamma}^{(n)}$ by left regularizations of $c_{\beta, \gamma}$ with $\tau_{n}$, i. e.,

$$
C^{(n)}\left(\left\langle\beta_{*}, \gamma\right\rangle, g\right)=c_{\beta, i}^{(n)}(g)=\int_{G} d h \tau_{n}(h) c_{\beta, \gamma}\left(h^{-1} g\right)=\left(L\left(\tau_{n}\right) c_{\beta, \gamma}\right)(g)
$$

where $L$ denotes left translations. Then it follows from the estimates in the proof of Lemma 2.4 that one has bounds

$$
\left\|c_{\beta, \gamma}^{(n)}\right\|_{\infty ; l}^{\prime} \leqq \kappa\left\|c_{\beta, \gamma}\right\|_{\infty ; l}^{\prime}
$$

for some $\kappa>0$ and all $l \in\{0,1, \cdots, m\}$. Moreover, the form $C^{(n)}$ is subcoercive since for all $g \in G$ and $\varphi \in L_{2 ; \infty}(\tilde{G} ; d g)$ one has

$$
\begin{aligned}
\operatorname{Re}\left(\partial L_{\dot{G}}\left(P_{g}^{(n)}\right) \varphi, \varphi\right) & =\int_{G} d h \tau_{n}(h)\left(\operatorname{Re} \sum_{n:|n|=m} c_{\alpha}\left(h^{-1} g\right)\left(A^{a} \varphi, \varphi\right)\right) \\
& =\int_{G} d h \tau_{n}(h) \operatorname{Re}\left(\partial L_{\hat{G}}\left(P_{h^{-1} g}^{(n)}\right) \varphi, \varphi\right) \\
& \geqq \int_{G} d h \tau_{n}(h) \mu^{\prime}\left(N_{2 ; m / 2}^{\prime}(\varphi)\right)^{2} \\
& =\mu\left(N_{2 ; m / 2}^{\prime}(\varphi)\right)^{2},
\end{aligned}
$$

where $P_{g}^{(n)}$ is defined with respect to $C^{(n)}$. So the ellipticity constants $\mu^{(n)}$ of $H_{0}^{(n)}$ are bounded below by the ellipticity constant $\mu$ of $H_{0}$. Similarly

where

$$
\begin{aligned}
\left|\operatorname{Im}\left(\partial L_{\tilde{G}}\left(P_{g}^{(n)}\right) \varphi, \varphi\right)\right| & =\left|\int_{G} d h \tau_{n}(h)\left(\operatorname{Im} \sum_{a:|a|=m} c_{\alpha}\left(h^{-1} g\right)\left({ }^{a} \varphi, \varphi\right)\right)\right| \\
& \leqq \nu \int_{G} d h \tau_{n}(h)\left(N_{2 ; m / 2}^{\prime}(\varphi)\right)^{2} \\
& =\nu\left(N_{2 ; m / 2}^{\prime}(\varphi)\right)^{2}
\end{aligned}
$$

$$
\nu=\sup \left\{\left|\operatorname{Im}\left(\partial L_{G}\left(P_{g}^{(n)}\right) \varphi, \varphi\right)\right| /\left(N_{m / 2}^{\prime}(\varphi)\right)^{2}: g \in G, \varphi \in L_{2}(\tilde{G} ; d g), \varphi \neq 0\right\} .
$$

These latter estimates are useful for the subsequent discussion of holomorphy sectors.

Next introduce the subcoercive operators $H_{0}^{(n)}$ by replacing the coefficients of $H_{0}$ with their regularizations, i. e.,

$$
H_{0}^{(n)}=\sum_{\substack{\beta, \gamma \in J\left(d^{\prime}\right) \\|\beta|=|\gamma|=m / 2}} A^{\beta+} c_{\beta, \gamma}^{(n)} A^{\gamma} .
$$

It follows that the coefficients of $H_{0}^{(n)}$ are in $C_{b ; \infty}$ and the results of Theorem 2.1 are valid for the sequence of operators. Let $S^{(n)}$ denote the interpolating semigroup generated by the closures of $H_{v}^{(n)}$ as a consequence of Theorem 2.1 
and $K^{(n)}$ the corresponding semigroup kernels. Now we consider the convergence of the $S^{(n)}$ and $K^{(n)}$ as $n \rightarrow \infty$ by the methods of [4], Section 11 .

If $S^{(n)}$ acts on $L_{\hat{p}}$ then by Theorem 3.4 one has $S_{t}^{(n)} L_{\hat{p}} \subseteq L_{\hat{p} ; \infty}^{\prime}$ for all $t>0$. Hence one easily obtains by the Duhamel formula

$$
\begin{aligned}
\left\langle\psi,\left(S_{t}^{(n)}-S_{t}^{(l)}\right) \varphi\right\rangle & =-\int_{0}^{t} d s\left\langle\phi, S_{t}^{(n)}\left(H_{0}^{(n)}-H_{0}^{(l)}\right) S_{t-s}^{(l)} \varphi\right\rangle \\
& =-t \int_{0}^{1} d \lambda \sum_{\substack{\beta, \gamma \in \mathcal{J}\left(d^{\prime}\right) \\
|\beta|=\mid i_{i}=m / 2}}\left\langle\tilde{A}^{\beta} \widetilde{S}_{\lambda t}^{(n)} \psi,\left(c_{\beta, \gamma}^{(l)}-c_{\beta, i}^{(l)}\right) \mathcal{H}^{i} S_{(1-\lambda) t}^{(l)} \varphi\right\rangle
\end{aligned}
$$

for all $\varphi \in L_{\hat{p}}$ and all $\phi \in L_{\hat{q}}$ where $q$ is dual to $p$. Here we have used $\tilde{A}$ and $\tilde{S}^{(n)}$ to denote the adjoints of .1 and $S^{(n)}$ with respect to right Haar measure. Thus $\tilde{A}_{l}=-A_{2}+\delta_{l} I$ where $\delta_{1}=\left(A_{2} \Delta\right)(e)$ and $\check{S}^{(n)}$ is the semigroup generated by the closure of the operator $\widetilde{H}_{0}^{(n)}$ obtained from the formal adjoint of $H_{0}^{(n)}$ and the replacement $A_{\imath} \rightarrow \tilde{A}_{\imath}$. Therefore

$$
\left|\left\langle\psi,\left(S_{t}^{(n)}-S_{t}^{(l)}\right) \varphi\right\rangle\right| \leqq t \int_{0}^{1} d \lambda\left\|\tilde{S}_{\Lambda t}^{(n)} \psi\right\|_{\hat{q} ; m / 2}\left\|\left._{1}^{(l)} S_{(1-\lambda) t}^{(l)} \varphi\right|_{\hat{p} ; m / 2}\right\| c^{(n)}-c^{(l)} \|_{\infty}
$$

where

$$
\left\|c^{(n)}-c^{(l)}\right\|_{\infty}=\sum_{|, \beta|=|\gamma|=m / 2}\left\|_{\beta, i} c_{3, i}^{(n)}-c_{\beta, i}^{(l)}\right\|_{\infty} .
$$

But it follows from Statement VII of Theorem 2.1 that one has bounds

$$
\left\|\widetilde{S}_{t}^{(n)} \psi\right\|_{\hat{q} ; m / 2} \leqq a t^{-1 / 2} e^{\omega t}\|\psi\|_{\hat{q}}, \quad\left\|S_{t}^{(l)} \varphi\right\|_{\hat{p} ; m / 2} \leqq a t^{-1 / 2} e^{\omega t}\|\varphi\|_{\hat{p}}
$$

with $a$ and $\omega$ uniform in $l$ and $n$. The values of these parameters can be chosen to depend on the coefficients of $H$ only through the ellipticity constant $\mu$ and $\|C\|_{m}^{\prime}$. These estimates establish that

$$
\left|\left\langle\psi,\left(S_{t}^{(n)}-S_{t}^{(l)}\right) \varphi\right\rangle\right| \leqq a^{2} e^{\omega t} \int_{0}^{1} d \lambda \lambda^{-1 / 2}(1-\lambda)^{-1 / 2}\|\psi\|_{\hat{q}}\|\varphi\| \hat{p}\left\|c^{(n)}-c^{(l)}\right\|_{\infty}
$$

and consequently one deduces that the sequences $S_{t}^{(n)}$ converge uniformly as $n \rightarrow \infty$, since the $c_{\beta, i}$ are left differentiable in the subelliptic directions and hence continuous. Therefore ${ }_{h}^{l} c^{(n)}-c^{(l)} \|_{\infty} \rightarrow 0$ as $n, l \rightarrow \infty$ by the argument given in the proof of Lemma 2.4. The limits automatically form a continuous semigroup $T$. But a similar argument establishes uniform convergence of the $S_{t}^{(n)}$ on the $L_{p}$-spaces and hence the limits $T$ are a consistent interpolating semigroup.

The convergence of the semigroups also has implications for the kernels. Since the $S_{t}^{(n)}$ converge uniformly on $L_{\infty}$ one has

$$
\lim _{l, n \rightarrow \infty} \sup _{g \in G} \int_{G} d \hat{h}\left|K_{t}^{(l)}(g ; h)-K_{t}^{(n)}(g ; h)\right|=0
$$

for all $t>0$. In addition Statement VI of Theorem 2.1 gives Gaussian bounds on $K_{t}^{(n)}$ and its left derivatives with respect to the first variable which are uniform in $n$. But applying similar arguments to the formal adjoint of $H_{0}^{(n)}$ and using the symmetry relation of the kernel given by Statement IV of 
Theorem 2.1 one deduces identical bounds for the left derivatives of the kernel with respect to the second variable. In particular one has bounds

$$
\left|\left(B_{\imath} K_{t}^{(n)}\right)(g ; h)\right| \leqq a t^{-1 / n} e^{\omega t}
$$

for all $t>0$ and $i=1, \cdots, d^{\prime}$ uniform in $g, h$ and $n$. Then, however, one readily deduces from the $\mathcal{L}_{\hat{1}}$-convergence (35) of the kernels and the uniform continuity implied by (36) that

$$
\lim _{l} \sup _{n \rightarrow \infty}\left|K_{g, h \in G}^{(l)}(g ; h)-K_{t}^{(n)}(g ; h)\right|=0
$$

for all $t>0$. Thus the sequences $K_{t}^{(n)}$ are $\mathcal{L}_{\hat{1}^{-}}$, and $\mathcal{L}_{\infty^{-}}$, convergent and hence, by interpolation, $\mathscr{L}_{\hat{p}}$-convergent for all $p \in[1, \infty]$. The limits $K_{t}^{(\infty)}$ automatically correspond to the semigroup kernel of the semigroup $T$ and they inherit Gaussian bounds from the approximants $K_{t}^{(n)}$.

Next we argue that $K_{t}^{(\infty)}$ is $(m-1)$-times differentiable in the first variable and that the derivatives satisfy Gaussian bounds. Define the function $k$ on $G$ by setting $k(g)=K_{\iota}(g ; i)$ and similarly define $k^{(n)}$ by setting $k^{(n)}(g)=K_{\imath}^{(n)}(g ; h)$. Then if $\varphi \in L_{\hat{q} ; n-1}$ and $\alpha \in J_{m-1}\left(d^{\prime}\right)$ it follows from the Gaussian bounds on $A^{a} K_{t}^{(n)}$ that $\varphi \mapsto\left\langle\tilde{A}^{a}{ }^{n} \varphi, k^{(n)}\right\rangle$ is continuous uniformly in $n$. Hence $k$ is $(m-1)$ times differentiable in the subelliptic directions in the $L_{\hat{p}}$-sense for all $p \in[1, \infty]$. Moreover, if $q=1$ one has bounds

$$
\left|\backslash \varphi, t^{n} k\right\rangle\left|\leqq \sup _{n \in N}\right|\left\langle\varphi, t^{n} k^{(n)}\right\rangle i
$$

for all $\varphi \in L_{\hat{1} ; m-1}^{\prime}$ and by continuity for all $p \in L_{\hat{1}}$. Now define $e_{n}$ for $\rho \geqq 0$ by setting $e_{\rho}(g)=\exp \left(\rho\left|g h^{-1}\right|^{\prime}\right)$ then for $\chi \in L_{\mathrm{i}}$ with compact support one has

$$
\left|\left\langle\chi_{e_{o}}, .^{n} k\right\rangle\right| \leqq \sup _{n \in N}\left|\left\langle\chi_{e_{o}}, .^{n} k^{n}{ }^{n)}\right\rangle\right|
$$

and taking the supremum over $\chi$ with $\| \chi_{\|_{\hat{1}}}=1$ gives

$$
e^{\rho\left|g h^{-1}\right|^{\prime}}\left|\left(.^{n} k\right)(g)\right| \leqq \sup _{g \in G} \sup _{n \in N} e^{\rho \mid g h^{-1} l^{\prime}}\left|\left(\left.\right|^{a} k^{(n)}\right)(g)\right| .
$$

But using the Gaussian bounds on $\left(A^{n} k^{(n)}\right)(g)=\left(A^{a} h_{t}^{(n)}\right)(g ; h)$ which are uniform in $n$ one obtains bounds

$$
\left|\left(-A^{a} K_{t}\right)(g ; h)\right| \leqq a e^{-\rho\left|g h^{-1}\right|^{\prime}} t^{-\left(D^{\prime}+|n|\right) / m} e^{(\omega)(i+\rho m) l}
$$

and optimizing over $\rho$ gives the required Gaussian bounds on $\left|\left(4^{n} K_{t}\right)\right|$. An analogous argument gives bounds on the mixed derivatives $\left|\left(A^{a} \partial_{t}^{n} K_{t}\right)\right|$.

The foregoing arguments are the key to the extension of Theorem 2.1 to operators with principal coefficients in $L_{\infty ; m}^{\prime}$.

Theorem 4.1. Let $C$ be an m-th order subcoercive form with coefficients $c_{a} \in$ $L_{\infty}$ if $\alpha \in J_{m-1}\left(d^{\prime}\right)$, but with $c_{a} \in L_{\infty ; m}^{\prime}$ if $|\alpha|=m$. Let $H=d L(C)$.

I. $H$ is closable on each of the $L_{n^{-}}$, and $L_{\hat{p}^{-}}$, spaces and the closures $\bar{H}$ 
generate a consistent interpolating semigroup $S$.

II. $S$ is holomorphic in a sector $\Delta_{\theta}=\{z \in C:|\arg z|<\theta\}$ with $\theta \geqq \theta_{C}$.

III. The action of $S_{t}$ is determined by an integral kernel $K_{t} \in C_{b}(G \times G)$ and $t_{\mapsto} K_{t}(g ; h)$ is analytic from $\langle 0, \infty\rangle$ into $C$ for all $g, h \in G$.

IV. For each $n \in \boldsymbol{N}$ the derivative $\partial_{t}^{n} K_{t}$, with $t>0$ fixed, is $(m-1)$-times $L_{\infty}$ (left-)differentiable in the first variable and there exist $a, b, c>0$ and $\omega \geqq 0$ such that

$$
\left|\left(A^{a} \hat{o}_{t}^{n} K_{t}\right)(g ; h)\right| \leqq a c^{n} n ! t^{-n} t^{-\left(D^{\prime}+|a|\right) / m} e^{\omega t} e^{-b\left(\left(\left|g h^{-1}\right|^{\prime}\right)^{m} t-1\right) 1 /(m-1)}
$$

for all $n \in N, t>0, g, h \in G$ and all multi-indices $\alpha$ with $|\alpha|<m$ where the th are the left derivatives with respect to the first variable. The values of $a, b, c$ and $\omega$ depend on the coefficients $c_{\alpha}$ through the ellipticity constant $\mu$ and the parameter

$$
\|C\|_{m}^{\prime}=\sum_{a:|a|=m}\left\|c_{a}\right\|_{\infty ; m}^{\prime}+\sum_{a \in J} \sum_{m-1\left(d^{\prime}\right)}\left\|c_{\alpha}\right\|_{\infty} .
$$

V. For each $p \in[1, \infty], t>0$ one has $S_{t} L_{p} \leqq L_{p ; m-1}^{\prime}, S_{t} L_{\hat{p}} \subseteq L_{\hat{p}}^{\prime} ; m-1$, there exist $a>0$ and $\omega \geqq 0$, depending on the coefficients $c_{\alpha}$ through $\mu$ and $\|C\|_{m}^{\prime}$, such that

$$
\left\|A^{\alpha} S_{\iota}\right\|_{p \rightarrow p} \leqq a t^{-|\alpha| / m} e^{\omega t}, \quad\left\|A^{a} S_{\iota}\right\|_{\hat{p} \rightarrow \hat{p}} \leqq a t^{-|a| / m} e^{\omega t}
$$

and $A^{a} K_{t}$ is the kernel of $A^{a} S_{t}$, for all $\alpha \in J_{m-1}\left(d^{\prime}\right)$.

Proof. Let $T$ be the semigroup constructed above as the limit of the $S^{(n)}$. The $S^{(n)}$ are all holomorphic and it follows from the construction of the regularizations that their holomorphy sector contains the common sector $\Delta_{\theta_{C}}$. But the proof of uniform convergence of the sequence $n \mapsto S_{t}^{(n)}$ for $t>0$ applies equally well to $S_{z}^{(n)}$ for all $z \in \Delta_{\theta_{C}}$ because the replacement of $t$ by $z=t e^{\imath \theta}$ corresponds to the replacement of the subcoercive operator $H_{0}$ by the operator $e^{i \theta} H_{0}$. Now $\theta_{C}$ is chosen such that $e^{2 \theta} H_{0}$ remains subcoercive for $|\theta|<\theta_{C}$ and the replacement $H_{0} \rightarrow e^{i \theta} H_{0}$ only alters the values of the parameters such as the ellipticity constant which enter the estimates. Therefore $T$ is also holomorphic in a sector containing $\Delta_{\theta_{C}}$.

Next let $H_{0}^{(\infty)}$ denote the generator of $T$ acting on $L_{\hat{p}}$ for some fixed $p \in$ $[1, \infty]$. We wish to argue that $H_{0}^{(\infty)}$ extends $H_{0}$. First for $\varphi \in L_{\hat{p} ; m}^{\prime}$ and $\phi \in$ $L_{\hat{q} ; m / 2}^{\prime}$ one readily checks that

$$
\lim _{n \rightarrow \infty}\left\langle\psi, H_{0}^{(n)} \varphi\right\rangle=\left\langle\psi, H_{0} \varphi\right\rangle .
$$

Therefore for $\varphi \in L_{\hat{p} ; m}^{\prime}$ and $\phi \in L_{\hat{\imath}}$ one has

$$
\left\langle\psi, S_{s}^{(n)}\left(H_{0}^{(n)}-H_{0}\right) \varphi\right\rangle=\lim _{l \rightarrow \infty}\left\langle\psi, S_{s}^{(n)}\left(H_{0}^{(n)}-H_{0}^{(l)}\right) \varphi\right\rangle .
$$

Secondly, using the estimates (34) one obtains bounds

$$
\left|\left\langle\psi, S_{s}^{(n)}\left(H_{0}^{(n)}-H_{0}^{(l)}\right) \varphi\right\rangle\right| \leqq a s^{-1 / 2} e^{\omega s}\|\phi\|_{\hat{q}}\|\varphi\|_{\hat{p} ; m / 2}^{\prime}\left\|c^{(n)}-c^{(l)}\right\|_{\infty}
$$


which are integrable at $s=0$. Hence an elementary calculation together with the Lebesgue dominated convergence theorem gives

$$
\begin{aligned}
& t^{-1}\left\langle\psi,\left(I-T_{t}\right) \varphi\right\rangle-t^{-1} \int_{0}^{t} d s\left\langle\psi, T_{s} H_{0} \varphi\right\rangle \\
& \quad=\lim _{n \rightarrow \infty}\left(t^{-1}\left\langle\psi,\left(I-S_{t}^{(n)}\right) \varphi\right\rangle-t^{-1} \int_{0}^{t} d s\left\langle\psi, S_{s}^{(n)} H_{0}^{(n)} \varphi\right\rangle\right) \\
& \quad=0
\end{aligned}
$$

and it then follows immediately that $H_{0}^{(\infty)}$ extends $H_{0}$.

In order to prove that $H_{0}^{(\infty)}$ is in fact the closure $\bar{H}_{0}$ of $H_{0}$ it now suffices to prove that $R\left(\lambda I+\bar{H}_{0}\right)=L_{\hat{p}}$ for $\operatorname{Re} \lambda$ large. This we will achieve by use of the resolvent estimates of Section 2. First it is convenient to reexpress $H_{0}$ in the original form

$$
H_{0}=\sum_{a \in J m\left(d^{\prime}\right)} c_{a+1}^{\prime}-^{a} .
$$

Then the $c_{a}^{\prime}$ can be identified with the principal coefficients $c_{\beta, \gamma}$ or their left derivatives of order less than or equal to $m / 2$. Similarly the regularizations $H_{0}^{(n)}$ can be expressed in the form

$$
\left.H_{0}^{(n)}=\sum_{n \in J} c_{m} c^{\prime}\right) c_{n, \alpha} \cdot 1^{n}
$$

and the $c_{n}$ a are identifiable with left derivatives, of order less than or equal to $m / 2$, of the regularized coefficients $c_{\beta}^{(n)}$, . Then we let $r_{\Lambda}^{(n)}$ denote the parametrix approximation defined by (18) of the resolvent of the constant operator $H_{0}^{(n), g}$ obtained by fixing the coefficients $c_{n, a}$ with their values at $g$. Now it follows from the estimates of Section 2, together with (33) and the inequalities $\mu^{(n)} \geqq \mu$ for the ellipticity constants, that one has bounds

$$
\left\|r_{\lambda}^{(n)}\right\|_{\hat{p}, \hat{p}} \leqq c(\operatorname{Re} \lambda)^{-1}
$$

if $\operatorname{Re} \lambda$ is sufficiently large, with the value of $c$ independent of $n$ and $\lambda$. Moreover, if $\varphi \in C_{c}^{\infty}$ then $r_{\Lambda}^{(n)} \varphi \in L_{\hat{p} ; m}^{\prime}$ for $\operatorname{Re} \lambda$ large enough (see Step 2 in the proof of Theorem 2.1). Moreover, one has bounds

$$
\left\|r_{\lambda}^{(n)} \varphi\right\|_{\hat{p} ; m} \leqq c(\operatorname{Re} \lambda)^{-1}\|\varphi\|_{\hat{p} ; m r}^{\prime}
$$

with $c$ independent of $n$ and $\lambda$. Therefore one has estimates

$$
\left\|\left(r_{\lambda}^{(n)}-r_{\lambda}^{(l)}\right) \varphi\right\|_{\hat{p}} \leqq c(\operatorname{Re} \lambda)^{-2}\left\|c_{n}-c_{l}\right\|_{\infty}\|\varphi\|_{\hat{p} ; m r}^{\prime}
$$

where we have now defined

Consequently

$$
\left\|c_{n}-c_{l}\right\|_{\infty}=\sum_{a \in J m\left(d^{\prime}\right)}\left\|c_{n, \alpha}-c_{l, \alpha}\right\|_{\infty} .
$$

$$
\lim _{l, n \rightarrow \infty}\left\|\left(r_{\lambda}^{(n)}-r_{\lambda}^{(l)}\right) \varphi\right\|_{\hat{p}}=0
$$


for all $\varphi \in C_{c}^{\infty}$ and $p \in[1, \infty]$. So $r_{\lambda} \varphi=\lim _{n \rightarrow \infty} r_{\lambda}^{(n)} \varphi$ exists for all $\varphi \in C_{c}^{\infty}(G)$. Since $r_{\lambda}^{(n)}$ satisfies the bounds (37), it follows that ${ }_{\|} r_{\lambda} \varphi\left\|_{\hat{p}} \leqq c(\operatorname{Re} \lambda)^{-1}\right\| \varphi \|_{\hat{p}}$ for all $\varphi \in C_{c}^{\infty}(G)$. Hence $r_{\lambda}$ extends to a continuous operator on $L_{\hat{p}}$, still denoted by $r_{\lambda}$, such that $\left\|r_{\lambda}\right\|_{\hat{p} \rightarrow \hat{p}} \leqq c(\operatorname{Re} \lambda)^{-1}$. Next note that the formal adjoints $H_{0}^{\dagger}$ and $H_{0}^{(n)_{\top}}$ of $H_{0}$ and $H_{0}^{(n)}$ are defined on $L_{\hat{q} ; m}^{\prime}$ where $q$ is dual to $p$ and

$$
\lim _{n \rightarrow \infty}\left\|\left(H_{0}^{(n) \top}-H_{0}^{+}\right) \phi\right\|_{\hat{q}}=0
$$

for all $\phi \in L_{\hat{q}}^{\prime} ; n$ by similar estimations. But $H_{0}^{(n) \tau}$ has $C^{\infty}$-coefficients and hence it follows from (19) that

$$
\left\langle\left(\bar{\lambda} I+H_{0}^{(n)+}\right) \psi, r_{\lambda}^{(n)} \varphi\right\rangle=\langle\psi, \varphi\rangle+\left\langle\psi, Q_{\lambda}^{(n)} \varphi\right\rangle
$$

for all $\phi \in L_{\hat{q} ; m}^{\prime}$ and $\varphi \in C_{c}^{\infty}$ where the $Q_{\lambda}^{(n)}$ are bounded operators satisfying bounds

$$
\left\|Q_{\lambda}^{(n)}\right\|_{\hat{p} \rightarrow \hat{p}} \leqq c(\operatorname{Re} \lambda)^{-1 / n}
$$

uniform in $n$ and $\lambda$ for sufficiently large $\operatorname{Re} \lambda$. The uniformity in $n$ again follows from (33) and the bounds $\mu^{(n)} \geqq \mu$. Then the foregoing observations allow one to conclude that

$$
\lim _{n \rightarrow \infty}\left\{\langle\psi, \varphi\rangle+\left\langle\psi, Q_{\lambda}^{(n)} \varphi\right\rangle\right\}=\left\langle\left(\bar{\lambda} I+H_{0}^{\dagger}\right) \psi, r_{\lambda} \varphi\right\rangle
$$

for all $\phi \in L_{\hat{q} ; m}^{\prime}$ and $\varphi \in C_{c}^{\infty}$. This suffices to conclude that the $Q_{\lambda}^{(n)}$ converge to a bounded operator $Q_{i}$ satisfying bounds

$$
\| Q_{\lambda \| \hat{p} \rightarrow \hat{p}} \leqq c(\operatorname{Re} \lambda)^{-1 / m}
$$

for sufficiently large $\operatorname{Re} \lambda$. Since $r_{\Lambda}^{(n)} \varphi \in L_{\hat{p} ; m}^{\prime}$ and $\left\|r_{\lambda}^{(n)} \varphi\right\|_{\hat{p} ; m}^{\prime} \leqq c(\operatorname{Re} \lambda)^{-1}\|\varphi\|_{\hat{j} ; m r}^{\prime}$, uniformly in $n$, it follows that

$$
\left|\left\langle\mathcal{H}^{a} \phi, r_{\lambda} \varphi\right\rangle\right|=\lim _{n \rightarrow \infty}\left|\left\langle H^{a} \phi, r_{\lambda}^{(n)} \varphi\right\rangle\right| \leqq c(\operatorname{Re} \lambda)^{-1}\|\phi\|_{\hat{q}}\|\varphi\|_{\hat{p} ; m r}^{\prime}
$$

for all $\phi \in L_{\hat{\mathbf{q}} ; m}^{\prime}$ and $\varphi \in C_{c}^{\infty}(G)$. So $r_{\lambda} \varphi \in L_{\hat{p} ; m}^{\prime}$ for all $\varphi \in C_{c}^{\infty}(G)$. Therefore

$$
\left(\lambda I+H_{0}\right) r_{\lambda} \varphi=\left(I+Q_{\lambda}\right) \varphi
$$

for $\varphi \in C_{c}^{\infty}$ and then by closure

$$
\left(\lambda I+\bar{H}_{0}\right) r_{\lambda} \varphi=\left(I+Q_{\lambda}\right) \varphi
$$

for all $\varphi \in L_{\hat{\nu}}$. Replacing $\varphi$ by $\left(I+Q_{\lambda}\right)^{-1} \varphi$ one concludes that

$$
\left(\lambda I+\bar{H}_{0}\right) r_{\lambda}\left(I+Q_{\lambda}\right)^{-1} \varphi=\varphi
$$

for all sufficiently large $\operatorname{Re} \lambda$. Thus $R\left(\lambda I+\bar{H}_{0}\right)=L_{\hat{p}}$ and hence $H_{0}^{(\infty)}=\bar{H}_{0}$ on $L_{\hat{p}}$.

The above calculations have been made relative to the $L_{\hat{p}}$-spaces but similar reasoning establishes the same conclusion, $H_{0}^{(\infty)}=\bar{H}_{0}$, on the $L_{p}$-spaces. Therefore at this point we have demonstrated that the $L_{p^{-}}$, and $L_{\hat{p}^{-}}$, closures of $H_{0}$ generate a consistent interpolating semigroup $T$. Next we appeal to per- 
turbation theory.

First note that

$$
\left|\left\langle\tilde{A}^{\alpha \psi} \psi, S_{t}^{(n)} \varphi\right\rangle\right| \leqq a t^{-|a| / m} e^{\omega t}\|\phi\|\left\|_{\hat{q}}\right\| \varphi \|_{\hat{p}}
$$

for all $\alpha \in J_{m-1}\left(d^{\prime}\right)$ and $\phi \Subset L_{\hat{q} ; m}^{\prime}$. Since the bound is uniform in $n$ it follows in the limit $n \rightarrow \infty$ that $T_{t} L_{\hat{p}} \leqq L_{\hat{p}}^{\prime} ; m-1$ and

$$
\left\|A^{a} T_{t}\right\|_{\hat{p} \rightarrow \hat{p}} \leqq a t^{-|a| / m} e^{\omega t},
$$

for all $t>0$. Therefore $T_{t} L_{\hat{p}} \subseteq D\left(H_{1}\right)$ and

$$
\left\|H_{1} T_{t}\right\|_{\hat{p} \rightarrow \hat{p}} \leqq a^{\prime} t^{-(m-1) / m} e^{\omega t}
$$

for all $t>0$ and a suitable $a^{\prime}>0$. Since the right hand side is integrable at $t=0$ it follows that $H_{1}$ is a perturbation of the generator of $T$ in the sense of "time-dependent" perturbation theory (see, for example, [2] Theorem 3.1.33) on each of the $L_{\hat{p}}$-spaces. But an analogous argument demonstrates that $H_{1}$ is also a perturbation on the $L_{p}$-spaces.

The perturbation estimates (38) on the $L_{\hat{p}}$-spaces, and their analogues on the $L_{p}$-spaces, ensure by standard perturbation theory that $\bar{H}=\bar{H}_{0}+H_{1}$ and $\bar{H}$ generates a continuous semigroup $S$ on each of the spaces. The semigroups $S$ and $T$ are related by the series expansion

$$
S_{t}=\sum_{n \geqq 0} S_{t}^{(n)}
$$

with $S_{t}^{(n)}=T_{t}$ and

$$
S_{t}^{(n+1)}=-\int_{0}^{t} d s S_{s}^{(n)} H_{1} S_{t-s}^{(0)} .
$$

The perturbation estimates ensure that the series is uniformly convergent on each of the spaces and hence $S$ is also a consistent interpolating semigroup. Moreover, $S$ is holomorphic with the same holomorphy sector as $T$. In particular the holomorphy sector of $S$ contains $\Delta_{\theta_{C}}$.

The semigroup $T$ has the kernel $K^{(\infty)}$ constructed as the limit of the approximants $K^{(n)}$ and it follows from the estimates on $A^{n} K_{t}$ with $\alpha \in J_{m-1}\left(d^{\prime}\right)$ that

$$
\left|\left(H_{1} K_{t}^{(\infty)}\right)(g ; h)\right| \leqq a t^{-\left(D^{\prime}+m-1\right) / m} e^{\omega t} e^{-b\left(\left(\left.1 g h^{-1}\right|^{\prime}\right)^{m} t^{-1}\right) 1 /(m-1)}
$$

for all $t>0$ and $g, h \in G$ where the left differential operator $H_{1}$ acts on the lirst variable of $K_{t}^{(\infty)}$. Therefore estimating as in the appendix of [4] one deduces that the perturbation series

$$
K_{t}=\sum_{n \geqq 0} \tilde{K}_{t}^{(n)}
$$

defined by setting $\tilde{K}_{t}^{(0)}=K_{t}^{(\infty)}$ and

$$
\tilde{K}_{t}^{(n+1)}=-\left(\tilde{K}^{(n)} \hat{*} H_{1} \tilde{K}^{(0)}\right)_{t}
$$


is uniformly convergent. Moreover, the recursion relation gives

$$
\begin{aligned}
& \sup _{g \in G} \int_{G} d \hat{h} e^{\rho\left|g h^{-1}\right|^{\prime}}\left|\widetilde{K}_{t}^{(n+1)}(g ; h)\right| \\
& \quad \leqq \sup _{g \in G} \int_{G} d \hat{k} e^{\rho\left|g k^{-1}\right|^{\prime}}\left|\tilde{K}_{t}^{(n)}(g ; k)\right| \sup _{k \in G} \int_{G} d \hat{h} e^{\rho\left|k h^{-1}\right|^{\prime}}\left|H_{1} \tilde{K}_{t}^{(0)}(k ; h)\right|
\end{aligned}
$$

for all $\rho \geqq 0$. Therefore using the bounds (39) and solving iteratively one obtains bounds

$$
e^{\rho\left|g h^{-1}\right|^{\prime}}\left|K_{t}(g ; h)\right| \leqq a t^{-D^{\prime} / m} e^{\omega\left(1+\rho^{m}\right) t}
$$

for all $\rho, t>0$ with the values of $a$ and $\omega$ independent of $\rho$ and $t$. Minimizing over $\rho$ then gives the Gaussian bounds on $\left|K_{t}\right|$. Similar arguments establish that $K_{t}$ is $(m-1)$-times differentiable in the first variable, the derivatives are given by the sum of the derivatives of the terms of the perturbation series and the derivatives satisfy appropriate Gaussian bounds. The bounds on the derivatives $\partial_{t}^{n} K_{t}$ and the mixed derivatives are then derived as in the proof of Theorem 2.1.

The proof of the remaining properties of $K$ and $S$ are again a repetition of the arguments of Section 2.

Under slightly more stringent assumptions on the coefficients of the subcoercive operator one can reestablish all the results of Theorem 2.1.

Corollary 4.2. Let $H$ be an m-th order subcoercive operator with coefficients $c_{\alpha} \in L_{\infty ;|\alpha|}^{\prime}$ for all $\alpha \in J_{m}\left(d^{\prime}\right)$. Then all the statements of Theorem 2.1 are valid.

Proof. The assumptions of the corollary are sufficient to ensure that the formal adjoint of $H$ is defined and satisfies the hypotheses of Theorem 4.1. Hence all the conclusions of this theorem are valid for the operator and for its formal adjoint. But then it follows straightforwardly that the semigroup generated by the closure of the formal adjoint has a kernel which is related to the kernel of the semigroup generated by the closure of $H$ by the relation given in Statement IV of Theorem 2.1. Moreover, the extra differentiability give by Statement $\mathrm{V}$ of the theorem follows by use of this adjoint relation as in the proof of Theorem 2.1.

Next we aim to establish improved smoothness properties of $S$ and $K$ by the derivation of more precise regularity properties of $H$. These are defined in terms of Lipschitz spaces which interpolate between the $L_{\infty}^{\prime} ; m$-spaces. In particular if $\left(\mathscr{X}_{1}, \mathscr{X}_{2}\right)$ is an interpolation pair of Banach spaces, $r \in\langle 0,1\rangle$ and $q \in[1, \infty]$ we use $\left(\mathscr{X}_{1}, \mathscr{X}_{2}\right)_{r, q ; K}$ to denote the interpolation space constructed by Peetre's $K$-method (see [5], Definition 3.2.4).

Theorem 4.3. Let $H$ be an $m$-th order subcoercive operator u'th coefficients 
$c_{\alpha} \in L_{\infty ; m}^{\prime}$ for all $\alpha \in J_{m}\left(d^{\prime}\right)$ with $|\alpha|=m$. Let $\gamma \in\langle 0,1\rangle$ and $q \in[1, \infty]$. Then

$$
D(\bar{H}) \cong\left(L_{\infty ; m-1}^{\prime}, L_{\infty ; m}^{\prime}\right)_{r, q ; K}
$$

and there exists $c>0$ such that

$$
\|\varphi\|_{\infty ; m-1 ; \gamma, q ; K} \leqq \varepsilon^{1-\gamma / m}\|\bar{H} \varphi\|_{\infty}+c \varepsilon^{-(m-1+\gamma) / m}\|\varphi\|_{\infty}
$$

for all $\varphi \in D(\bar{H})$ and $\varepsilon \in\langle 0,1]$ where the norm on the left denotes the norm on the interpolation space. Moreover, if $S$ is the corresponding semigroup then

$$
S_{t} L_{p} \subseteq\left(L_{\infty ; m-1}^{\prime}, L_{\infty ; m}^{\prime}\right)_{\gamma, q ; K}, \quad S_{t} L_{\hat{p}} \leqq\left(L_{\infty ; m-1}^{\prime}, L_{\infty ; m}^{\prime}\right)_{\gamma, q ; K}
$$

for all $p \in[1, \infty]$ and $t>0$.

Proof. First we need some general observations on the interpolation spaces. For brevity let

$$
L_{r, q}=\left(L_{\infty}, L_{\infty ; 1}^{\prime}\right)_{T, q ; K}, \quad L_{m-1 ; i, q}=\left(L_{\infty ; m-1}^{\prime}, L_{\infty ; m}^{\prime}\right)_{r, q ; K} .
$$

Now the action of the generators $A_{2}, i=1, \cdots, d^{\prime}$ can be defined by restriction on these spaces and we let $L_{\gamma, q ; n}^{\prime}$ denote the subspace $\bigcap_{\alpha \in J{ }_{n}\left(d^{\prime}\right)} D\left(A^{a}\right)$ on $L_{\gamma, q}$. Then it follows from [9], Theorem 2.1 Statements I and III, that

$$
L_{\gamma, q ; m-1}^{\prime}=L_{m-1 ; \gamma, q}
$$

with equivalent norms. Next let $L_{\gamma ; q}(L)$ denote the $\varphi \in L_{\infty}$ such that

$$
\int_{\Omega} d g\left(|g|^{\prime}\right)^{-D^{\prime}}\left(\left(|g|^{\prime}\right)^{-r}\|(I-L(g)) \varphi\|_{\infty}\right)^{q}<\infty
$$

where $\Omega$ is some bounded open neighbourhood of the identity $e \in G$. Then $L_{\gamma ; q}(L)$ is a Banach space with respect to the norm

$$
\|\varphi\|_{\gamma, q}^{L}=\|\varphi\|_{\infty}+\left(\int_{\Omega} d g\left(|g|^{\prime}\right)^{-D^{\prime}}\left(\left(|g|^{\prime}\right)^{-r}\|(I-L(g)) \varphi\|_{\infty}\right)^{q}\right)^{1 / q} .
$$

These definitions have an obvious modification if $q=\infty$. Moreover, $L_{i ; q}(L)=$ $L_{\gamma ; q}$, with equivalent norms, by [9], Theorem 3.2, for all $\gamma \in\langle 0,1\rangle$ and $q \in$ $[1, \infty]$. Now we aim to prove that if $\varphi \in D(\bar{H})$ then $A^{a} \varphi \in L_{\gamma ; q}(L)$ for all $\alpha \in$ $J_{m-1}\left(d^{\prime}\right)$. Therefore $D(\bar{H}) \subseteq L_{\gamma, q ; m-1}^{\prime}=L_{m-1 ; \gamma, q}$ by the previous identifications.

We begin by assuming that $q=\infty$ and that all coefficients are in $C_{b ; \infty}$ and then prove

$$
\left\|(I-L(k)) A^{a}(\lambda I+\bar{H})^{-1} \varphi\right\|_{\infty} \leqq c\left(|k|^{\prime}\right) r \lambda^{-\left(m-\mid a_{1}-\gamma\right) / m}\|\varphi\|_{\infty}
$$

for all $k \in B_{1}^{\prime}, \alpha \in J_{m-1}\left(d^{\prime}\right), \gamma \in\langle 0,1\rangle$, all large $\lambda>0$ and all $\varphi \in L_{\infty}$, with some constant $c>0$ which depends on the coefficients of $H$ only through $\|C\|_{m}^{\prime}$ and the ellipticity constant $\mu$. Subsequently we use an approximation argument to establish a similar conclusion for the general case with not-so-smooth coefficients. Finally we use the reiteration theorem to remove the condition on $q$. 
Once we have established (40) in general the desired conclusion follows immediately. Moreover, the inequalities of the theorem are also a consequence of these bounds and the equivalence of the norms of the spaces $L_{\gamma, q ; m-1}^{\prime}$ and $L_{m-1 ; \gamma, q}$.

Let $r_{\lambda}$ be the operator which we denoted by $r_{\lambda}^{(0)}$ in (18) and by $r_{\lambda, 1}$ in the proof of Theorem 3.4. Then $(\lambda I+\bar{H})^{-1}=r_{\lambda}\left(I+Q_{\lambda}\right)^{-1}$ for large $\lambda>0$, with $Q_{\lambda}$ a continuous operator with norm less than or equal to $2^{-1}$. So it is enough to prove (40) with the operator $(\lambda I+\bar{H})^{-1}$ replaced by $r_{\lambda}$, i. e., to prove

$$
\left\|(I-L(k)) A^{a} r_{\lambda} \varphi\right\|_{\infty} \leqq c\left(|k|^{\prime}\right)^{r} \lambda^{-(m-|a|-r) / m}\|\varphi\|_{\infty}
$$

under the above restrictions.

Step 1. Suppose $c_{a} \in C_{b ; \infty}$ for all $\alpha \in J_{m}\left(d^{\prime}\right)$ and $q=\infty$.

Let $k \in B_{1}^{\prime}, \alpha \in J_{m-1}\left(d^{\prime}\right)$ and $\varphi \in L_{\infty}$. Further, let $R_{\lambda}^{g}$ be as in Step 2 of the proof of Theorem 2.1. Then

$$
\begin{aligned}
& \left((I-L(k)) 1^{n} r_{\lambda} \varphi\right)(g) \\
& =\sum_{(, \beta, \gamma) \in L b(a)} \int_{G} d h\left(1^{\beta} \partial^{r} R_{\lambda}^{g}\right)(h) \varphi\left(h^{-1} g\right)-\left(1^{\beta} \partial^{r} R_{\lambda}^{k^{-1} g}\right)(h) \varphi\left(h^{-1} k^{-1} g\right) \\
& =\sum_{(\beta, r) \in L b(a)} \int_{G} d h\left(1^{\beta} \partial^{r} R_{\lambda}^{g}\right)(h) \varphi\left(h^{-1} g\right)-\left(1^{3} \partial^{r} R_{\lambda}^{k-1} g\right)\left(k^{-1} h\right) \varphi\left(h^{-1} g\right) \\
& =\sum_{(\beta, \gamma) \in L b(a)} \int_{G} d h\left(\left(A^{\beta} \partial^{r} R_{\lambda}^{g}\right)(h)-\left(A^{\beta} \partial^{r} R_{\lambda}^{g}\right)\left(k^{-1} h\right)\right) \varphi\left(h^{-1} g\right) \\
& \quad+\left(\left(A^{\beta} \partial^{r} R_{\lambda}^{g}\right)\left(k^{-1} h\right)-\left(A^{\beta} \partial^{r} R_{\lambda}^{k^{-1} g}\right)\left(k^{-1} h\right)\right) \varphi\left(h^{-1} g\right)
\end{aligned}
$$

for $g$ almost everywhere in $G$. Therefore if $\|\varphi\|_{\infty} \leqq 1$ then

$$
\begin{aligned}
\left\|(I-L(k)) \cdot A^{a} \gamma_{\lambda} \varphi\right\|_{\infty} \leqq & \sum_{(, 3, \gamma) \in L b(a)} \sup _{g \in G} \int_{G} d h\left|\left((I-L(k)) A^{\beta} \partial^{r} R_{\lambda}^{g}\right)(h)\right| \\
& +\sum_{(, 3, \gamma, \in L b(a)} \sup _{g \in G} \int_{G} d h\left|\left(A^{3} \partial^{\gamma} R_{\lambda}^{g}\right)(h)-\left(\Lambda^{\beta} \partial^{r} R_{\lambda}^{k-1} g\right)(h)\right| .
\end{aligned}
$$

We consider the two terms separately. The second term is the easiest. Let $(\beta, \gamma) \in L b(\alpha)$. Then

$$
\begin{aligned}
& \sup _{g \in G} \int_{G} d h\left|\left(A^{\beta} \partial^{r} R_{\lambda}^{g}\right)(h)-\left(A^{\beta} \partial^{r} R_{\lambda}^{k^{-1} g}\right)(h)\right| \\
& \quad \leqq \int_{G} d h \sup _{g \in G}\left|\left(A^{s} \partial^{r} R_{\lambda}^{g}\right)(h)-\left(A^{\beta} \partial^{r} R_{\lambda}^{k^{-1} g}\right)(h)\right| \\
& \quad \leqq 2 d^{\prime}|k|^{\prime} \max _{i \in\left(1, \cdot d^{\prime}\right)} \int_{G} d h \sup _{g^{\prime} \in G}\left|\left(A^{\beta} \partial_{\imath} \partial^{r} R^{g_{\lambda}^{\prime}}\right)(h)\right| \\
& \quad \leqq 2 c d^{\prime}|k|^{\prime} \lambda^{-(m-1, \beta) / m},
\end{aligned}
$$

where the last inequality follows by the equalities in the proof of Proposition 
3.2 and the independence from $g$ of the bounds in Lemma 2.2.I. The constant $c$ depends continuously on $\mu$ and $\|C\|_{m}^{\prime}$.

The first term is more difficult. Let $(\beta, \gamma) \in L b(\alpha)$. By (25) we can write

$$
\begin{aligned}
\left(\hat{o}^{r} . A^{\beta} R_{\lambda}^{g}\right)(h)= & \sum_{l=2}^{|\eta|+1} \sum_{\substack{a_{2}, \cdots, \alpha_{l} \in J\left(d^{\prime}\right) \\
\left|\alpha_{2}\right|=\left|\alpha_{l}\right|=m}} c_{\alpha_{2}, \cdots, a_{l}}^{l}(g) \int_{0}^{\infty} d t_{1} \cdots \int_{0}^{\infty} d t_{l} e^{-\lambda\left(t_{1}++t_{l}\right)} \\
& \cdot\left(A^{\beta} K_{t_{1}}^{g} * A^{a_{2}} K_{t_{2}}^{g} * \cdots * A^{a_{l}} K_{t_{l}}^{g}\right)(h)
\end{aligned}
$$

where $c_{\alpha_{2}, \cdots, \alpha_{l}}^{l}$ are polynomials in the $A^{j} c_{\varepsilon}$ with $\delta \in J_{|r|}\left(d^{\prime}\right)$ and $\varepsilon \in J\left(d^{\prime}\right)$ with $|\varepsilon|=m$. So we have to estimate

$$
\begin{aligned}
& \sup _{g \in G} \int_{G} d h\left|\int_{0}^{\infty} d t_{1} \cdots \int_{0}^{\infty} d t_{l} e^{-\lambda\left(t_{1}+\cdot+t_{l}\right)}\left((I-L(k)) A^{\beta} K_{l_{1}}^{g} * A^{a_{2}} K_{t_{2}}^{g} * \cdots * A^{\alpha} l K_{t_{l}}^{g}\right)(h)\right| \\
& =\sup _{g \in G} \sup _{\substack{\varphi \in C_{C}^{\infty}(G) \\
\|\varphi\|_{\infty}<1}} \mid \int_{G} d h \int_{0}^{\infty} d t_{1} \cdots \int_{0}^{\infty} d t_{l} e^{-\lambda\left(t_{1}+\cdot+t_{l}\right)} \\
& \cdot\left((I-L(k)) \cdot 1^{\beta} K_{t_{1}}^{g} * .1^{\kappa_{2}} K_{t_{2}}^{g} * \cdots * .4^{\prime \prime l} K_{t_{l}}^{g}\right)(h) \varphi(h) \\
& =\sup _{g \in G} \sup _{h \in G} \sup _{\substack{\varphi \in C_{C}^{\infty}(G) \\
\mid \varphi^{\prime \prime} \infty \leqq 1}} \mid \int_{0}^{\infty} d t_{1} \cdots \int_{0}^{\infty} d t_{l} e^{-\lambda\left(t_{1}+\cdot+t_{l}\right)} \\
& \cdot\left((I-L(k)) A^{\beta} K_{t_{1}}^{g} * \mathcal{H}^{\alpha_{2}} K_{t_{2}}^{g} * \cdots *_{H^{\alpha}}^{\alpha_{l}} K_{t_{l}}^{g} * \check{\varphi}\right)(h) \mid \\
& =\sup _{g \in G} \sup _{\substack{\varphi \in C_{C}^{\infty}(G) \\
\|\varphi\|_{\infty} \leq 1}}\left\|(I-L(k)) A^{\beta}\left(\lambda I+\overline{H_{g}^{P}}\right)^{-1} A^{\alpha_{2}}\left(\lambda I+\overline{H_{g}^{P}}\right)^{-1} \cdots A^{\alpha} l\left(\lambda I+\overline{H_{g}^{P}}\right)^{-1} \check{\varphi}\right\|_{\infty},
\end{aligned}
$$

for all $l \in\{2, \cdots,|\gamma|+1\}$ and all $\alpha_{2}, \cdots, \alpha_{l} \in\left\{1, \cdots, d^{\prime}\right\}^{m}$, where $\check{\varphi}(h)=\varphi\left(h^{-1}\right)$. The last equality is by Lemma 3.1.III. Therefore we have effectively reduced the problem to a comparable problem for operators with constant coefficients. Now for $j \in\{2, \cdots, l\}$ let $\alpha^{\prime}, \alpha^{\prime \prime} \in J_{m-1}\left(d^{\prime}\right)$ be such that $\alpha=\left\langle\alpha^{\prime}, \alpha^{\prime \prime}\right\rangle$ and

$$
\begin{gathered}
\left|\alpha_{l}^{\prime}\right|=l-j+1, \\
\left|\alpha_{l}^{\prime \prime}\right|=m-l+j-1 .
\end{gathered}
$$

Then for all $\varepsilon \in\langle 0,1-\gamma\rangle$ we have

$$
\begin{aligned}
& \sup _{g \in G} \sup _{\substack{\varphi \in C_{C}^{\infty}(G) \\
\|\varphi\|_{\infty} \leq 1}}\left\|(I-L(k)) A^{\beta}\left(\lambda I+\overline{H_{g}^{P}}\right)^{-1} A^{\alpha_{2}}\left(\lambda I+\overline{H_{g}^{P}}\right)^{-1} \cdots A^{a} l\left(\lambda I+\overline{H_{g}^{P}}\right)^{-1} \check{\varphi}\right\|_{\infty} \\
& \leqq \sup _{g \in F^{\prime}}\left\|(I-L(k)) A^{\beta}\left(\lambda I+\overline{H_{g}^{P}}\right)^{-(m-l+1-\varepsilon) / m}\right\|_{\infty \rightarrow \infty} \cdot\left\|A^{\left(\alpha_{2}^{\prime}\right) *}\left(\lambda I+\left(H_{g}^{P}\right)^{*}\right)^{-(l-1+\varepsilon) / m}\right\|_{1 \rightarrow 1} \\
& \left.\cdot \prod_{j=2}^{l-1}\left\|A^{\alpha_{j}^{\prime \prime}}\left(\lambda I+\overline{H_{g}^{P}}\right)^{-(m-l+j-1+2-1) / m}\right\|_{\infty \rightarrow \infty} \cdot \prod_{j=3}^{l} \| A^{\left(\alpha_{j}^{\prime}\right) *}\left(\lambda I+H_{g}^{P *}\right)^{*}\right)^{-(l-j+1+2-1) / m} \|_{1 \rightarrow 1} \\
& \cdot \| A^{a_{l}^{\prime \prime}}\left(\lambda I+{\overline{H_{g}^{P}}}^{-1} \|_{\infty \rightarrow \infty}\right. \text {. }
\end{aligned}
$$

But

$$
\left\|A^{\alpha}\left(\lambda I+\overline{H_{g}^{P}}\right)^{-a}\right\|_{\infty \rightarrow \infty} \leqq c \lambda^{-(m a-|\alpha|)}
$$


for all $|\alpha|<m a \leqq m$ and all $\lambda$ large enough, for some constant $c>0$ independent of $g$. A similar estimate is valid on the dual space. Moreover,

$$
\begin{aligned}
& \left\|(I-L(k)) A^{\beta}\left(\lambda I+\overline{H_{g}^{P}}\right)^{-(m-l+1-\varepsilon) / m}\right\|_{\infty \rightarrow \infty} \\
& \quad=\sup _{\substack{\varphi \in L_{\infty} \\
\|\varphi\|_{\infty} \leq 1}}\left\|(I-L(k)) A^{\beta}\left(\lambda I+\overline{H_{g}^{P}}\right)^{-(m-l+1-\varepsilon) / m} \varphi\right\|_{\infty} \\
& \quad \leqq c\left(|k|^{\prime}\right)^{r} \sup _{\substack{\varphi \in L_{\infty} \\
\|\varphi\|_{\infty} \leqq 1}}\left\|\left(\lambda I+\overline{H_{g}^{P}}\right)^{-(m-l+1-\varepsilon) / m} \varphi\right\|_{\infty ;|\beta| ; \gamma, \infty} \\
& \quad \leqq c\left(|k|^{\prime}\right)^{r} \sup _{\substack{\varphi \in L_{\infty} \\
\|\varphi\|_{\infty}}} \Gamma(m-l+1-\varepsilon)^{-1} \int_{0}^{\infty} d t t^{m-l+1-\varepsilon-1} e^{-\lambda t}\left\|S_{t}^{g} \varphi\right\|_{\infty ;|\beta| ; \gamma, \infty} \\
& \quad \leqq c c^{\prime}\left(|k|^{\prime}\right)^{r} \Gamma(m-l+1-\varepsilon)^{-1} \int_{0}^{\infty} d t t^{m-l+1-\varepsilon-1} e^{-\lambda t} t^{-(|\beta|+\gamma) / m} \\
& \quad \leqq c^{\prime \prime}\left(|k|^{\prime}\right)^{r} \lambda^{(-m+l-1+\varepsilon+|\beta|+\gamma) / m}
\end{aligned}
$$

for some $c>0$ which depends only on $\gamma$ and $m$, and some $c^{\prime}>0$, which is uniform for $g \in G$ because it is obtained from interpolation of the bounds on $\left\|S_{t}^{g}\right\|_{L_{\infty} \rightarrow L_{\infty}}$ and $\|\left[S_{t}^{g} \|_{L_{\infty} \rightarrow L_{\infty ; n}^{\prime}}\right.$. Combining these estimates we obtain the bounds

$$
\left\|(I-L(k)) A^{\alpha} r_{\lambda} \varphi\right\|_{\infty} \leqq c\left(|k|^{\prime}\right)^{\gamma} \lambda^{-(m-|\alpha|-\gamma) / m}\|\varphi\|_{\infty}
$$

for all $k \in B_{1}^{\prime}$ and $\varphi \in L_{\infty}$.

Step 2. Let $c_{\alpha} \in L_{\infty ; m}^{\prime}$ for all $\alpha \in J\left(d^{\prime}\right)$ with $|\alpha|=m$.

In this step we remove the restriction of the $C^{\infty}$-differentiability of the coefficients. Let $H_{0}, H_{1}, r_{\lambda}^{(n)}, r_{\lambda}$ and $Q_{\lambda}$ be as in the proof of Theorem 4.1. Let $\alpha \in J_{m-1}\left(d^{\prime}\right)$ and $k \in B_{1}^{\prime}$. By Step 1 there exists $c>0$, independent of $n$ and $\lambda$ such that

and

$$
\left\|(I-L(k)) A^{\alpha} r_{\lambda}^{(n)} \varphi\right\|_{\infty} \leqq c\left(|k|^{\prime}\right)^{\gamma} \lambda^{-(m-|\alpha|-\gamma) / m}\|\varphi\|_{\infty}
$$

$$
\left\|A^{\alpha} r_{\lambda}^{(n)} \varphi\right\|_{\infty} \leqq c \lambda^{-(m-|a|) / m}\|\varphi\|_{\infty}
$$

for all $\varphi \in L_{\infty}, n \in \boldsymbol{N}$ and large $\lambda>0$. Now let $\varphi \in L_{\infty}$. Then for all $\phi \in L_{1 ; m-1}^{\prime}$ and $\alpha \in J_{m-1}\left(d^{\prime}\right)$ we have

$$
\left|\left(A^{\alpha} \psi, r_{\lambda} \varphi\right)\right|=\lim _{n \rightarrow \infty}\left|\left(A^{\alpha} \psi, r_{\lambda}^{(n)} \varphi\right)\right| \leqq c \lambda^{-(m-|\alpha|) / m}\|\phi\|_{1}\|\varphi\|_{\infty}
$$

for all large $\lambda>0$. So $r_{\lambda} \varphi \in L_{\infty ; m-1}^{\prime}$. Moreover, for all $k \in B_{1}^{\prime}$ and $\varphi \in C_{c}^{\infty}(G)$ one has

$$
\begin{aligned}
\left|\left(A^{a}(I-L(k)) \psi, r_{\lambda} \varphi\right)\right| & =\lim _{n \rightarrow \infty}\left|\left(A^{\alpha}(I-L(k)) \phi, r_{\lambda}^{(n)} \varphi\right)\right| \\
& \leqq c\left(|k|^{\prime}\right)^{r} \lambda^{-(m-|\alpha|-\gamma) / m}\|\|_{1}\|\varphi\|_{\infty}
\end{aligned}
$$

for all large $\lambda>0$. So

Hence

$$
\left\|(I-L(k)) A^{\alpha} r_{\lambda} \varphi\right\|_{\infty} \leqq c\left(|k|^{\prime}\right)^{\gamma} \lambda^{-(m-|a|-r) / m}\|\varphi\|_{\infty} .
$$




$$
\left\|(I-L(k)) A^{\alpha}\left(\lambda I+\bar{H}_{0}\right)^{-1} \varphi\right\|_{\infty} \leqq c\left(|k|^{\prime}\right)^{\gamma} \lambda^{-(m-|a|-\gamma) / m}\|\varphi\|_{\infty}
$$

since $\left(\lambda I+\bar{H}_{0}\right) r_{\lambda}=I+Q_{\lambda}$ and $Q_{\lambda}$ is a bounded operator with norm less than $2^{-1}$ for large $\lambda$.

Now we return to the operator $H$. By (38) the operator $H_{1}$ is a small perturbation of the operator $\bar{H}_{0}$, so by the proof of Theorem 3.5 in [6] we have that $H_{1}\left(\lambda I+\bar{H}_{0}\right)^{-1}$ is a bounded operator with norm less than $2^{-1}$ for large $\lambda$ and

$$
(\lambda I+\bar{H})^{-1}=\left(\lambda I+\bar{H}_{0}\right)^{-1}\left(I-H_{1}\left(\lambda I+\bar{H}_{0}\right)^{-1}\right)^{-1}
$$

for all large $\lambda$. So $(\lambda I+\bar{H})^{-1} \varphi \in L_{\infty ; m-1}^{\prime}$ and

$$
\left\|(I-L(k)) A^{a}(\lambda I+\bar{H})^{-1} \varphi\right\|_{\infty} \leqq c\left(|k|^{\prime}\right)^{r} \lambda^{-(m-|a|-r) / m}\|\varphi\|_{\infty}
$$

for all $\alpha \in J_{m-1}\left(d^{\prime}\right), k \in B_{1}^{\prime}$ and large $\lambda$. This proves (40) and

$$
\left\|(\lambda I+\bar{H})^{-1} \varphi\right\|_{\infty ; m-1 ; r, \infty ; K \leqq} \leqq \lambda^{-(m-|a|-r) / m}\|\varphi\|_{\infty} .
$$

Step 3. Suppose $c_{\alpha} \in L_{\infty}^{\prime} m$ for all $\alpha \in J\left(d^{\prime}\right)$ with $|\alpha|=m$ and $q \in[1, \infty]$. It follows by interpolation from (41) that

$$
\left\|(\lambda I+\bar{H})^{-1} \varphi\right\|_{\infty ; m-1 ; \gamma, q ; K \leqq} \leqq c \lambda^{-(m-|a|-\gamma) / m}\|\varphi\|_{\infty}
$$

for all large $\lambda, \varphi \in L_{\infty}, x \in J_{m-1}\left(d^{\prime}\right)$ and $\gamma \in\langle 0,1\rangle$. Hence $D(\bar{H}) \subseteq\left(L_{\infty ; m-1}^{\prime}\right.$, $\left.L_{\infty ; m}^{\prime}\right)_{r, q ; K}$ and

for all $\varepsilon>0$.

$$
\|\varphi\|_{\infty ; m-\imath ; \gamma, q ; K \leqq} \leqq \varepsilon^{1-\gamma / m}\|\bar{H} \varphi\|_{\infty}+c \varepsilon^{-(m-1+\gamma) / m}\|\varphi\|_{\infty}
$$

Finally note that since $S$ is holomorphic on each of the $L_{p}$-spaces it follows that $S_{t} L_{p} \subseteq D(\bar{H})$ for all $t>0$. Therefore $S_{t} L_{p} \subseteq\left(L_{\infty ; m-1}^{\prime}, L_{\infty ; m}^{\prime}\right)_{r, q ; K}$. A similar conclusion is valid for $L_{\hat{p}}$.

If one applies the foregoing arguments to the subcoercive operator $H$ acting on $C_{0}$ instead of on the $L_{p}$-spaces then one draws an analogous conclusion

$$
D(\bar{H}) \subseteq\left(C_{0 ; m-1}^{\prime}, C_{0 ; m}^{\prime}\right)_{r, q ; K} \text {. }
$$

But $S_{t} L_{p} \leqq C_{0}$ for $p \in[1, \infty\rangle$ as a consequence of the 'Gaussian' bounds on the semigroup kernel. Hence $S_{t} L_{p} \subseteq S_{t / 2} C_{0} \subseteq D(\bar{H})$. Thus we can improve the last statement of Theorem 2.1.

Corollary 4.4. Let $H$ be an $m$-th order subcoercive operator with coefficients $c_{\alpha} \in L_{\infty ; m}^{\prime}$ for all $\alpha \in J\left(d^{\prime}\right)$ with $|\alpha|=m$ and $S$ the corresponding interpolating semigroup. Then

$$
S_{t} L_{p} \subseteq\left(C_{0 ; m-1}^{\prime}, C_{0 ; m}^{\prime}\right)_{r, q ; K}, \quad S_{t} L_{\hat{p}} \subseteq\left(C_{0 ; m-1}^{\prime}, C_{0 ; m}^{\prime}\right)_{\gamma, q ; K}
$$

for all $t>0, p \in[1, \infty\rangle, \gamma \in\langle 0,1\rangle$ and $q \in[1, \infty]$. 
The estimates (40) also give bounds on 'Lipschitz derivatives' of $L_{\infty}$-functions and the semigroup kernel.

Corollary 4.5. Let $H$ be an m-th order subcoercive operator acting on $L_{\infty}$ with coefficients $c_{\alpha} \in L_{\infty ; m}^{\prime}$ for all $\alpha \in J\left(d^{\prime}\right)$ with $|\alpha|=m$. Then for $\gamma \in\langle 0,1\rangle$ there exist $c>0$ such that

$$
\left\|(I-L(k)) A^{\alpha} \varphi\right\|_{\infty} \leqq\left(|k|^{\prime}\right)^{r}\left(\varepsilon^{1-(|a|+\gamma) / m}\|\bar{H} \varphi\|_{\infty}+c \varepsilon^{-(|\alpha|+r) / m}\|\varphi\|_{\infty}\right)
$$

for all $k \in B_{1}^{\prime}, \alpha \in J_{m-1}\left(d^{\prime}\right), \varphi \in D(\bar{H})$ and $\varepsilon \in\langle 0,1]$. Consequently the corresponding semigroup kernel $K$ satisfies bounds

$$
\mid\left((I-L(k)) A^{\alpha} K_{t}\right)(g ; h) i \leqq c\left(|k|^{\prime}\right)^{\gamma} t^{-\left(D^{\prime}+|\alpha|+\gamma\right) / m} e^{\omega t}
$$

for all $k \in B_{1}^{\prime}, \alpha \in J_{m-1}\left(d^{\prime}\right), t>0$ and uniformly for all $g, h \in G$.

Proof. The $L_{\infty}$-inequalities are a reexpression of the estimates (40) with $\varepsilon=\lambda^{-1}$. The kernel bounds for small $t$ then follow from the observation that

$$
\begin{aligned}
\left|\left((I-L(k)) A^{\alpha} K_{t}\right)(g ; h)\right| & \leqq \mid(I-L(k)) A^{\alpha} S_{t} \|_{\hat{1} \rightarrow \infty} \\
& \leqq\left(|k|^{\prime}\right)^{\gamma}\left(\varepsilon^{1-(|\alpha|-\gamma) / m}\left\|\bar{H} S_{t}\right\|_{\hat{1} \rightarrow \infty}+c \varepsilon^{-\left(\mid \alpha_{1}+\gamma\right) / m}\left\|S_{t}\right\|_{\hat{1} \rightarrow \infty}\right)
\end{aligned}
$$

together with the kernel bounds of Theorem 4.1 and the choice $\varepsilon=t$. The bounds for large $t$ follow by use of the semigroup property from the small $t$ bounds.

\section{§. Conclusion}

Although the foregoing results provide a fairly satisfactory basis for the theory of subcoercive operators with variable coefficients they do leave open a number of obvious questions concerning regularity properties. First, we have shown that the semigroup kernels associated with $m$-th order operators with smooth coefficients are themselves smooth but we have only obtained "Gaussian" bounds on derivatives of order less than or equal to $m-1$. Naturally one expects such bounds in general. Secondly, we have established that if the principal coefficients of the $m$-th order operator are $m$-times diflerentiable then the range of the corresponding semigroup $S$ consists of functions which are "almost" $m$ times differentiable. But if the lower order coefficients are also sufficiently differentiable then one might expect the range of $S$ to consist of functions which are more than $m$-times differentiable, i. e., one would expect the action of $S$ to be smoother than the principal coefficients. Properties of the latter type have been established in the special case of second-order operators with real coefficients in [4]. Thirdly, it would be ideal if one could completely relate smoothness of the coefficients and the action of the semigroup. For example, if the leading coefficients are $n$-times differentiable and the lower order ones $(n-m)$ - 
times, with $n>m$, does $S$ map into functions which are 'almost' $(n+m)$-times differentiable? In this respect it is worth noting that Theorem 3.6 of [1] gives conditions under which the semigroup $S$ associated with a strongly elliptic second-order operator maps into the $(n+1)$-times differentiable functions in $L_{2}$.

We conclude with some remarks on the simplest and most significant case of second-order operators. In particular we compare and contrast our results in this case with the complementary results obtained earlier in [4]. Note that each second-order operator $H$ can be expressed in the form

$$
H=-\sum_{i, j=1}^{d^{\prime}} c_{\imath \jmath}-A_{\imath} 1_{\jmath}+\sum_{i=1}^{d^{\prime}} c_{\imath} A_{\imath}+c_{0}
$$

and subcoercivity corresponds to uniform strict positive-definiteness of the real part of the matrix $C=\left(c_{\imath \jmath}\right)$ of principal coefficients, i. e., there is a $\mu>0$, the ellipticity constant, such that $2^{-1}\left(C+C^{*}\right) \geqq \mu I$, in the sense of matrices, uniformly over the group (see [10] Proposition 6.7).

The principal result, Theorem 1.1, of [4] imposes the two additional restrictions

a. the $c_{\imath \jmath}$ are real-valued and $c_{\imath \jmath}=c_{\jmath \iota}$,

b. the $c_{\imath \jmath} \in L_{\infty ; 1}^{\prime}$,

and concludes that

1. $H$ has a family of closed extensions $\widetilde{H}$ on the $L_{p^{-}}$, and $L_{\hat{p}^{-}}$, spaces which generate a consistent interpolating semigroup $S$,

2. $S$ is holomorphic on each of the spaces $L_{p}$ or $L_{\hat{p}}$,

3. the range of each $S_{\iota}, t>0$, consists of functions which are 'almost' twice left-differentiable in the subelliptic directions.

By the last statement we mean that the functions are once left-differentiable in the subelliptic directions and the derivatives are Lipschitz continuous with exponent, measured relative to the subelliptic directions, arbitrarily close to one.

In contrast Theorem 4.1, with $m=2$, states that if

$\mathrm{a}^{\prime}$. the $c_{\imath \jmath}$ are complex-valued,

$\mathrm{b}^{\prime}$. the $c_{\imath \jmath} \in L_{\infty ; 2}^{\prime}$, then

$1^{\prime}$. the closures of $H$ generate a consistent interpolating semigroup $S$,

$2^{\prime}$. if $\nu I \geqq(2 i)^{-1}\left(C-C^{*}\right) \geqq-\nu I$ and $\theta_{C}=\arctan \mu / \nu$ then $S$ is holomorphic on each $L_{p^{-}}$, and $L_{\hat{p}^{-}}$, space in a sector with angle $\theta \geqq \theta_{C}$,

$3^{\prime}$. the range of each $S_{t}, t>0$, consists of functions which are 'almost' twice left-differentiable in the subelliptic directions.

Thus our current assumption $a^{\prime}$. is less restrictive than the assumption a. of $[4]$ but $b^{\prime}$. is more restrictive than $b$. The current conclusions $1^{\prime}$. and $2^{\prime}$. are, however, stronger than the comparable conclusions 1 . and 2. of [4] whilst 3 . and $3^{\prime}$. are identical. It is remarkable that under the assumptions a. and b. of $[4]$ the action of the semigroup $S$ is smoother than the principal coefficients 
and it is this phenomenon of increased smoothing that needs to be better understood.

There are also several other similar implications contained in [4] and the foregoing sections. For example, the identification $\widetilde{H}=\bar{H}$ is established on $L_{\infty}$ in [4] for real-valued $c_{\imath j} \in L_{\infty ; 3}^{\prime}$ but much greater smoothness is required both of the principal coefficients and the lower order coefficients to obtain a similar result on the $L_{p}$-spaces with $p<\infty$, and in this respect Theorem 4.1 is a distinct improvement. In addition both Theorem 1.1 of [4] and Theorem 4.1 give existence of a semigroup kernel with Gaussian bounds etc.

It is possible to improve both the above results by combination of the techniques used in their proofs. The strong points of the [4] result are based on approximation arguments, similar to those used in Section 4, which involve a priori inequalities for subelliptic operators with smooth real-valued coefficients. There are three types of a priori inequality used, the $L_{2^{-}}$, and $L_{\infty}$, bounds of Proposition 3.1 and 3.2 of [4] and the Gaussian bounds on the semigroup kernel in Proposition 10.1 of [4]. The important feature of all these bounds is they only involve first derivatives of the $c_{\imath}$ in the subelliptic directions. But the $L_{2}$-bounds can easily be established for second-order operators with complex coefficients by repetition of the arguments of [4]. Positive-definiteness of the matrix $\left(c_{\imath j}\right)$ is replaced by positive-definiteness of its real part. The proof of appropriate $L_{\infty}$-bounds is slightly more delicate. The proof in [4] relies on some basic $L_{\hat{p}}$-bounds for operators with constant coefficients given by Rothschild and Stein [21] and repetition of the arguments of [4] requires an extension of the Rothschild-Stein bounds to complex-valued operators. In fact the RothschildStein proof of their inequalities does not require reality of the operators but also applies to operators for which the matrix of principal coefficients is hermitian. But the general case is a consequence of Folland's inequalities, [12], Theorem 6.1, for stratified groups and the Rothschild-Stein lifting technique. Finally the derivation of kernel bounds which are only weakly dependent on the smoothness of the coefficients can be achieved by modification of the derivation given in [4] for the real-valued operators. This argument is based on the adaptation by Fabes and Stroock [11] of a technique of Davies [7]. The Davies method uses $L_{2}$-positivity techniques on the $L_{p}$-spaces with $p \geqq 2$ combined with Nash inequalities to bound the norm of $S$ as an operator from $L_{\hat{2}}$ to $L_{\infty}$. This approach can be adapted to operators with complex coefficients and this will be discussed in a later paper.

\section{References}

[1] Arendt, W., Batty, C. J.K. and Robinson, D. W., Positive semigroups generated by elliptic operators on Lie groups, J. Operator Theory. 23 (1990), 369-407.

[2] Bratteli, O. and Robinson, D.W., Operator algebras and quantum statistical mechanics, 1, Second edition, Springer, New York etc., 1987. 
[3」 Bratteli, O. and Robinson, D.W., Second-order elliptic operators and heat kernels on Lie groups, Trans. Amer. Math. Soc., 325 (1991), 683-713.

[4] - Subelliptic operators on Lie groups : variable coefficients, Acta Applicandae Mathematicae (to appear).

[5] Butzer, P.L. and Berens, H., Semi-groups of operators and approximation, Die Grundlehren der mathematischen Wissenschaften 145, Springer-Verlag, Berlin etc., 1967.

[6] Davies, E. B., One-parameter semigroups, London Math. Soc. Monographs 15, Academic Press, London etc., 1980.

$[7\rfloor-$, Heat kernels and spectral theory, Cambridge Tracts in Mathematics 92, Cambridge University Press, Cambridge etc., 1989.

[8] Elst, A.F.M. ter, On infinitely differentiable and Gevery vectors for representations, Proc. Amer. Math. Soc., 112 (1991), 795-802.

[9] Elst, A.F.M. ter and Robinson, D. W., Subelliptic operators on Lie groups: regularity, Journal of the Australian Mathematical Society (1994). To appear.

[10] - Subcoercivity and subelliptic operators on Lie groups, I, II. J. Pot. Anal. (to appear).

L11」 Fabes, E. B. and Stroock, D. W., A new proof of Moser's parabolic Harnack inequality using the old ideas of Nash, Arch. Rat. Mech. and Anal., 96 (1986), 327338.

[12」 Folland, G.B., Subelliptic estimates and function spaces on nilpotent Lie groups, Arkiv för matematil, 13 (1975), 161-207.

[13] Hebisch, W., Sharp pointwise estimate for the kernels of the semigroup generated by sums of even powers of vector fields on homogeneous groups, Stud. Math., 95 (1989), 93-106.

[14] Hebisch, W., Estimates on the semigroups generated by left invariant operators on Lie groups, J. Reine Angeu'. Math., 423 (1992), 1-45.

[15] Helffer, B., Partial differential equations on nilpotent groups. In Herb, R., Johnson, R., Lipsman, R., and Rosenberg, J., eds., Lie group rcpresentations, III, Lecture Notes in Mathematics 1077, Springer-Verlag, Berlin etc., 1984, 210-253.

[16] Hochschild, G., The structure of Lie groups, Holden-Day, San Francisco etc., 1965.

[17] Nagel, A.. Stein, E. M. and Wainger, S., Balls and metrics defined by vector fields I: basic properties, Acta Math.. 155 (1985), 103-147.

L18」 Norris, J.R. and Stroock, D. W., Estimates on the fundamental solution to heat flows with uniformly elliptic coefficients, Proc. Lond. Math. Soc., 62 (1991), 373-402.

[19] Ouhabaz, E.-M., $L^{\infty}$-contractivity of semigroups generated by sectorial forms, Tubingen Somesterbericht Funktionalanalysis, 19 (1990/1991), 159-174.

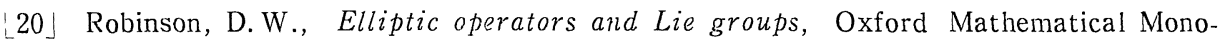
graphs, Oxford University Press, Oxford etc., 1991.

[21] Rothschild, L. P. and Stein, E. M., Hypoelliptic differential operators and nilpotent groups, Acta Math., 137 (1976), 247-320.

[22] Saloff-Coste, L. and Stroock, D. W., Operateurs uniformement sous-elliptiques sur les groupes de Lie, J. Funct. Anal., 98 (1991), 97-121.

[23] Stroock, D. W., Estimates on the heat kernel for second order divergence form operators, Lecture notes, 1990. 
